\title{
ATM phosphorylates PP2A subunit A resulting in nuclear export and spatiotemporal regulation of the DNA damage response
}

Amrita Sule ${ }^{1,2}$, Sarah E. Golding ${ }^{1}$, Syed F. Farhan ${ }^{1,2}$, James Watson ${ }^{1}$, Mostafa H. Ahmed $^{3}$, Glen E. Kellogg ${ }^{3,7}$, Tytus Bernas ${ }^{4}$, Sean Koebley ${ }^{5}$, Jason C. Reed ${ }^{5,7}$, Lawrence F. Povirk ${ }^{6,7}$, and Kristoffer Valerie ${ }^{1,2,7,{ }^{*}}$

Departments of ${ }^{1}$ Radiation Oncology, ${ }^{2}$ Biochemistry and Molecular Biology, ${ }^{3}$ Medicinal Chemistry, ${ }^{4}$ Anatomy, ${ }^{5}$ Physics, ${ }^{6}$ Pharmacology and Toxicology, and the ${ }^{7}$ Massey Cancer Center, Virginia Commonwealth University, Richmond, VA 23298, USA

Keywords: CRM1, DNA repair, mitotic catastrophe, NES, nuclear export, phosphatase, PP2A shuttling, radiation

Running title: ATM-PP2A signaling in the DDR

${ }^{*}$ Correspondence to:

Kristoffer Valerie, Ph.D.

Department of Radiation Oncology

Virginia Commonwealth University

Richmond, VA 23298-0058

Phone: 804 628-1004

FAX: 804 828-6042

Email: kristoffer.valerie@vcuhealth.org

Supported in part by NIH R01NS064593 and R21CA194789 (K.V.). 


\section{Abstract}

Ataxia telangiectasia mutated (ATM) is a serine-threonine protein kinase and important regulator of the DNA damage response (DDR). One critical ATM target is the structural subunit A (PR65) of protein phosphatase 2A (PP2A), known to regulate diverse cellular processes such as mitosis and cell growth as well as dephosphorylating many proteins during the recovery from the DDR. We generated mouse embryonic fibroblasts expressing PR65-WT, -S401A (cannot be phosphorylated), and -S401D (phosphomimetic) transgenes. Significantly, S401 mutants exhibited extensive chromosomal aberrations, impaired DNA double-strand break (DSB) repair and underwent increased mitotic catastrophe after radiation. Our study demonstrates that the phosphorylation of a single, critical PR65 amino acid (S401) by ATM fundamentally controls the DDR, and balances DSB repair quality, cell survival and growth by spatiotemporal PR65 nuclearcytoplasmic shuttling mediated by the nuclear export receptor CRM1. 


\section{Introduction}

Protein phosphatase 2A (PP2A) is a major serine/threonine (S/T) phosphatase that regulates many cellular processes including DNA replication, cell cycle progression, cell differentiation, and the DNA damage response (DDR) (1-3). The mammalian PP2A holoenzyme is a hetero-trimer ( $A B C)$ comprised of a scaffolding subunit ( $A$ or PR65), a regulatory subunit $(B)$, and a catalytic subunit $(C)$, or a heterodimer (AC; PP2A Core enzyme) (4). Both the $A$ and $C$ subunits have each two isoforms that are evolutionary conserved (5,6). The two PR65 isoforms, A $\alpha$ (PPP2R1A) and A $\beta$ (PPP2R1B) are 86\% identical. Each PR65 isoform is shaped into a horseshoe composed of 15 non-identical internal repeats of a 39 amino acid sequence, termed HEAT (ㅂuntingtin, Ellongation factor 3, Protein phosphatase 2A-subunit $\underline{A}$, and the yeast kinase IOR1) motif (7-9). Almost $90 \%$ of the PP2A holoenzymes comprise of the $A \alpha$ isoform whereas $A \beta$ is primarily associated with development and the CNS (10). There are at least 17 subtypes of the B subunit, classified into four distinct families, B/PR55, B'/PR61, B"'/PR72 and PTP/PR53 $(4,6,11,12)$. Specific regulatory B subunits control the diversity and target specificity of the various PP2A holoenzymes. The association of the AC heterodimer with a specific B subunit imparts complete activity, sub-cellular localization, and substrate specificity to the heterotrimeric holoenzyme.

Ataxia telangiectasia mutated (ATM), an S/T kinase, is defective in the human autosomal recessive disorder ataxia-telangiectasia (A-T) and is one of the major regulators of the cellular response to DNA damage (13). ATM is activated by DNA double-strand breaks (DSBs) induced endogenously by growth factor signaling, and 
exogenously by DNA damaging agents like ionizing radiation (IR) and radiomimetic drugs. In the event of DNA damage, ATM and other PIKKs such as A-T and RAD3related (ATR), and DNA-PKcs get activated and direct a coordinated response referred to as the DDR by acting at several levels, including cell cycle checkpoints, DNA repair, and apoptosis (13-18). In a sense, ATM acts as a cellular rheostat balancing and coordinating the DDR with cell growth to maintain homeostasis (19). DDR proteins, phosphorylated in response to DNA damage are subsequently dephosphorylated by phosphatases to reverse the DDR and reset the cell to normalcy $(14,20)$. We have previously reported that the inhibition of ATM kinase blocks pro-survival signaling in human tumor cell lines by reducing AKT (S473) phosphorylation (21). This study revealed that ATM regulates AKT phosphorylation via an okadaic acid (OA) sensitive phosphatase. OA inhibits the S/T protein phosphatases protein phosphatase 1 (PP1) and PP2A, exhibiting greater affinity and inhibition towards the latter (22-24).

Protein phosphatase $2 \mathrm{~A}$ regulates key DDR proteins ATM, ATR, CHK1, CHK2, and p53 (25-28), controls the G2/M checkpoint (29), and facilitates DSB repair via the dephosphorylation of $\gamma-\mathrm{H} 2 \mathrm{AX}$ in response to IR (30). More specifically, PP2A binds to ATM and regulates ATM (S1981) auto-phosphorylation and activation in an indirect manner (25). Furthermore, it was reported that PP2A is regulated by ATM-mediated phosphorylation of PR65 at S401 resulting in the retention of histone deacetylase HDAC4 in the cytoplasm (31). Consequently, without functional ATM HDAC4 is unable to shuttle between the cytoplasm and nucleus where it permanently remains resulting in neurodegeneration which is a characteristic hallmark of A-T. Even though this study did not address the impact of PR65 S401 (de)phosphorylation during the DDR, a major 
conclusion from the report was that PP2A is negatively regulated by ATM, which does fit well with how we perceive ATM to regulate PP2A and AKT phosphorylation (21).

In the present study we generated PR65 depleted mouse embryonic fibroblasts (MEFs) expressing PR65 site-specific S401 mutants; a S401A mutant which cannot be phosphorylated as well as a phospho-mimetic S401D mutant. We found that these mutant cells were impaired in the DDR, displayed increased radio- and chemosensitivity, and had fundamentally altered DSB repair. Both S401 mutants were deficient in HRR with S401D also being severely compromised in non-homologous end joining (NHEJ) whereas S401A appeared to possess aberrant, greater than normal end joining activity. Upon assessing further, the phenotype of these cells, we uncovered a completely rewired DSB repair machinery due to PP2A malfunctioning. Here we report that the spatial and temporal regulation of PR65 phosphorylation at S401 by ATM through cytoplasmic-nuclear shuttling is critical for regulating DSB repair quality and the proliferative recovery from the DDR. 


\section{MATERIAL AND METHODS}

Plasmids. Previously described pMIG-Aalpha WT retroviral vector with a Flag-tag at the $\mathrm{NH}_{2}$ terminal (32) obtained from Addgene (plasmid \#10884) was generously provided by William Hahn. Mutations at the S401 codon and other positions were generated using QuikChange site-directed mutagenesis (Stratagene). The PR65 mutations were verified by DNA sequencing. Other plasmids used were Flag-hCRM1, a gift from Xin Wang (Addgene plasmid \# 17647): pmCherry-C1-RanQ69L, a gift from Jay Brenman (Addgene plasmid \# 30309 ): pCSCMV:tdTomato, a gift from Gerhart Ryffel (Addgene plasmid \# 30530): human TERT (LOX-TERT-iresTK; Addgene plasmid \#12245), generously provided by Didier Trono; DR-GFP plasmid (33); and pcDNA3-H2BmCherry (Addgene plasmid \#20972) (34,35). PR65 was cloned into pcDNA3-mRuby2 (Addgene plasmid \#40260), generously provided by Michael Lin, into pGEX-2T (GE Life Sciences \#28954653) and pPAmCherry-a-tubulin (Addgene plasmid \#31930) provided by Vladislav Verkhusha. Mutations at the S401 and L373 positions were generated using QuikChange site-directed mutagenesis (Stratagene). The PR65 mutations were verified by DNA sequencing.

Antibodies and other reagents. Antibodies used for western blotting and/or immunocytochemistry were anti-ATM (Cat \#2873), anti-AKT (Cat \#2920), anti-p(S473) AKT (Cat \#9271), anti-PR65 (Cat \#2039), anti-PP2A-C (Cat \#2038), anti-pS139-( $\gamma)$ H2AX (Cat \#9718) and anti-GST (Cat \#2624) purchased from Cell Signaling Technology. Anti- $\gamma-H 2 A X$ (Cat \#05-636), anti-GAPDH (Cat \#MAB374) were purchased from Millipore Sigma. Anti-53BP1 (Cat \#NP100-304), anti-Rad51 (Cat \#NB100-148), 
and anti-PyMT (Cat \#NB100274955) were purchased from Novus Biologicals. Antip(S4/S8)-RPA (Bethyl laboratories, Cat \#A300-245A), anti-ERK2 (Cat \#sc-154), antipERK1/2 (Cat \#sc-7383) and anti-CRM1 (Cat \#sc-74454) were purchased from Santa Cruz Biotechnology, and anti-Flag (Sigma Aldrich, Cat \#F1365). Alexa Fluor 594 goatanti-mouse immunoglobulin $\mathrm{G}(\lg G)$, Alexa Fluor 680 goat-anti-rabbit $\lg G$, and Alexa Fluor 680 goat anti-mouse IgG (Life Technologies), Dylight 800 anti-mouse IgG (Cat \#5257) and Dylight 800 anti-rabbit IgG (Cat \#5151) were from Cell Signaling Technology. Leptomycin B (Cat \#L2913) and camptothecin (Cat \#7689-03-4) were from Sigma Aldrich. KU-60019 was from Selleck (Cat \#S1570).

\section{Generation of PR65 conditional knockout mouse embryonic fibroblasts.}

All animal breeding and experiments were approved by Virginia Commonwealth University IACUC. Fvb.129S-PPP2R1A $A^{\text {tm } 1 \text { witrj }}$ mice (36) were obtained from Jackson laboratories as stock number 017441 . These CKO mice possess loxP sites flanking exons 5-6 of the PR65 gene (PPPP2R1A). Heterozygous 129S-PPP2R1A tm1wltrj (CKO/WT) mice were bred to obtain 129S-PPP2R1A $A^{\text {tm1w/tr/tm1w/tr }}(\mathrm{CKO} / \mathrm{CKO})$ mice. Embryos were harvested at $13.5 \mathrm{dpc}$ from a pregnant CKO/CKO female mouse and PR65-CKO MEFs generated by the VCU Massey Cancer Center Transgenic/Knockout Mouse Core using standard methods (37).

Cell culture and irradiation. HEK293, U1242, U87 glioma cells, and MEFs were cultured in DMEM (GIBCO) medium supplemented with 10\% FBS and 1\% Pen-Strep (Life Technologies). Serum starvation was for 16 hours (wherever mentioned) in 
medium without FBS. Irradiations were done using an MDS Nordion Gammacell-40 research irradiator with $137-\mathrm{Cs}$ source delivering a dose rate of $1.05 \mathrm{~Gy} / \mathrm{min}$. Cell growth was determined by CellTiter-Glo® Luminescent Cell Viability Assay (Promega, Cat \#G7570). Cells were serially diluted and seeded in a 96-well plate. At days 2, 5 and 7 after seeding, CellTiter-Glo® reagent was added to the medium at the recommended final concentration. Plates were incubated for 10 minutes at room temperature and luminescence determined using an EnVision Multilabel reader (PerkinElmer) and the readings taken as the measure of cell growth. Clonogenic survival assays were carried out as described $(38,39)$.

Microscopy and cell imaging. For DNA repair foci assay, cells were grown on LabTek (Naperville, IL) glass slides. After treatment, cells were fixed with $3 \%$ paraformaldehyde, made permeable with $0.5 \%$ Triton-X-100 in phosphate buffered saline (PBS) and blocked with casein (ThermoFisher Scientific Cat \#37528). Cells were stained with primary antibodies diluted in casein blocker followed by secondary antibodies, and nuclei were counterstained with DAPI $(1 \mu \mathrm{g} / \mathrm{ml})$ and mounted in Vectashield $®$ mounting medium (Vector Labs). Cells were imaged and analyzed using Zeiss LSM 710 imaging system in the VCU Microscopy Facility.

For photoactivation studies to capture representative images, HEK293 cells were transfected with pPAmCherry-PR65 (WT, S401A and S401D) in glass bottom dishes and $48 \mathrm{~h}$ post-transfection sub-nuclear ROI $(4 \mu \mathrm{m} \times 4 \mu \mathrm{m})$, marked by the squares, were photoactivated by $405 \mathrm{~nm}$ laser $(242.8 \mu \mathrm{J})$ and followed over time $(<300 \mathrm{~s})$ by live cell imaging using a Zeiss LSM 710 microscope with images acquired at $63 x$. For 
quantitative photoactivation studies, HEK293 cells, stably transfected with linearized pPAmCherry-PR65 (WT, S401A and S401D, NES, S401A-NES and S401D-NES), were cultured under G418 (500 $\mu \mathrm{g} / \mathrm{ml})$ selection in a glass bottom dish for $48 \mathrm{~h}$. Next, the cells were imaged with Zeiss LSM 880 confocal system, based on Axio Z1 inverted stage and equipped with 40x PlanApo oil immersion objective (NA = 1.4), 405 nm diode laser (30 mW) and $561 \mathrm{~nm}$ DPSS laser (20 mW). Fluorescence of mCherry was excited with the latter laser attenuated to $0.8 \%$ and detected in $570-620 \mathrm{~nm}$ range with a multi-anode (spectral) GaAsP hybrid detector in the integrative mode at 950V gain. Time series of transmitted light and fluorescence images were collected with $0.28 \mu \mathrm{m}$ pixel size, $3.07 \mu$ s dwell time (236 $\mu$ s frame time) and $256 \times 256$ pixel frame size. Following first 25 frames $2.8 \times 2.8 \mu \mathrm{m}$ regions in cell nuclei were irradiated with $325.2 \mu \mathrm{J}$ of $405 \mathrm{~nm}$ light. Next, imaging mCherry fluorescence resumed for $277 \mathrm{~s}$ (1175 frames). Up to 7 irradiation regions were selected in each field of view using transmitted light image as the reference.

In vitro kinase assay. YFP-ATM was immunoprecipitated from stably transfected HEK293 cells by GFP-TRAP (Chromotek). The immunoprecipitates were suspended in kinase assay buffer containing $2 \mu \mathrm{Ci}$ of $\left[\gamma^{32}{ }^{32}\right]$ ATP, $20 \mu \mathrm{M}$ unlabeled ATP, and GSTsubstrates (GST-PR65 or GST-p53). GST-PR65 has GST fused to full-length PR65 and GST-p53 expresses the first 100 amino acids of p53 (40). GST-fusions were purified from extracts of BL21 (DE3)/pLysS cells using Glutathione Sepharose 4B beads as recommended by the manufacturer (GE Healthcare Life Sciences). The kinase reaction was conducted at $30^{\circ} \mathrm{C}$ for 20 minutes and stopped by adding $2 \mathrm{X}$ Laemmli loading 
buffer. Samples were separated on $10 \%$ polyacrylamide gels, followed by drying of the gel onto 3M paper, exposed to a screen, scanned by a Phosphorimager (Typhoon) and the signal quantified (40).

Transfection. Superfect, Effectene (Qiagen) or PEI were used to transfect cells transiently and for stable drug selection. Electroporation of MEFs was done using an Amaxa Nucleofector apparatus. Sterile Solution I: $20 \%$ ATP-disodium salt, $12 \% \mathrm{MgCl}_{2}$ (Filter Sterilize, adjust $\mathrm{pH}$ to 7.4 ) and Solution II: $2 \% \mathrm{KH}_{2} \mathrm{PO}_{4}, 2 \% \mathrm{NaHCO}_{3}, 0.06 \%$ glucose ( $\mathrm{pH}$ to 7.4 ) were combined 1:50 (total volume $-100 \mu \mathrm{l}$ ) prior to electroporation (41). Electroporation was done with program: N-024.

DNA repair assays. pEGFP-C1 (Clontech) plasmid was digested with BamHI and Bglll to generate a 51-bp fragment which was inserted into the BamHI site of pCSCMV:tdTomato to generate a Smal restriction site immediately downstream from the BamHI site. The modified pCSCMV:tdTomato + linker plasmid was linearized with Smal followed by BamHI to create incompatible DNA ends and gel purified prior to use. pCSCMV:tdTomato+linker plasmid $(1 \mu \mathrm{g})$ was transfected into the MEFs (from a $80 \%$ confluent 6 -cm plate) and cells divided equally into the wells of a 12-well plate. Cells were collected at 2, 4 and 8 hours from the media and pooled with the remaining cells after trypsinization. A small fraction was used to determine RFP+ cell numbers using a Nexcelom cellometer (Nexcelom Bioscience). Remaining cell pellets were re-suspended in 15\% sucrose, $50 \mathrm{mM}$ Tris- $\mathrm{HCl}, 25 \mathrm{mM}$ EDTA buffer supplemented with $0.1 \%$ TritonX100 and treated with RNase. Cell lysates were incubated at $50^{\circ} \mathrm{C}$ for $3-4$ hours. Equal 
amount of re-suspension buffer was combined with equal volume of $0.1 \%$ SDS and 50 $\mu \mathrm{g} / \mathrm{ml}$ of Proteinase K. Samples were incubated at $60^{\circ} \mathrm{C}$ overnight. This was followed by phenol chloroform treatment and DNA was precipitated with 1:1 isopropanol. The level of repaired DNA was assessed by quantitative real-time PCR, using an ABI 7900HT (Applied Biosystems) and SYBR green detection. The (Forward 5' CGGAGCAAGCTTGATTTAGGTG 3' and Reverse 5’ CGCATGAACTCTTTGATGACCTC 3') primers were used to analyze the repair junction between BamHI and Smal with primers for ampicillin gene (Forward 5'TGTGCAAAAAAGCGGTTAGCT 3' and Reverse 5'GCGGCCAACTTACTTCTGACA 3') were used for template normalization.

MEFs were arrested in mitosis by treating cells with $50 \mathrm{ng} / \mathrm{ml}$ of nocodazole for 16 hours. Cells were collected for western blotting at 1,2 and 4 hours after nocodazole washout with p-PLK1 (T210). MEFs were treated with $1 \mathrm{mM}$ of hydroxyurea (HU) for 2 hours followed by a washout of the drug and cells collected at 0,1 , and 6 hours after washout and western blotting with antibodies specific for pRPA (S4/S8) and $\gamma-\mathrm{H} 2 \mathrm{AX}$.

In vitro end joining assay was carried out at $37^{\circ} \mathrm{C}$ in $50 \mathrm{mM}$ triethanolammonium-

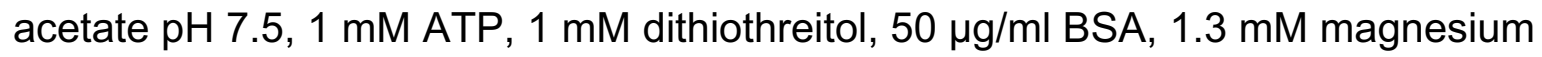
acetate and dNTPs at $100 \mu \mathrm{M}$ each. Typically, a 16- $\mu$ l reaction contained $10 \mu \mathrm{l}$ of whole-cell extract (44), resulting in a final concentration of $8 \mathrm{mg} / \mathrm{ml}$ protein, $66 \mathrm{mM}$ potassium acetate and $16 \%$ glycerol, and an effective $\mathrm{Mg}^{2+}$ concentration of $1 \mathrm{mM}$. The substrate was an internally labeled plasmid with partially cohesive ends, prepared as described $(42,43)$. Homologous recombination GFP assay (DR-GFP) was carried out as previously described by determining GFP+ cells by flow cytometry $(44,45)$. PR65 flox/flox 
MEFs were stably transfected with linearized DR-GFP plasmid and selection with puromycin followed by infection with WT, S401A or S401D pMIG-A $\alpha$ (PR65) lentivirus. Pooled GFP+ cells were then infected with Ad-Cre to remove the endogenous PR65 (see Fig. 1).

CRISPR-Cas9 DSB repair and chromosomal translocation assay was performed as described $(46,47)$. PR65-KO MEFs (WT, S401A and S401D) were infected with lentivirus expressing Cas9 and gRNA (Rosa26) and (Rosa26; H3f3b), respectively, by introducing one or two guide RNAs (5'-GTTGGCTCGCCGGATACGGG-3' for H3f3b; 5'ACTCCAGTCTTTCTAGAAGA-3' for Rosa26) into LentiCRISPR v2 (Addgene plasmid \#52961) and LentiCRISPRv2 hygro (Addgene Plasmid \#98291). Plasmids were sequenced to confirm the correct gRNA insertions. To generate mixes of stable cell populations lentivirus was made in HEK293T cells and MEFs infected followed by selection with puromycin or hygromycin as described $(48,49)$. The Rosa26 and H3f3b primers used for PCR were those described (47). Amplified products were cloned in pGEM®-T Easy (Promega) and sequenced to verify translocation and junction sequences.

Chromosome translocation and amplicon length heterogeneity were quantified using high-speed atomic microscopy (HSAFM) with sample preparation and execution of the technique described in detail previously (50-52). Briefly, a tip with a $<10 \mathrm{~nm}$ sharpened point was rastered across a surface, and the height of the tip measured to yield a threedimensional map of the sample features with nanoscale resolution (53). Our high-speed atomic force microscope (Bristol Nanodynamics) routinely acquires $2 \mu \mathrm{m} \times 2 \mu \mathrm{m}$ images with single-nanometer resolution at a rate of 1 image per second (54). To deposit DNA 
onto an atomically flat surface for imaging, we first purified the DNA from PCR reactions using Ampure (Beckman Coulter), then added $\mathrm{MgCl}_{2}$ to facilitate adhesion of DNA to negatively-charged mica. The resulting DNA solution was drop-deposited onto freshly cleaved mica (Ted Pella, Inc), dried with compressed air, and baked for 10 minutes at $120^{\circ} \mathrm{C}$. Following HSAFM imaging, we employed a custom line-by-line processing program to flatten the images, which is required to account for mechanical and thermal vibration. To quantify DNA lengths observed in the flattened images, we used custom computer vision programming to automate DNA tracing and filter out strands that did not meet certain quality metrics, e.g. strands that were branched or circular. Analysis of traced length distributions was performed by fitting Gaussian mixture models, then analyzing the Bayesian information criteria to determine the number of Gaussian distributions that produced the best fit. The $95 \%$ confidence interval for the mean of each fitted Gaussian was determined using a bootstrapping approach with $n=10,000$. All programming and analyses were conducted using MATLAB.

Molecular modeling studies. Molecular Dynamics (MD) simulations were carried out with the NAMD 2.8 package developed by the theoretical and computational biophysics group in the Beckman institute for Advanced Science and Technology at the University of Illinois at Urbana-Champaign (55). CHARMM (Charmm-27) was used as the force field (56). The initial MD setup was done using VMD (57). Both S401 phosphorylated and unphosphorylated PR65 3D models were solvated in an equilibrated TIP3P water box. Then $\mathrm{Cl}^{-}$and $\mathrm{Na}^{+}$ions were added to neutralize the system and appropriate number of ions added up to a concentration of $50 \mathrm{mM}$. Solvent molecules were first 
minimized for 500 steps of conjugate gradients minimization method, keeping the protein molecules fixed to allow favorable distribution of water molecules on the complex surface. Subsequently, the system was coupled to a heat bath from 0 to $300^{\circ} \mathrm{K}$ and the constraints applied to the solute atoms were gradually decreased after which, the system was allowed to be simulated without restraints for over a period of 10 ps. Finally, a 50 ns molecular dynamics production phase was carried out on the entire system. The analysis of the MD trajectory was done in VMD (56). HINT analysis was carried out on published x-ray crystal structures of the Holo and Apo PR65 protein (PDB IDs 2NPP and 1B3U respectively), which were also used for the docking studies with the CRM1 protein (PDB ID 3GB8). Protein-protein docking was performed using the HADDOCK (High Ambiguity Driven DOCKing) algorithm (58). The residues from CRM1 forming the protein-protein binding interface with Snurportin-1 were used as constraints for docking together with the identified putative leucine-rich nuclear export signal on PR65 $(59,60)$. Docking results were individually inspected after which high scoring models were passed into the refinement step. All docked poses were refined with the FireDock (Fast Interaction Refinement in a molecular Docking) algorithm (61), rescored and ranked using the HINT force field that describes and quantifies all interactions in the biological environment by exploiting the interaction information implicit in $\log \mathrm{P}_{\mathrm{o} / \mathrm{w}}$ (the partition coefficient for 1-octanol/water solute transfer) $(62,63)$. HINT is known as a "natural" force field because it is based on empirical energetic terms that are defined by real experiments, and thus encodes interaction types including Coulombic, hydrogen bond and hydrophobic interactions expected between molecules in the biological 
environment as a free energy force field that includes solvation/desolvation and entropy in addition to the other enthalpic terms.

Statistics. Unpaired two-tailed $t$ tests or one way ANOVA were performed on triplicate or more data sets using GraphPad Prism 3.0 (Graphpad Software, inc.). $P$-values are indicated as ${ }^{*},<0.05 ;{ }^{* *},<0.01$; and ${ }^{* * *},<0.001$. 


\section{RESULTS}

PP2A functions downstream of ATM to regulate AKT. In a previous study from our laboratory, we demonstrated that ATM indirectly regulates the phosphorylation of AKT at S473 via an okadaic acid (OA) sensitive phosphatase (21). It is known that several oncogenic DNA viruses such as SV40 and Polyoma interfere with PP2A activity in infected cells with SV40 small t-antigen and PyMT blocking PP2A activity by replacing the B subunit (64-66) (Supplemental Fig.1A). After examining the effect of an ATM kinase inhibitor (ATMi) in a panel of tumor cell lines including human U87 and U1242 glioma cells as well as HEK293 and HEK293T carcinoma cells, inhibition of AKT phosphorylation was observed across the board relative to untreated cells with the exception of HEK293T cells (Supplemental Fig.1B). HEK293T expresses SV40 t/T antigens, which inversely correlates with the lack of PAKT inhibition by ATMi, suggesting similar or overlapping mechanism of action. To build on this finding, we infected U87 and U1242 cells with retroviruses expressing PyMT or not (Supplemental

Fig. 1C, D). ATMi did not appear to reduce the levels of PAKT in glioma cells overexpressing PyMT. This effect was independent of exposure to ionizing radiation (IR) $(21,67)$. The presence of the PyMT gene in these cell populations was verified by PCR (Supplemental Fig. 1E). We confirmed the ability of OA to increase the levels of phosphorylated AKT (21), this time in a nuclear extract with nM concentrations suggesting that PP2A is acting on AKT in the nucleus (Supplemental Fig. 1F), perhaps as a complex in direct physical association with AKT (68). Altogether, these results suggest that ATM regulates the phosphorylation of AKT indirectly via the inhibition of PP2A activity. 
Generation of PR65 KO MEFs complemented with S401 mutant alleles. Ruediger et.al. demonstrated that PR65 is an essential gene, since knocking out PR65 resulted in embryonic lethality (36). We generated homozygous PR65 conditional knockout (CKO) mouse embryonic fibroblasts (MEFs) after breeding heterozygous 129S-PPP2R1A $A^{\text {tm } 1 \text { witr/j }}$ mice to obtain 129S-PPP2R1A $A^{\text {tm1wltr/m1w/tr }}(\mathrm{CKO} / \mathrm{CKO})$ mice. Floxing out PR65 exon 5 and 6 generates PR65 KO MEFs (Fig. 1A). MEFs generated from 129S-

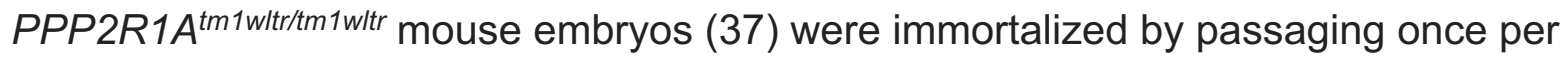
week and infecting the cells with a lentivirus expressing human TERT. We generated two site-specific mutations at the S401 codon of PR65 using the retroviral vector pMIGAalpha WT as template (32): a S401A mutant that cannot be phosphorylated and a S401D phospho-mimetic mutant. The MEFs were then separately infected with the retroviruses expressing Flag - tagged PR65 WT, S401A or a S401D mutant in addition to GFP (via an IRES). The endogenous PR65 alleles were then floxed out by infecting the cells with an adenovirus expressing Cre recombinase (Ad-Cre). Complete floxing was confirmed by PCR (Fig. 1B). MEFs that were not complemented with virus expressing PR65 did not survive after infection with Ad-Cre (Fig. 1C), in line with that PR65 is essential in the mouse (36). Pooled MEFs from each infection were shown to be close to $100 \%$ GFP+ (Fig. 1D). Western blotting with anti-PR65 and -Flag antibodies showed that at the protein level all endogenous PR65 (Fig. 1E, left panel; lower band), was replaced with the slightly larger Flag-PR65 protein expressed from the retrovirus. Altogether, we generated a panel of isogenic WT, S401A, and S401D cell populations expressing PR65 at approximately the same levels as endogenous PR65 (compare band intensity of PR65 doublet (Fig. 1E, right panel; lane 1). 
ATM kinase phosphorylates PR65 in vitro. The PR65 S401 residue is part of an S/Q- ATM kinase signature motif highly conserved in PR65's among mammals including human and mouse and is a bona fide substrate for ATM phosphorylation (31). To obtain an abundant source of co-expressed ATM and PR65 for further analysis and experimentation, we first attempted to isolate stably transfected HEK293 cells coexpressing mRuby2-PR65 and YFP-ATM after selection with G418 (expressed from YFP-ATM) and isolating cells expressing both YFP and Ruby. However, we were only able to recover YFP-ATM/S401A cells (Supplemental Fig. 2A), suggesting that overexpression of ATM with either S401D or WT was not tolerated.

To determine whether ATM phosphorylates PR65 in vitro and to confirm previously documented results (31), YFP-ATM was immunoprecipitated from HEK293 cells expressing YFP-ATM only (Supplemental Fig. 2B) and utilized for in vitro kinase assays with $\left[\gamma_{-}{ }^{32} \mathrm{P}\right]$ ATP and GST-PR65FL as substrate. The result shows that ATM kinase phosphorylates PR65 in vitro and that phosphorylation is significantly reduced in the presence of an ATMi (Supplemental Fig. 2C, compare lanes 5 and 6). Similarly, GST-p531-100, a known substrate of ATM kinase at p53 S15 (40), was also phosphorylated and inhibited in the presence of the ATMi. This result confirms that ATM kinase is able to phosphorylate PR65 in vitro in line with a previous report (31).

\section{A phospho-mimetic (S401D) mutant prevents the association with ATM, PP2A} catalytic subunit and AKT. Goodarzi et al. showed that PP2A exerts its control over ATM by interacting via the PR65 subunit in a manner lost in irradiated cells (25). To determine whether the S401D amino acid replacement affected the interaction with 
ATM, we immunoprecipitated over-expressed Flag-PR65 WT, -S401A and -S401D, respectively, from transduced HEK293 cells. Flag-PR65 IP's were then analyzed for the presence of endogenous ATM, the catalytic subunit of PP2A (PP2A-C), and AKT. We found an association of ATM, PP2A-C, and AKT with the WT and the nonphosphorylatable S401A but not with S401D (Fig. 2A). The lack of an interaction between S401D and ATM but not WT or S401A suggests that the PP2A Core enzyme (AC) is possibly in a complex with ATM, in agreement with previous work (25). That the S401D phospho-mimetic disrupts this complex is new information and fits well with the dissociation of ATM and PP2A complex after irradiation and the phosphorylation of PR65 S401 by ATM $(25,31)$. That AKT is found complexed with PR65 is no surprise (68), but that S401D disrupts this interaction is intriguing, even though we cannot tell from this result whether ATM and AKT are in the same or separate PR65 complexes.

To support a critical role for S401 phosphorylation by ATM in the disruption of such physical interaction we performed molecular modeling studies based on the known PR65 crystal structure (PDB ID 2IAE). Two 50 ns Molecular Dynamics runs were carried out with phosphorylated S401 PR65 as well as the unphosphorylated structure (Fig. 2B, C). The result suggests that phosphorylation at S401 induces a local movement of the $\alpha$-helices on either side of the helix (residues 375-454) encompassing S401 ultimately causing a conformational change of the entire PR65 protein, particularly at its $\mathrm{N}$-terminal portion. This conformational change results in the distortion of the horseshoe shape of the subunit by $\sim 4.5 \AA$ compared to the unphosphorylated structure (15.321-10.873). Altogether, this modeling suggests that phosphorylation at S401 could 
cause the dissociation of the physical interaction with its binding partners ATM and AKT.

Growth and radiation signaling are altered in $\mathbf{S 4 0 1}$ mutant cells. We then examined whether AKT signaling and growth was affected in response to insulin stimulation and radiation (21). WT, S401D, and S401A MEFs were serum-starved prior to stimulation with insulin followed by western blotting for pAKT (S473) (Fig. 3). We found that S401D cells responded more robustly to insulin when PAKT levels were determined over the course of 60 minutes (Fig. 3A), which correlated with an elevated growth rate of S401D relative to WT and S401A cells (Fig. 3B). Similar to AKT signaling, ERK signaling is associated with pro-survival and increased cell growth after low radiation doses, which in part is regulated by ATM $(45,69)$. When we examined ERK signaling in unstimulated cells we observed elevated pERK1/2 levels in S401A and more so in S401D cells compared with WT, suggesting that AKT and ERK signaling are positively affected when S401 is mutated, with S401D cells being affected the most (Fig. 3C). Furthermore, after a single radiation dose of 2 Gy, S401 mutant cells showed dampened responses when analyzed for both $\gamma-\mathrm{H} 2 \mathrm{AX}$ and pAKT by western blotting (Fig. 3D). It is well-established that ATM phosphorylates $H 2 A X$ at $S 139(\gamma-H 2 A X)$ in response to radiation at the sites of DSBs (70). Furthermore, PP2A modulates DSB repair via the dephosphorylation of $\gamma-\mathrm{H} 2 \mathrm{AX}$ to reverse chromatin accessibility (30). On examining the $\gamma-\mathrm{H} 2 \mathrm{AX}$ levels in more detail, we found that in WT cells the levels of $\gamma$ H2AX peaked at 15-30 min post irradiation and then declined. However, $\mathrm{H} 2 \mathrm{AX}$ phosphorylation was reduced in both S401A and S401D cells with the latter trending 
even higher than WT and S401A at 60 minutes (Fig. 3D). Similarly, pAKT levels increased after IR in WT and less so in S401A and S401D cells. These results suggest that after low dose radiation, S401 mutant cells have dampened pAKT and $\gamma-\mathrm{H} 2 \mathrm{AX}$ levels likely as a result of malfunctioning DDR. Additionally, S401D seems to overcome this initial blockade with a delayed response and increased $\gamma-\mathrm{H} 2 \mathrm{AX}$ levels at later times. Altogether, we conclude that $\$ 401$ mutant cells have aberrant responses to insulin and radiation yet distinct from each other, resulting in altered DDR signaling and proliferative activity compared to WT cells.

\section{PR65 mutants have impaired G2/M checkpoint resulting in aberrant mitosis and}

elevated levels of mitotic catastrophe. PP2A plays distinct roles at different stages of mitosis while associated with different $B$ subunits $(71,72)$. PP2A regulates mitotic entry and exit, as well as playing a role in the protection of centromeres by inhibiting PLK1 and Aurora A kinases (73-77). Taking into account the importance of PP2A in mitosis at multiple steps and elsewhere during the cell cycle, we examined whether S401 mutant cells show any perturbations in mitosis and cell cycle checkpoints. We found that the mitotic index (cells undergoing mitosis/total number of cells) was reduced in WT cells after radiation but not in S401 mutant cells, suggesting that the radiation-induced G2/M check point is intact in WT cells, as expected, but not functioning properly in the mutants (Fig. 4A, B). Furthermore, S401 mutants had significantly increased numbers of basal and radiation-induced (2-3 fold) chromosomal aberrations (Fig. 4C). To support this finding, live cell imaging of MEFs expressing a histone H2B-mCherry reporter revealed that the S401 mutant cells exhibited abnormal mitoses including the formation 
of micronuclei and elevated levels of mitotic catastrophe, and the formation of tetraploid cells whose frequency increased after exposure to radiation (Fig. 4D, left panel). Again, S401D cells showed more aberrations than S401A. Furthermore, in undamaged cells, the length of mitosis in S401 mutant cells was significantly longer than in WT cells (Fig. 4D, right panel). To examine mitosis in more detail, we then focused on exit from mitosis. PLK1 is known to be critical during G2/M entry as well as for successful chromosome separation and exit from mitosis (78). Normally, pPLK1 (T210) is elevated in mitosis and upon exit is dephosphorylated by PP2A (79). A nocodazole blockade synchronized the cell panel in mitosis and following washout, we collected cells for western blotting with anti-pPLK1 (T210) antibody (Fig. 4E). Mitotic S401 mutant cells showed elevated pPLK1 levels compared with WT at 1 hour and exited mitosis faster than WT cells with S401D showing the most pronounced effect (Fig. 4F, G). Together, both mitotic entry and exit are abnormal in S401 mutant cells reflected in significantly longer mitosis and clear signs of struggle to get through mitosis.

To get better insight into what occurs during S-phase we then examined the recovery of collapsed replication forks in the cell panel by determining pRPA32 (S4/8) and $\gamma$ H2AX levels after the release from a hydroxyurea (HU) blockade (Fig. 4H). A previous study showed that PP2A is critical for the dephosphorylation of pRPA32, cell cycle reentry and efficient DSB repair presumably by HRR (80). We found that S401 mutant cells had reduced $\gamma-\mathrm{H} 2 \mathrm{AX}$ levels after $\mathrm{HU}$ release, which peaked at $\sim 1$ hour post washout (Fig. 4H; bottom left panel). Perhaps more importantly, S401D cells showed a delay in the removal of pRPA32 (Fig. 4H; bottom right panel) indicative of an alteration in PP2A activity during fork recovery. This result suggests that the recovery of collapsed 
replication forks is impaired in S401 mutant cells and that PP2A, at least in S401D cells, might adversely affect pRPA32 dephosphorylation and perhaps subsequently impact HRR. In addition, a colony-forming radiosurvival experiment demonstrated that S401D cells were also significantly more radiosensitive than WT cells, with S401A trending more radiosensitive (Fig. $\mathbf{4 I}$ ). The same pattern of sensitivity was seen when the cell panel was exposed to camptothecin (CPT), a topoisomerase I inhibitor, known to produce DSBs preferentially in S and G2 (Supplemental Fig. 3).

Altogether, increased chromosomal aberrations seen in untreated S401 mutant cells (and more so after radiation) are suggestive of impaired G2/M checkpoint and aberrant mitosis (entry, duration, and exit), resulting in elevated levels of mitotic catastrophe and increased sensitivity to DNA damage. In addition, replication fork recovery and DSB repair were impaired, especially in the S401D cells. It was however unexpected to see that the impact on survival was relatively minor considering the major negative effects on the G2/M checkpoint and increased mitotic struggle seen in S401 mutant cells.

S401 mutants have aberrant DSB repair. In addition to reversing changes in chromatin structure caused by $\mathrm{H} 2 \mathrm{AX}$ phosphorylation, PP2A is believed to facilitate NHEJ by dephosphorylating KU70/80 and DNA-PKcs, which are critical participants in NHEJ (14), and to promote their release from DNA (81). Furthermore, PP2A restores kinase activity of phosphorylated, inactive DNA-PKcs in vitro to regulate NHEJ (82). To determine how S401 alterations might affect DSB repair we then carried out in vivo as well as in vitro end joining assays. First, in a reporter assay, WT, S401A and S401D mutant cells were transfected with a linearized pCSCMV:tdTomato plasmid with 
incompatible restriction endonuclease DNA ends unable to ligate. Once the plasmid is repaired and circularized, the red fluorescence protein (RFP) expressed is a measure of end joining activity (Fig. 5A). Post transfection, cells were collected at various times and RFP+ cells quantified. At 16 hours the S401A cells had almost twice the number of RFP+ cells relative to WT cells whereas the S401D cells had much less than the WT cells throughout (Fig. 5B, top panel). To examine early repair, we quantified the circularized plasmid at 1 and 4 hours after transfection and determined end joining yield by quantitative PCR (Fig. 5B, bottom panel). The PCR result is in agreement with what we observed with the reporter assay, i.e., S401D cells have compromised end joining. S401A cells probably did not have enough time to translate into elevated repair over WT seen at 16 hours.

To assess the possible effect of PP2A-mediated dephosphorylation on end joining in vitro, a ${ }^{32} \mathrm{P}$-oligonucleotide ligated into a plasmid substrate bearing partially complementary $5^{\prime}$ overhangs was incubated in whole-cell extracts from WT, S401A, and S401D cells for 1.5 or 6 hours (Fig. 5C). As in our previous work $(43,83)$, the predominant 34-base head-to-tail product as well as the 16-base head-to-head product reflects $3^{\prime}$ resection and annealing at a 4-base micro-homology (Fig. 5C, left panel). We observed dramatically reduced end joining activity with the extract from S401D, whereas the S401A extract markedly enhanced it and yielded an additional 42-base product corresponding to gap filling without resection (Fig. 5C, middle and right panels). Thus, while the critical PP2A dephosphorylation targets are currently unknown these results show that using S401 mutants as surrogates for mimicking (de)phosphorylated S401 is important for end joining by reporter as well as in cell-free in vitro assays. In line 
with these results, S401D cells showed abnormal, extensive and more persistent 53BP1 foci after radiation compared with WT (Fig. 5D). 53BP1 is critical for directing DSB repair towards NHEJ at the expense of HRR (84). 53BP1 foci co-localized with $\gamma-\mathrm{H} 2 \mathrm{AX}$, suggesting that NHEJ is significantly altered and delayed in S401D cells. Furthermore, S401A cells had fewer but more pronounced foci at 5 hours than either WT or S401D

(Fig. 5D). Altogether, S401 mutant cells have significantly altered end joining activity in quality as well as temporal execution yet S401D and S401A differed in that the latter showed super-active end joining whereas S401D was severely compromised.

S401 mutants are HRR impaired. Since NHEJ and HRR are in competition in S/G2 of the cell cycle and we observed irregular end joining in the S401 mutants, we then examined HRR in the cell panel. The PP2A holoenzyme associated with the B55 subunit is implicated in HRR via the modulation of ATM phosphorylation and the G1/S checkpoint (85), as well as the dephosphorylation of pRPA32 mediated by PP2A after replication fork recovery, which might impact HRR (80). To determine the importance of the S401 residue in HRR, we generated CKO/CKO MEFs with the DR-GFP repair reporter (44) followed by infection with either WT, S401A and S401D retrovirus and floxing of the endogenous PR65 gene with Ad-Cre (Supplemental Fig. 4) as before (see Fig. 1B, E). We saw that HRR was significant reduced in S401A and S401D cells represented by lower GFP+ events (Fig. 5E). During HRR, single stranded DNA generated during DNA resection is first coated with RPA and then replaced by RAD51 during Holliday junction formation and resolution (14). This step is essential for BRCA1 recruitment and efficient HRR (86). Therefore, we treated the cells with CPT and then 
co-stained for pRPA32 (S4/8) and RAD51. Both S401A and S401D cells had 40\% less RAD51 and pRPA32 foci compared with WT cells (Fig. 5F). This finding supports the result with the DR-GFP reporter and the conclusion that HRR is impaired in both S401A and S401D cells relative to WT. Altogether, it is quite clear that S401 mutant cells have rewired DDR and show aberrant DSB repair. Surprisingly, however, these mutants display fairly minor effects on cell survival after radiation and CPT treatment. This is particularly striking with the S401D cells since they are compromised in end joining as well as HRR.

\section{Increased aberrant chromosomal DSB repair and translocation in $\mathbf{5 4 0 1}$ mutants.}

Alternative end joining (Alt-EJ, now referred to as Theta-mediated end joining; TMEJ) and other micro-homology repair pathways are believed to become engaged when cNHEJ (classical end joining) and/or HRR are not fully functional or being disengaged $(46,47,87)$. However, TMEJ is highly mutagenic because of the promiscuous activity imposed by DNA polymerase Theta $(\mathrm{Pol} \theta)$ - mediated chromosomal repair and rearrangements through short (2-4 bp) stretches of micro-homology in resected DNA ends. To assess TMEJ in our PR65 cell panel we examined DSB repair quality after CRISPR/Cas9-mediated DNA cleavage at the Rosa26 locus and by chromosomal translocation between the Rosa26 and H3fl3 loci on Chr. 6 and 11, respectively $(46,47)$. The fact that the Rosa26 cleavage site is positioned in the middle of an Xbal site allows for the elimination of any DNA resealing products without indels, which if present would destroy the Xbal site, as well as DNA that was not cut (Fig. 6). As expected, Xbal- 
resistant PCR fragments remained after cleavage with Cas9-Rosa26/H3f3b gRNAs expressed but not without (Fig. 6A).

Because of the complexity of analysis and difficulties achieving meaningful statistical support for differences in the quality and quantity of end joining products between cells in the panel, we decided to examine the spectrum of translocations occurring between Chr. 6 and 11 after inducing dual chromosomal breaks $(46,47)$. TMEJ appears to play an important role in chromosomal translocations in human cells (88-91). However, in the mouse, the role of Pol Theta in translocation repair is more controversial (92). Nevertheless, with PCR primers amplifying translocation events as an expected 415-bp fragment we found that the S401D cells produced relatively large insertions as well as deletions compared with either WT or S401A cells which gave bands closer to the expected PCR fragment size without large indels (Fig. 6B, left panel). The PCR fragments were cloned and some sequenced (Supplemental Fig. 5). Among the DNA sequences, we found indels with micro-homology in the DNA from S401D and S401A cells. Altogether, translocations generated in S401D cells are to a large extent associated with significant micro-homology end joining since predominant number of DNA sequences had short 2-4 bp repeats in the junction between Chr. 6 and 11. In line, with the results generated throughout this study using different DNA repair assays, we again noticed differences in the quality of DSB repair between the three cell populations.

Assessing translocation events by atomic force microscopy. Because cloning of PCR fragments might be biased against isolating longer inserts many of which might 
have direct or inverted repeats (that could be unstable in bacteria), we decided to utilize a novel, high-throughput technique that does not require plasmid cloning. A recently developed alternative for determining translocations by indel size is by using atomic force microscopy (AFM) (51). High-speed AFM-based physical mapping of DNA allows single-molecule measurement of thousands of PCR products in a relatively short time. However, only the size of the indels are determined without establishing the DNA sequence but the assay is nevertheless a way to quantify differences in DNA repair quality between the three cell populations, simplify the process, and, importantly, allow for better statistical analysis. In this experiment, we noticed that S401A as well as S401D gave translocation indels seen on an agarose gel whereas few were seen from WT cells or a synthetic (Syn) template without any indel at the Rosa26-H3f3b junction that served as standard (Fig. 6C, panel a). On the other hand, when we examined the AFM results using the same PCR reactions as examined by agarose electrophoresis we noticed shifts in the size of indels between the three cell populations (Fig. 6C, panels be) and images of the DNA corresponding to the various curves (Fig. 6C, panels f-i). The Syn template produced sizes centered at $119 \mathrm{~nm}$ (blue; 93\%) and a bulge of smaller sizes around $45 \mathrm{~nm}$ (orange; 6\%) representing background noise products (e, i, Supplemental Table 1). WT cells predominantly gave sizes around $113 \mathrm{~nm}$ (blue; $81 \%$ ) slightly smaller than with the Syn standard template, indicating smaller deletions at the junction as expected as well as smaller fragments around $48 \mathrm{~nm}$ (orange; 15\%) some of which possibly represent deletions of 200 -bp or more $(\mathbf{b}, \mathbf{f})$. The size pattern resulting from S401A was more complex with a main peak at $108 \mathrm{~nm}$ (blue; 56\%), a broad peak centered at $143 \mathrm{~nm}$ and extending $>260 \mathrm{~nm}$ (purple; 20\%) in size, and a range of 
deletions with peaks between 49-61 nm (green; 12\%, orange; 11\%). Finally, S401D produced a predominant peak centered around $106 \mathrm{~nm}$ (blue; 61\%), slightly smaller in size then either WT or S401A and an abundant peak centered around $47 \mathrm{~nm}$ (orange; 34\%). The overall pattern and difference in fragment size distribution of indels seen between WT, S401A, and S401D by AFM are significant (Supplemental Table 1). The observed distinct peaks might represent repair events resulting from preferred microhomology hotspots such as repetitive DNA in resected ends that might serve as sinks for repair. Clearly, the quality of DSB repair is dramatically altered when S401 is replaced with either a null or a phospho-mimetic substitution reflecting the importance of PR65 S401 (de)phosphorylation during the DDR. However, to determine the mechanism behind these differences and whether it represents TMEJ or other (backup) DSB repair goes beyond the scope of this report and will have to wait for a more thorough future investigation.

\section{S401 (de)phosphorylation shuttles PR65 between the nucleus and the cytoplasm.}

To return to the overarching goal of this study and how growth, cell cycle regulation, and DSB repair could be so fundamentally altered by a single amino acid change mimicking the phosphorylation or not of PR65 at S401 by the ATM kinase, we wanted to explore this issue more thoroughly. In neurons, ATM phosphorylates PR65 at S401 resulting in nuclear-cytoplasmic shuttling of HDAC4 and PR65 (31). Thus, we examined the cellular location of WT, S401A and S401D PR65 in the MEFs using Flag antibody. In WT cells PR65 was present in the cytoplasm as well as the nuclear compartment whereas S401A was predominantly nuclear and in S401D exclusively cytoplasmic with little 
change after radiation except that in the WT and S401A cells PR65 appeared more in

punctate nuclear foci (Fig. 7). After irradiation of WT cells, PR65 seemed to translocate to the cytoplasm even though it is unlikely that all PR65 complexes participate in a unison manner blurring the response and interpretation thereof. This observation in addition to the sequestration of S401D mutant in the cytoplasm suggests that the phosphorylation of PR65 at S401 causes it to translocate from the nucleus to the cytoplasm in line with a previous report (31). To explore the mechanism, we treated the cells with leptomycin B (LMB), which is a very specific inhibitor of nuclear export protein CRM1 (chromosome region maintenance 1) responsible for the nuclear export of many proteins and RNAs (93). After LMB treatment of WT cells, PR65 began to accumulate in the nucleus and combined with irradiation this effect was even more pronounced (Fig. 7B, compare middle panels at $30 \mathrm{~min}+/-$ LMB). In support of this finding, S401A cells did not respond to radiation with PR65 remaining in the nucleus whereas in S401D cells PR65 was almost exclusively cytoplasmic regardless of being irradiated or not (Fig.

7A). These results suggest that nuclear export of PR65 after radiation and phosphorylation by ATM kinase is mediated by CRM1 (Fig. 7C).

\section{PR65 shuttles to the cytoplasm by CRM1 via a nuclear export sequence. After}

DNA damage several DDR proteins including p53, BRCA1, and BRCA2 are exported out of the nucleus through NES-mediated CRM1 processes (94-96). To determine whether PR65 might have a NES, we scanned the PR65 amino acid sequence for putative hydrophobic leucine residue motifs using the online tool NetNES1.1 (http://www.cbs.dtu.dk/services/NetNES/). We identified two leucine residues, L370 and 
L373, that scored positive as potential residues of a NES sequence between PR65 amino acids 365-373 (LLPLFL370AQL 373 ) which resembles the consensus NES LxxxLxxLxL (97) (Supplemental Fig. 6A). Nuclear export of proteins harboring a functional NES sequence requires CRM1 binding to the target (98-100). We therefore examined whether CRM1 and PR65 interact by performing co-immunoprecipitation of lysates of HEK293 cells expressing Flag-CRM1 and mRuby2-PR65 also including a plasmid expressing the RanQ69L mutant that is incapable of exchanging GTP for GDP and prevent the dissociation of the Ran-CRM1 complex in whole cell extracts $(101,102)$. The immunoprecipitation/western blotting experiment shows that CRM1 interacts with PR65 (Fig. 8A). One prototype CRM1 interactive partner is Snurportin-1 (Exportin-1) for which a co-crystal structure has been determined (PDB ID 3GB8) This structure was then used for identifying possible CRM1-PR65 hydrophobic interactions by HINT analysis modeling hydrophobic protein interactions under aqueous condition (103-105).

We found that the docked CRM1-PR65 model has a HINT score more than 4-fold higher than CRM1-Snurportin-1 that is reduced when WT is replaced with S401A and increased with S401D. In addition, the CRM1-PR65 interface area was significantly larger compared with CRM1-Snurportin-1 (Fig. 8B). Furthermore, modeling suggests an interaction between CRM1-K537 and the $-\mathrm{L}_{365} \mathrm{LPLFLAQL}_{373}-$ motif of PR65 with S401 positioned in the vicinity of the binding interface (Fig. 8C). Upon S401 phosphorylation, a tighter interaction is expected between K537 and pS401, or alternatively S401D, because of increased electrostatic interaction (Supplemental Fig. 6B). S401 is close to the surface of PR65 in the PP2A holoenzyme and at the interface where PR65 interacts with the PP2A catalytic (C) subunit between HEAT repeat 10-15 (Supplemental Fig. 
6C). Interestingly, the putative PR65 NES is located in the inter-repeat loop between HEAT domains 10 and 11 and in close proximity to S401 within HEAT repeat 10 (106), and close to the outside boundary of the PP2A holoenzyme possibly more accessible for phosphorylation by ATM kinase (Supplemental Fig. 6C).

To confirm that PR65 and CRM1 interact in the cell, we carried out a proximity ligation assay (PLA). PR65-CRM1 proximity was observed as a fluorescent signal in untreated and irradiated WT cells with more punctate perinuclear appearance after irradiation. As expected, the PR65-CRM1 interaction was disrupted when cells were pretreated with LMB (Fig. 8D). Altogether, these results suggest that PR65 is exported to the cytoplasm by CRM1 after S401 is phosphorylated.

Because PP2A is such an abundant protein constituting $\sim 1 \%$ of total protein in a mammalian cell and involved with regulating many processes throughout the cell it is technically challenging to follow a PR65 sub-population engaged in one specific process out of many in the cell using immunostaining or standard fluorescence protein fusions because of preexisting background. Therefore, we transfected photoactivatable plasmid constructs pPAmCherry-PR65 WT, S401A and S401D into HEK293 cells and demonstrate similar expression levels as endogenous PR65 (Supplemental Fig. 7). Laser photo-activation to a specific sub-cellular location such as the nucleus would allow for following the shuttling of PR65 in live cells after DNA damage with less interference from unrelated PR65 events. Forty-eight hours post transfection a subnuclear Region-of-Interest (ROI) was photo-activated with a $405 \mathrm{~nm}$ laser to activate PAmCherry-PR65 WT. We determined that laser alone was sufficient to cause DNA damage without including Hoechst 33528 to the medium based on $\gamma-\mathrm{H} 2 \mathrm{AX}$ and 
phospho-KAP1 (S824) foci formation although the addition of dye increased DNA damage and seemed to enhance shuttling (Fig. 9A, Supplemental Fig. 8) with pannuclear PAmCherry-PR65 WT migrating to the nuclear membrane within minutes after photo-activation (Fig. 9B, C). We then confirmed our previous results with Flagimmunofluorescence (see Fig. 7), now using PAmCherry as reporter, that PR65-WT was exported from the nucleus to the cytoplasm whereas S401A was not, and PR65S401D remained in the cytoplasm (Fig. 9D, E, Supplemental Fig. 9). We plotted the nuclear to cytoplasmic ratios $(\mathrm{N} / \mathrm{C})$ of the collected fluorescence signals. With WT cells, the N/C was as high as 0.05 initially and declined over several minutes (Fig. 9E, Supplemental Table 2), suggesting that PAmCherry-PR65 was nuclear early after DNA damage and photo-activation and quickly shuttled to the cytoplasm. The N/C ratio in the S401A and the S401D cells were initially $30-60 \%$ less $(0.015-0.03)$ and remained the same throughout the experiment, suggesting that PR65-S401A and -S401D were statically nuclear and cytoplasmic, respectively (Fig. 9E, left panel). These results are consistent with the notion that nuclear PR65 is exported to the cytoplasm after DNA damage in a pS401-dependent manner. Additionally, in support of the physical presence of mutant PR65 in nucleus or cytoplasm, presumably with altered PP2A substrate specificity, is the distinct pattern of survival of MEFs after transfection with plasmids overexpressing different B regulatory subunits (Supplemental Fig. 10). We found a similar pattern of tolerability when $B 55 \alpha, B 56 \alpha$, or B56 $\beta$ were overexpressed in WT and S401 mutant cells, whereas a dramatically varied response was seen with $B 56 \gamma 1, B 56 \gamma 3, B 568$, and $B 56 \varepsilon$, suggesting that PR65 has preferential $B$ subunit partners depending on S401 status. 
To determine whether the putative PR65 NES sequence functions, we generated photoactivatable plasmid constructs, pPAmCherry-PR65 WT, S401A, and S401D, also having the L373A alteration. When HEK293 cells were transfected with pPAmCherryPR65 WT/L373A and then photo-activated, the initial N/C fluorescence intensity ratio was 0.10 , twice the ratio seen with WT, and the signal did not decline over time except at $>170 \mathrm{~s}$ that might represent photo bleaching (Fig. 9E, right panel, Supplemental Table 2). The S401A and S401D mutants were less affected by the L373A addition, but, interestingly, the flat N/C ratios seem to come more together compared with either S401 alteration alone. Altogether, our results show that ATM kinase serves as a gatekeeper for regulating the initial DDR by phosphorylating PR65 at S401 exposing a buried NES to CRM1 and resulting in nuclear export of PR65. 


\section{DISCUSSION}

PP2A plays a major role in numerous cellular processes and has been associated with regulating the DDR and DNA repair $(25,27,29,30)$. ATM kinase phosphorylates PR65 at S401, which is associated with dysfunctional neuronal chromatin deacetylation by HDAC4 resulting in neurodegeneration in ataxia telangiectasia (31). Previous work by our group has explored how DSB signaling through ATM converges on pro-survival signaling through AKT (21). Negative regulation of AKT by PP2A is well-established $(107,108)$. Herein we report that phosphorylation of a single, critical amino acid residue (S401) in the PR65 subunit is important for cell survival, DNA damage signaling, and DNA repair.

Our molecular modeling simulations suggest that phosphorylation of S401 causes the horseshoe-shaped scaffolding subunit PR65 to undergo a conformational change. This leads to the disassociation of the ATM-PR65 complex, as previously reported (25), which, herein is supported by our co-immunoprecipitation results. We also show that ATM, AKT and PP2A-C no longer interact with S401-phosphorylated PR65 or the analogous phospho-mimetic mutant. Impaired interaction of S401D with PP2A-C could therefore fundamentally alter PP2A phosphatase activity, substrate targeting, and/or cellular localization. A reciprocal immunoprecipitation experiment might resolve whether AKT is part of the PR65-ATM complex or is a separate PR65-AKT complex.

Interestingly, PR65 and ATM both have many HEAT repeats, 15 and 49, respectively. HEAT repeats consist of pairs of interacting, anti-parallel helices linked by flexible "intraunit" loops believed to form dynamic structures and elastic connectors that link force and catalysis $(106,109)$. Additionally, CRM1 has 19 HEAT repeats known to participate 
in the formation of a nuclear export complex (110). It is tempting to speculate that these HEAT repeats are involved in a dynamic "hand-over and exchange" process swapping partners during the DDR and resulting in nuclear export of PR65 expected to quickly alter the nuclear phospho-protein landscape and maintain a state of elevated phosphorylation in the nucleus immediately following DNA damage. Presumably, PR65 then shuttles back into the nucleus to turn off the DDR by dephosphorylating proteins targeted directly or indirectly by ATM phosphorylation. Clearly, this is a simplified scenario since several other S/T protein phosphatases such as PP1, PP4 and PP6 have also been linked to the DDR (111). However, it is well established that these phosphatases perform redundant functions by acting either as the primary phosphatase or secondary backup to avoid cellular crises to occur (e.g., during mitosis).

We, and others, have previously reported that an ATM-dependent phosphatase negatively controls AKT (S473) dephosphorylation in response to insulin and radiation $(21,112)$. We hypothesized that ATM mediated phosphorylation of PR65 at S401 might alter PP2A phosphatase activity thereby increasing AKT S473 phosphorylation after such stimulation which can be blocked by an ATM kinase inhibitor (21). In the present study, we found that AKT phosphorylation was elevated in S401D mutants relative to the WT and S401A counterparts. However, in response to radiation, the increase in AKT (S473) phosphorylation was impaired in S401D as well as S401A cells. We know that the DDR initially takes place primarily in the nucleus, whereas insulin signaling initiates at the plasma membrane through activation of insulin receptor and with AKT present in both compartments. It is likely that AKT is differentially regulated in the nucleus and cytoplasm by PP2A. This could be due to reduced PP2A activity and/or 
inappropriate cellular location of PP2A impairing the normal execution of the DDR. That the response to insulin and radiation are different might be expected because radiation likely produce more pleiotropic effects on signaling than insulin (69). This may be through association with different B subunits, and as demonstrated herein, in a coordinated PP2A nuclear-cytoplasmic shuttling process to spatio-temporally regulate the DDR as well as cell growth.

$\gamma-\mathrm{H} 2 \mathrm{AX}$ is present at DSBs in the nucleus. From our PR65 localization study, we observed that S401D was retained in the cytoplasm in cells exposed to radiation whereas the WT, and to a lesser extent S401A, was found in both the cytoplasm and nucleus. Thus, cytoplasmic sequestration of PR65-S401D possibly makes cells impaired in nuclear $\gamma-\mathrm{H} 2 \mathrm{AX}$ dephosphorylation. We have also shown that S401D cells are less efficient in DSB repair, accumulate chromosomal abnormalities and show reduced survival after DNA damage. On the other hand, S401A cells have much greater end joining activity compared to WT cells in vitro, which is in line with the idea that DNA repair cannot shut off. We speculate that this is due to the inhibition of nuclear export of PR65. Furthermore, since PP2A is essential for the radiation G2/M checkpoint (29), the decreased ability of PR65 to shuttle and retain proper PP2A activity in a strict spatiotemporal manner would likely result in checkpoint failure and mitotic catastrophe.

Most notably, both in vitro and in vivo studies suggest that DSB repair is fundamentally altered in the S401 mutants. Future studies will determine in more detail what type of DSB repair is engaged when both c-NHEJ and HRR are compromised as is the case in S401D cells. Most likely, a micro-homology type of DSB repair such as TMEJ or SSA is involved to rescue cell survival based on our preliminary analyses. 
As to the mechanism how PR65 S401 alterations might affect cell growth and DNA repair in a reciprocal manner, we propose a model in which PP2A together with ATM might serve as gatekeeper of the DDR (Fig. 10). For ATM-directed activation of the DDR to occur through a phosphorylation cascade, the phosphatase, in this case PP2A, must initially be inactivated to maintain a phosphorylated protein landscape that subsequently has to be reactivated by removing phosphates to reenter the cell cycle and resume growth. If the DNA damage is sub-lethal, DNA repair is mostly completed within a relatively short time window of 6-12 hours (113). Interestingly, ATM and PP2A form an auto-regulatory, yin yang/kinase-phosphatase relationship serving as a potential on-off switch of the DDR. Based on our findings and by others, PP2A is likely having the upper hand in this relationship since inhibitors of PP2A activate the ATM kinase, resulting in a pseudo-DDR response without significant DNA damage (25). There are many examples of this regulatory hierarchy in kinase-phosphatase signaling pathways (114). Nuclear export seems a fast, efficient means of temporarily removing PP2A from sites of DNA repair. Once repair is complete, PP2A can shuttle back to the nucleus to remove protein phosphates and reset the cell for baseline growth and activity. Therefore, PP2A (along with other phosphatases) must coordinate and balance DNA repair and cell survival with apoptosis resulting from insurmountable cellular damage. Our model incorporates the ATM-PP2A "on-off" switch for the DDR coupled with cell recovery and growth by ATM and PP2A shuttling between the nucleus and cytoplasm and, together, acting as a cellular rheostat for DNA damage and growth control. 
Our results suggest that the improper localization of PP2A/PR65 and, thus, untimely dephosphorylation of critical phospho-proteins result in a shift in the quality of DSB repair though without dramatic impact on survival. The most straightforward interpretation of our results is that DSB repair is re-wired in a manner that substitute cNHEJ and HRR with some other homology-based DNA repair to maintain viability after DNA damage. Regardless, because the only difference between the three cell populations used in our study is the PR65 S401 alterations, this cell system could be a rich source for dissecting DNA repair and growth responses regulated by PP2A that would otherwise not be possible with general DNA damaging treatments, which would trigger a plethora of difficult-to-dissect cellular responses. 


\section{Figure Legends}

Figure 1. Generation of PR65 KO MEFs complemented with S401 mutants. (A) Targeting strategy for generation PR65 conditional knockout (CKO) MEFs. Expression of Cre recombinase in these PR65-CKO MEFs would generate a PR65 KO MEFs by deletion of exon 5 and 6. PR65-CKO MEFs were infected with retroviral vector pMIGAalpha, expressing Flag-tagged PR65 WT, S401A or S401D mutant in addition to GFP. This was followed by Infection of cells with Ad-CMV-Cre recombinase to knock out the endogenous PR65 alleles. (B) Genomic DNA was isolated from PR65 KO MEFs (WT, S401A and S401D) and PCR amplified. PCR screening with primers P1, P2, and P3 shown in (A) generated a 263-bp product for WT allele and a 417-bp product for the CKO allele. In cells where Cre was expressed, primer pair P2 and P3 generated a 587bp product for the KO PR65 allele. (C) PR65 is essential for the survival of MEFs. PR65 CKO MEFs were infected with Ad-CMV-Cre recombinase or not. Cells were stained with crystal violet after one week to assess cell viability. PR65-CKO MEFs infected with AdCMV-Cre recombinase did not survive whereas PR65 flox/E64G MEFs did. (D) Plasmid map of pMIG-Aalpha WT with mutations marked (top) and expression of GFP in WT, S401A, S401D, and E64G MEFs with endogenous PR65 alleles KO (bottom). Scale bar is $10 \mu \mathrm{m}$. (E) Western blot verifying the Flag-PR65 expression of site-specific mutants and floxing of endogenous PR65 in MEFs. Left panel: Explanation of the protein bands seen by western blotting. Right panel: Whole cell extracts of GFP+ sorted PR65 WT, S401A and S401D cells infected with Ad-Cre and uninfected PR65-WT MEFs were separated on an SDS-PAGE gel and analyzed by western blotting using anti-PR65 antibody, anti-Flag and anti-GAPDH (loading control) antibodies. 
Figure 2. Phospho-mimetic S401D prevents the association of PR65 with ATM, AKT, and PP2A catalytic subunit. (A) Whole cell lysates of HEK293 cells stably expressing Flag-PR65 (WT, S401A and S401D) were immunoprecipitated with anti-Flag beads. Immunoprecipitates (left panel) and whole cell extracts (right panel) were separated on an SDS-PAGE gel and analyzed by western blotting using anti-Flag, antiATM, anti-AKT and anti-PP2A-C antibodies. (B) MD simulations suggests that a conformational change occur when S401 is phosphorylated. (Left) Top view of the in silico S401 phosphorylated PR65, after 50 ns, aligned to the original experimental structure (PDB ID 2IAE) with a root-mean square deviation (RMSD) of $8.05 \AA$. PR65 (pS401) is shown in magenta, with p-S401 represented as stick figures. PR65 from protein database (PDB ID 21AE) is shown in green. (Right) Top view of the unphosphorylated control structure of PR65 subunit, after 50 ns, aligned to the original experimental structure taken from PDB ID 2IAE (RMSD = 6.51 $\AA$ ). Unphosphorylated control PR65 is shown in cyan, and the original experimental structure shown in green. Molecular modeling studies also indicate that phosphorylation at S401 could cause a conformational change which could affect its association with a binding partner. (C) Two 50 ns molecular dynamics simulations were carried out using the S401 phosphorylated PR65 chain as well as the unphosphorylated structure. Comparing only the residues surrounding S401 (residues 375-454), the RMSD of the phosphorylated subunit and the unphosphorylated experimental structure was found to be $4.05 \AA$ while the RMSD of the control and the unphosphorylated experimental structure was found to be $2.55 \AA$. At the end of the simulation, the distance between $\alpha$-carbons for residues 
S401 and A431 for the unphosphorylated original structure, the S401 phosphorylated structure, and control structure were $10.873 \AA, 15.321 \AA$ and $9.498 \AA$, respectively (top pane/). Initially, there was a readjustment phase in the phosphorylated MD simulation in the region surrounding the phosphorylated S401 residue after which it stabilized at 18 ns (bottom panel). Altogether this result suggests that phosphorylation of PR65-S401 could affect the interaction with its binding partners and result in the dissociation of the holoenzyme

Figure 3. Growth and irradiation signaling is impaired in PR65 S401 mutant cells. (A) AKT phosphorylation is increased in S401D cells after insulin stimulation. MEFs (WT, S401A and S401D) were serum starved for 16 hours and then treated with insulin for 15-, 30- or 60-min. Whole cell extracts were separated on an SDS-PAGE gel and analyzed by western blotting with anti-pAKT (S473), anti-AKT, anti-GAPDH (loading control) antibodies (left panel). Western blots were quantified using Image Studio Lite. p-AKT (S473) levels were normalized to total AKT protein levels (right panel). (B) S401D cells have increased growth rate relative to S401A and WT. MEFs were grown at different dilutions in 96-well plates. Cells were analyzed by Cell titer-Glo® at 2, 5, and 7 days to determine growth. Data points; Growth, Relative Luminescence Units (RLU). Error bars; mean \pm SEM $(n=3)$. Statistical analysis was carried out using unpaired, twotailed $t$-tests. $P$-values expressed as ${ }^{*}(P<0.05)$ and ${ }^{* *}(\mathrm{p}<0.01)$ were considered significant. At 7 days, WT vs. S401D; $p=0.0026$, S401A vs. S401D; $p=0.0118$. (C) ERK phosphorylation is elevated in S401D cells. Whole cell extracts of MEFs were separated on an SDS-PAGE gel and analyzed by western blotting using anti-pAKT, anti-AKT, anti- 
pERK and anti-ERK antibodies. PAKT (S473) levels were normalized to total AKT protein levels and anti-pERK (T202/Y204) levels were normalized to total ERK levels. Increased pERK levels correlate with increased growth of S401D cells. (D) S401 cells show dampened response to irradiation. MEFs were exposed to $2 \mathrm{~Gy}$ of ionizing radiation and collected after $5,15,30$ and 60 minutes. Whole cell extracts were separated on an SDS-PAGE gel and analyzed by western blotting using anti-pAKT, anti-AKT, anti- $\gamma-\mathrm{H} 2 \mathrm{AX}$, and anti-GAPDH antibodies (top panel). Western blots were quantified using Image Studio Lite (Li-Cor). p-AKT levels were normalized to total AKT protein levels and $\gamma-\mathrm{H} 2 \mathrm{AX}$ levels were normalized to GAPDH (bottom panels).

Figure 4. PR65 mutants accumulate mitotic aberrations leading to mitotic catastrophe and radiosensitization. (A, B and C) S401 mutant cells have defective G2/M checkpoint. MEFs were exposed to $5 \mathrm{~Gy}$ of ionizing radiation, fixed after 24 hours and stained with DAPI to label nuclei. Mitotic cells and total number of cells were counted. The mitotic index was calculated by dividing the number of cells undergoing mitosis in a population to the number of cells not undergoing mitosis. Five separate fields were assessed for each group Scale bar is $10 \mu \mathrm{m}$. (B). Mitotic indices were quantified from A. Error bars; mean \pm SEM Statistical analysis was carried out using unpaired, two-tailed $t$ - tests. $P$ values expressed as ${ }^{*}(P<0.05)$ were considered significant; $n$.s. not significant $(P>0.05)$. WT: Untreated vs IR; $p=0.0251$. (C) Aberrant mitoses were quantified from A. Five separate fields were assessed for each group. Error bars; mean \pm SEM. Statistical analysis was carried out using unpaired, two-tailed $t$ - tests. $P$ values expressed as ${ }^{*}(P<0.05)$ were considered significant; $n$.s. not 
significant $(P>0.05)$. IR: WT vs S401D; $p=0.0228$ (D) MEFs were infected with a H2BmCherry virus construct to monitor chromatin structure in mitosis. MEFs were exposed to $5 \mathrm{~Gy}$ and subjected to live-cell video imaging for 8 hours on the Zeiss Cell Observer Spinning Disc confocal microscope. Representative still images (left panel) and quantified mitotic length (right panel) Scale bar is $10 \mu \mathrm{m}$. E) S401D cells quickly exit mitosis after mitotic synchronization. Cells were arrested in M phase with 16 hours of nocodazole treatment. Mitotic cells were collected by shake-off, reseeded in complete media, and collected after 1, 2 and 4 hours for western blotting with anti-pPLK1 (T210) antibody, a marker of mitotic exit (F), and plotted (G). Faster pPLK1 dephosphorylation occurs in S401D cells expected to speed up mitotic exit. Altered PP2A-S401D target specificity/activity might explain quicker exit from mitosis. (H) S401D cells show delayed replication restart. MEFs were treated with $1 \mathrm{mM}$ of hydroxyurea $(\mathrm{HU})$ for 2 hours followed by drug wash-out and collected at 0,1 , and 6 hours after wash-out. Western blotting with antibodies specific for pRPA (S4/S8) and $\gamma-\mathrm{H} 2 \mathrm{AX}$ normalized to total GAPDH. (I) S401 mutant cells are more radiosensitive than wild-type MEFs. MEFs were exposed to 1, 2 or 5 Gy and radiosurvival determined at 10 days. Surviving fractions were determined by crystal violet staining and colony counting. Data points, surviving cells plotted as fraction of control (- irradiation). Results are presented as mean \pm SEM, $(n=4)$. Statistical analysis was carried out with longitudinal ANOVA on the survival fractions. $P$-values expressed as ${ }^{*}(P<0.05)$ were considered significant. WT vs. $D, p=$ 0.0302 . 
Figure 5. DSB repair is abnormally high in S401A cells while being suppressed in

S401D. (A) Scheme for in vivo end joining (EJ) assay. Linearized Tomato expression plasmid was transfected into MEFs followed by quantification of RFP+ cell (Nexcelom Cellometer) and PCR. (B) Quantification of EJ by RFP flow cytometry (top panel). S401A cells have super-active EJ at later time point after transfection whereas S401D cells have suppressed EJ throughout. qPCR assay was carried out to determine early EJ (bottom panel). EJ in S401D cells was suppressed by $75 \%$ at 4 hours in line with the results by RFP flow. (C) In vitro end joining. End joining of an internally labeled $\left(^{*}\right)$ plasmid substrate with partially complementary ends in MEF extracts (left panel). Following incubation for the indicated times, substrates were cut with BstXI and Taql and analyzed on sequencing gels (middle panel). Gap-filling products (42-base fragment) and resection-based products (34-base fragment) were quantified. The 24and 16-base fragments are the corresponding head-to-head end joining products. Graph shows sum of the major 34-base fragments in each case (right panel). (D) Mutant S401 cells show aberrant IR-induced foci with delayed disappearance of 53BP1 and $\gamma$-H2AX foci in S401 mutant cells (left panel). MEFs were seeded in a chambered slide. Cells were exposed to 2 Gy of ionizing irradiation and fixed after 0.5 and 5 hours. Cells were immuno-stained with anti-53BP1 and anti- $\gamma-\mathrm{H} 2 \mathrm{AX}$ and counterstained with DAPI (blue) to label nuclei. Representative images 53BP1 (Green), $\gamma$-H2AX (red) and DAPI (blue). Images were acquired on Zeiss LSM 710 confocal microscope at 63x power. Repair foci remain significantly longer in S401A and -D (esp. D) than in WT cells suggesting aberrant DSB repair. Delayed disappearance of 53BP1 (top, right panel) and $\gamma-\mathrm{H} 2 \mathrm{AX}$ (bottom, right panel) foci in S401 cells post irradiation. Error bars; mean \pm SEM. 
Statistical analysis was carried out using unpaired, two-tailed $t$ - tests. p-values are shown as ${ }^{* *}(p<0.005)$ is considered significant. Scale bar is $10 \mu \mathrm{m}$. (E) S401A and D cells are impaired in homologous recombination using the DR-GFP assay (top, left panel). PR65-DR-GFP (WT, S401A and S401D) cells were infected with Ad-Scel (bottom, right panel) or not (top, right panel) and 72 hours later, cell populations analyzed by GFP flow cytometry after incubation in $0.1 \%$ Triton-X-100 in PBS. Results (bottom, left panel) are presented as mean \pm SEM $\left(30,000\right.$ events), $n=3,{ }^{*} p<0.05$ relative to WT Scale bar is $10 \mu \mathrm{m}$. (F) Reduced Rad51 and pRPA foci in S401 mutants post-CPT treatment. MEFs were treated with $100 \mathrm{nM}$ of CPT for 6 hours, fixed and immunostained with anti-pRPA32(S4/S8), anti-Rad51 antibodies, and counterstained with DAPI (blue). Representative images Rad51 (Red), pRPA (Green) and DAPI (blue) images acquired on a Zeiss LSM 710 confocal microscope at 63x power (left panel). Quantification of Rad51 and pRPA32 (S4/8) foci (right pane/s). Rad51 foci per cell were counted in 5 separate fields. Error bars; mean \pm SEM. Statistical analysis was carried out using unpaired, two-tailed $t$-tests. $P$-values expressed as ${ }^{* * *}(\mathrm{p}<0.005)$ and ${ }^{\#}(\mathrm{p}<$ 0.001) were considered significant. For Rad51 foci, WT vs. S401A; $p=0.0008$, WT vs. S401D; $p=0.0011$. For $p R P A$ foci, WT vs. S401D; $p=0.0443$.

Figure 6. CRISPR-Cas9 mediated DSB repair. (A) DSB repair at the Rosa26 (Chr 6) locus. Xbal digestion removes background PCR DNA without indels (no NHEJ)* and leaves behind the $\sim 221$-bp PCR fragments with indels ${ }^{* *}$ (top panel). Primers: Chr6 Rosa26-F/Rosa26-R (47). The Rosa26 CRISPR-Cas9 gRNA target is spanning an Xbal-I restriction site (bottom panel). Thus, an indel would eliminate the Xba-I site. (B) 
Rosa26 (Chr 6) and H3f3b (Chr 11) translocation between DSBs generated by dual CRISPR-Cas9 cleavage. Primers: Chr6 Rosa26-F/Chr11 H3f3b-R. Major indels at translocation junctions are seen only in D cells* although translocation is seen in all three cell mixes (left panel). PCR fragments were cloned into pGEM-T Easy and sequenced (right panel). (C) Fragment size distributions of Rosa26-H3f3b translocation amplification products as determined by (a) $2 \%$ agarose gel electrophoresis and (b)-(i) high-speed atomic force microscopy (AFM). Four samples were amplified: wild-type (WT, $n=11,303$ measured strands), S401A ( $n=6,530)$, S401D $(n=12,160)$, and a synthetic fragment (Syn, $n=6,035)$ of equivalent length and sequence as WT. In (b)(e), the normal probability density of amplicon lengths for each sample is shown as well as a Gaussian mixture model fit to each distribution. The probability density function of each single Gaussian fit is shown as a smooth colored curve, with that curve's population proportion given in the same color. Also, above the probability density plots, the mean of each Gaussian fit is plotted as a hollow circle, and the $95 \%$ confidence interval of the mean is shown as a horizontal line $(\mathrm{Cl}$ calculated from a bootstrap simulation with $n=10,000$ ) (Supplemental Table 1). In (f)-(i), high-speed AFM images of DNA amplicons from each sample are shown with $200 \mathrm{~nm}$ scale bars and lighter colors representing greater height. In frame (b), arrows point from the two main distributions to imaged strands in frame (f) that belong to the indicated population. In frame (i), a higher magnification of a single strand is shown. The red backbones shown on some amplicons represent the measured lengths of those amplicons; if an amplicon has no red backbone, it did not meet the image analysis algorithm's quality standard and was not measured. 
Figure 7. Radiation-induced nuclear export of WT PR65 is mimicked by S401D. (A) Wild-type MEFs grown in chamber slides were irradiated with $10 \mathrm{~Gy}$ with or without leptomycin B (LMB) present $(1 \mathrm{ng} / \mathrm{ml} ;-3$ hour) in the medium. Cells were fixed at 30 and 60 minutes after irradiation and stained with anti-Flag antibody to detect PR65 (red) and counterstained with DAPI (blue). Images were acquired on Zeiss LSM 710 confocal microscope at 40x power. Irradiation results in cytoplasmic accumulation of Flag-PR65 seen best at 60 min (top panel). LMB blocks PR65 nuclear-cytoplasmic shuttling as shown best by the decreased nuclear and increased cytoplasmic accumulation at 30 min (bottom panel) Scale bar is $10 \mu \mathrm{m}$. (B) MEFs were grown on chamber slides and irradiated with $10 \mathrm{~Gy}$, fixed at $10 \mathrm{~min}$ and processed as in (A). S401D markedly showed no nuclear staining prior to or post irradiation whereas both WT and S401A cells have Flag-PR65 nuclear and cytoplasmic staining Scale bar is $10 \mu \mathrm{m}$. (C) Model for radiationinduced nuclear export of PR65 mediated by S401 phosphorylation (see text).

Figure 8. PR65 and CRM1 physically interact. (A) CRM1 forms a complex with PR65 as shown by co-immunoprecipitation with Flag-CRM1. HEK293 cells were transfected with Flag-CRM1, mRuby2-PR65 and RanQ69L expression plasmids followed by wholecell immunoprecipitation at 72 hours using Flag-beads and western blotting with antiPR65 and -Flag antibodies. (B) HINT analysis of CRM1-PR65 and CRM1-Snurportin 1 interactions suggest that CRM1-PR65 hydrophobic interaction is stronger than between CRM1 and Snurportin 1. (C) Modeling suggests that CRM1 (red) interacts with the $\mathrm{L}_{365} \mathrm{LPLFLAQL}{ }_{373}$ - motif of PR65 (cyan) with S401 in the vicinity of the binding interface (yellow spheres). (D) PR65 and CRM1 co-localize at the cytoplasmic-nuclear 
membrane interface. Proximity Ligation Assay (PLA) shows that PR65 and CRM1 interact within 30-40 nm prior to irradiation with 10 Gy. After irradiation, this interaction was more punctated at the cytoplasmic-nuclear membrane. Pre-treatment with LMB (1 $\mathrm{ng} / \mathrm{ml}$ ) eliminates any sign of PR65-CRM1 interaction Scale bar is $10 \mu \mathrm{m}$.

Figure 9. DNA damage triggers PR65 movement to the nuclear membrane. (A) Laser photo-activation of nuclear PR65 in HEK293 cells - adding Hoechst 33258 results in increased DNA damage and DSBs as indicated by the increased $\gamma-\mathrm{H} 2 \mathrm{AX}$ nuclear staining Scale bar is $10 \mu \mathrm{m}$. (B) Nuclear PR65 migrates to the nuclear membrane after DNA damage. Time-lapse video shows that PR65 within minutes after $405 \mathrm{~nm}$ laser sub-nuclear irradiation (white square) is; 1) activated (mCherry signal increases) and 2) migrates to the nuclear membrane. The inclusion of Hoechst 33258 seems to increase the speed by which this movement occurs (green arrow) Scale bar is $10 \mu \mathrm{m}$. (C) Simplified model for PR65 accumulation at the nuclear membrane and export via the CRM1 nuclear protein complex. (D) pPAmCherry-PR65-WT is exported to the cytoplasm after nuclear irradiation. HEK293 cells were transfected with pPAmCherryPR65 (WT, S401A and S401D) in glass bottom dishes and $48 \mathrm{~h}$ post-transfection subnuclear ROI ( $4 \mu \mathrm{m} \times 4 \mu \mathrm{m})$, marked by the squares, were photoactivated by $405 \mathrm{~nm}$ laser $(242.8 \mu \mathrm{J})$ and followed over time $(<300 \mathrm{~s})$ by live cell imaging using a Zeiss LSM 710 microscope with images acquired at $63 x$. WT-PR65 is exported from nucleus to cytoplasm over time whereas PR65-S401A is retained in the nucleus and PR65-S401D remains cytoplasmic Scale bar is $10 \mu \mathrm{m}$. (E) PR65 NES mutation dramatically alters the behavior of PR65. In WT cells, the N/C was as high as 0.05 initially and declined over 
several minutes, suggesting that PAmCherry-PR65 was nuclear early after DNA damage and photo-activation and quickly shuttled to the cytoplasm. The N/C ratio in the S401A and the S401D cells were initially 30-60\% less $(0.015-0.03)$ and remained the same throughout the experiment, suggesting that PR65-S401A and -S401D were statically nuclear and cytoplasmic, respectively (left panel). When HEK293 cells were transfected with pPAmCherry-PR65 WT/L373A and photo-activated, the initial N/C fluorescence intensity ratio was 0.10 , twice the ratio seen with WT, and the signal did not decline over time except at $>170 \mathrm{~s}$ that might represent photo bleaching. The S401A and S401D mutants were less affected by the L373A addition, but, interestingly, the flat N/C ratios seem to come more together compared with S401 alteration alone (right panel). One-way ANOVA was performed on the time series. $p<0.0001$ for WT vs S401A, WT vs S401D, NES vs NES-S401A and NES vs NEs-S401D.

Figure 10. Model for ATM-PP2A mediated regulation of the DDR and cell growth spatio-temporal (de)phosphorylation and nuclear-cytoplasmic shuttling by ATM and PP2A controls the DDR “ON-OFF” switch. ATM phosphorylates many proteins in response to IR including $\mathrm{H} 2 \mathrm{AX}$ at S139 and PR65 at S401 thereby initiating the DDR and triggering cell cycle checkpoints and growth arrest, and transiently inhibiting DSB repair. Within minutes, phosphorylated PR65 translocates to the cytoplasm that reduces PR65 activity in the nucleus allowing the DDR with its highly phosphorylated protein landscape to run its course. At the same time, AKT and other growth-promoting factors and signaling pathways are inhibited by the increased PR65 activity in the cytoplasm while the damage is assessed and repaired. Soon after the DDR is beginning to 
subside, PR65 might shuttle back to the nucleus where it increases PR65 activity and begin to engage DSB repair while $\gamma-\mathrm{H} 2 \mathrm{AX}$ and other DDR targets are dephosphorylated. When repair is completed and cells resume cycling, growth recovers. 


\section{Acknowledgements}

Services in support of the research project were provided by the VCU Massey Cancer

Center Transgenic/Knockout Mouse Core, the Microscopy Shared Resource, the Flow Cytometry Shared Resource, and the Cancer Mouse Model Core supported, in part, with funding from NIH-NCI Cancer Center Support Grant P30 CA016059.

\section{Reporting summary}

Further information on research design is available in the Nature Research Reporting Summary linked to this article.

\section{Data availability}

All the data supporting the findings of this study are either available within the paper and its Supplementary Information files or can be obtained from the authors upon request. 


\section{References}

1. Janssens V, Goris J. Protein phosphatase 2A: a highly regulated family of serine/threonine phosphatases implicated in cell growth and signalling. Biochem J 2001;353(Pt 3):417-39.

2. Millward TA, Zolnierowicz S, Hemmings BA. Regulation of protein kinase cascades by protein phosphatase 2A. Trends Biochem Sci 1999;24(5):186-91.

3. Wang F, Zhu S, Fisher LA, Wang W, Oakley GG, Li C, et al. Protein interactomes of protein phosphatase 2A B55 regulatory subunits reveal B55-mediated regulation of replication protein $A$ under replication stress. Sci Rep 2018;8(1):2683.

4. Eichhorn PJ, Creyghton MP, Bernards R. Protein phosphatase 2A regulatory subunits and cancer. Biochim Biophys Acta 2009;1795(1):1-15.

5. Mayer-Jaekel RE, Baumgartner S, Bilbe G, Ohkura H, Glover DM, Hemmings BA. Molecular cloning and developmental expression of the catalytic and 65-kDa regulatory subunits of protein phosphatase 2A in Drosophila. Molecular biology of the cell 1992;3(3):287-98.

6. Mayer-Jaekel RE, Hemmings BA. Protein phosphatase 2A--a 'menage a trois'. Trends Cell Biol 1994;4(8):287-91.

7. Walter G, Ferre F, Espiritu O, Carbone-Wiley A. Molecular cloning and sequence of cDNA encoding polyoma medium tumor antigen-associated 61-kDa protein. Proceedings of the National Academy of Sciences of the United States of America 1989;86(22):8669-72.

8. Hemmings BA, Adams-Pearson C, Maurer F, Muller P, Goris J, Merlevede W, et al. alpha- and beta-forms of the 65-kDa subunit of protein phosphatase 2A have a similar 39 amino acid repeating structure. Biochemistry 1990;29(13):3166-73.

9. Ruediger R, Roeckel D, Fait J, Bergqvist A, Magnusson G, Walter G. Identification of binding sites on the regulatory A subunit of protein phosphatase 2A for the catalytic $C$ subunit and for tumor antigens of simian virus 40 and polyomavirus. Mol Cell Biol 1992;12(11):4872-82.

10. Zhou J, Pham HT, Ruediger R, Walter G. Characterization of the Aalpha and Abeta subunit isoforms of protein phosphatase 2A: differences in expression, subunit interaction, and evolution. Biochem J 2003;369(Pt 2):387-98.

11. Zolnierowicz S, Csortos C, Bondor J, Verin A, Mumby MC, DePaoli-Roach AA. Diversity in the regulatory B-subunits of protein phosphatase $2 A$ : identification of a novel isoform highly expressed in brain. Biochemistry 1994;33(39):11858-67.

12. Seshacharyulu P, Pandey P, Datta K, Batra SK. Phosphatase: PP2A structural importance, regulation and its aberrant expression in cancer. Cancer Lett 2013;335(1):9-18.

13. Shiloh Y. ATM and related protein kinases: safeguarding genome integrity. Nat Rev Cancer 2003;3(3):155-68.

14. Valerie K, Povirk L. Regulation and mechanisms of mammalian double-strand break repair. Oncogene 2003;22:5792-812.

15. Lavin MF. Ataxia-telangiectasia: from a rare disorder to a paradigm for cell signalling and cancer. Nat Rev Mol Cell Biol 2008;9(10):759-69. 
16. Shiloh Y, Kastan MB. ATM: genome stability, neuronal development, and cancer cross paths. Adv Cancer Res 2001;83:209-54.

17. Matsuoka S, Ballif BA, Smogorzewska A, McDonald ER, 3rd, Hurov KE, Luo J, et al. ATM and ATR substrate analysis reveals extensive protein networks responsive to DNA damage. Science 2007;316(5828):1160-6.

18. Abraham RT. Cell cycle checkpoint signaling through the ATM and ATR kinases. Genes Dev 2001;15(17):2177-96.

19. Shiloh Y, Ziv Y. The ATM protein kinase: regulating the cellular response to genotoxic stress, and more. Nature reviews Molecular cell biology 2013;14(4):197-210.

20. Peng A, Maller JL. Serine/threonine phosphatases in the DNA damage response and cancer. Oncogene 2010;29(45):5977-88.

21. Golding SE, Rosenberg E, Valerie N, Hussaini I, Frigerio M, Cockcroft XF, et al. Improved ATM kinase inhibitor KU-60019 radiosensitizes glioma cells, compromises insulin, AKT and ERK prosurvival signaling, and inhibits migration and invasion. Mol Cancer Ther 2009;8(10):2894-902.

22. Cohen P, Klumpp S, Schelling DL. An improved procedure for identifying and quantitating protein phosphatases in mammalian tissues. FEBS Lett 1989;250(2):596-600.

23. Favre B, Turowski $P$, Hemmings BA. Differential inhibition and posttranslational modification of protein phosphatase 1 and 2A in MCF7 cells treated with calyculin-A, okadaic acid, and tautomycin. The Journal of biological chemistry 1997;272(21):13856-63.

24. Swingle M, Ni L, Honkanen RE. Small-molecule inhibitors of ser/thr protein phosphatases: specificity, use and common forms of abuse. Methods Mol Biol 2007;365:23-38.

25. Goodarzi AA, Jonnalagadda JC, Douglas P, Young D, Ye R, Moorhead GB, et al. Autophosphorylation of ataxia-telangiectasia mutated is regulated by protein phosphatase 2A. Embo J 2004;23(22):4451-61.

26. Li G, Elder RT, Qin K, Park HU, Liang D, Zhao RY. Phosphatase type 2Adependent and -independent pathways for ATR phosphorylation of Chk1. The Journal of biological chemistry 2007;282(10):7287-98.

27. Li HH, Cai X, Shouse GP, Piluso LG, Liu X. A specific PP2A regulatory subunit, B56gamma, mediates DNA damage-induced dephosphorylation of p53 at Thr55. Embo J 2007;26(2):402-11.

28. Jiang $\mathrm{Y}$. Regulation of the cell cycle by protein phosphatase $2 \mathrm{~A}$ in Saccharomyces cerevisiae. Microbiol Mol Biol Rev 2006;70(2):440-9.

29. Yan Y, Cao PT, Greer PM, Nagengast ES, Kolb RH, Mumby MC, et al. Protein phosphatase $2 \mathrm{~A}$ has an essential role in the activation of gamma-irradiationinduced G2/M checkpoint response. Oncogene 2010;29(30):4317-29.

30. Chowdhury D, Keogh MC, Ishii H, Peterson CL, Buratowski S, Lieberman J. gamma-H2AX dephosphorylation by protein phosphatase 2A facilitates DNA double-strand break repair. Mol Cell 2005;20(5):801-9.

31. Li J, Chen J, Ricupero CL, Hart RP, Schwartz MS, Kusnecov A, et al. Nuclear accumulation of HDAC4 in ATM deficiency promotes neurodegeneration in ataxia telangiectasia. Nat Med 2012;18(5):783-90. 
32. Chen W, Arroyo JD, Timmons JC, Possemato R, Hahn WC. Cancer-associated PP2A Aalpha subunits induce functional haploinsufficiency and tumorigenicity. Cancer research 2005;65(18):8183-92.

33. Pierce AJ, Johnson RD, Thompson LH, Jasin M. XRCC3 promotes homologydirected repair of DNA damage in mammalian cells. Genes Dev 1999;13(20):2633-8.

34. Beckta JM, Adams BR, Valerie K. DNA Damage Response in Human Stem Cells and Neural Descendants. Methods in molecular biology 2017;1599:375-90.

35. Beckta JM, Dever SM, Gnawali N, Khalil A, Sule A, Golding SE, et al. Mutation of the BRCA1 SQ-cluster results in aberrant mitosis, reduced homologous recombination, and a compensatory increase in non-homologous end joining. Oncotarget 2015;6(29):27674-87.

36. Ruediger R, Ruiz J, Walter G. Human cancer-associated mutations in the Aalpha subunit of protein phosphatase $2 \mathrm{~A}$ increase lung cancer incidence in Aalpha knock-in and knockout mice. Molecular and cellular biology 2011;31(18):3832-44.

37. Lei Y. Generation and culture of mouse embryonic fibroblasts. Methods Mol Biol 2013;1031:59-64.

38. Hickson I, Zhao Y, Richardson CJ, Green SJ, Martin NM, Orr Al, et al. Identification and characterization of a novel and specific inhibitor of the ataxiatelangiectasia mutated kinase ATM. Cancer Res 2004;64(24):9152-9.

39. Rosenberg E, Taher MM, Kuemmerle NB, Farnsworth J, Valerie K. A truncated human xeroderma pigmentosum complementation group A protein expressed from an adenovirus sensitizes human tumor cells to ultraviolet light and cisplatin. Cancer Res 2001;61(2):764-70.

40. Canman CE, Lim DS, Cimprich KA, Taya Y, Tamai K, Sakaguchi K, et al. Activation of the ATM kinase by ionizing radiation and phosphorylation of p53. Science 1998;281(5383):1677-9.

41. Vriend LE, Jasin M, Krawczyk PM. Assaying break and nick-induced homologous recombination in mammalian cells using the DR-GFP reporter and Cas 9 nucleases. Methods Enzymol 2014;546:175-91.

42. Povirk LF, Zhou RZ, Ramsden DA, Lees-Miller SP, Valerie K. Phosphorylation in the serine/threonine 2609-2647 cluster promotes but is not essential for DNAdependent protein kinase-mediated nonhomologous end joining in human wholecell extracts. Nucleic Acids Res 2007;35(12):3869-78.

43. Zhou T, Akopiants K, Mohapatra S, Lin PS, Valerie K, Ramsden DA, et al. Tyrosyl-DNA phosphodiesterase and the repair of 3'-phosphoglycolateterminated DNA double-strand breaks. DNA Repair (Amst) 2009;8(8):901-11.

44. Golding SE, Rosenberg E, Khalil A, McEwen A, Holmes M, Neill S, et al. Double strand break repair by homologous recombination is regulated by cell cycleindependent signaling via ATM in human glioma cells. J Biol Chem 2004;279(15):15402-10.

45. Golding SE, Rosenberg E, Neill S, Dent P, Povirk LF, Valerie K. Extracellular signal-related kinase positively regulates ataxia telangiectasia mutated, homologous recombination repair, and the DNA damage response. Cancer Res 2007;67(3):1046-53. 
46. Mateos-Gomez PA, Gong F, Nair N, Miller KM, Lazzerini-Denchi E, Sfeir A. Mammalian polymerase theta promotes alternative NHEJ and suppresses recombination. Nature 2015;518(7538):254-7.

47. Wyatt DW, Feng W, Conlin MP, Yousefzadeh MJ, Roberts SA, Mieczkowski P, et al. Essential Roles for Polymerase theta-Mediated End Joining in the Repair of Chromosome Breaks. Mol Cell 2016;63(4):662-73.

48. Sanjana NE, Shalem O, Zhang F. Improved vectors and genome-wide libraries for CRISPR screening. Nat Methods 2014;11(8):783-4.

49. Stringer BW, Day BW, D'Souza RCJ, Jamieson PR, Ensbey KS, Bruce ZC, et al. A reference collection of patient-derived cell line and xenograft models of proneural, classical and mesenchymal glioblastoma. Sci Rep 2019;9(1):4902.

50. Koebley SR, Reed J. Can a new microscopy platform help to improve clinical outcomes? Biotechniques 2018;65(5):250-51.

51. Mikheikin A, Olsen A, Leslie K, Russell-Pavier F, Yacoot A, Picco L, et al. DNA nanomapping using CRISPR-Cas9 as a programmable nanoparticle. Nat Commun 2017;8(1):1665.

52. Mikheikin A, Olsen A, Picco L, Payton O, Mishra B, Gimzewski JK, et al. HighSpeed Atomic Force Microscopy Revealing Contamination in DNA Purification Systems. Anal Chem 2016;88(5):2527-32.

53. Binnig G, Quate CF, Gerber C. Atomic force microscope. Phys Rev Lett 1986;56(9):930-33.

54. Picco LM, Dunton PG, Ulcinas A, Engledew DJ, Hoshi O, Ushiki T, et al. Highspeed AFM of human chromosomes in liquid. Nanotechnology 2008;19(38):384018.

55. Phillips JC, Braun R, Wang W, Gumbart J, Tajkhorshid E, Villa E, et al. Scalable molecular dynamics with NAMD. J Comput Chem 2005;26(16):1781-802.

56. MacKerell AD, Bashford D, Bellott M, Dunbrack RL, Evanseck JD, Field MJ, et al. All-atom empirical potential for molecular modeling and dynamics studies of proteins. J Phys Chem B 1998;102(18):3586-616.

57. Humphrey W, Dalke A, Schulten K. VMD: visual molecular dynamics. J Mol Graph 1996;14(1):33-8, 27-8.

58. de Vries SJ, van Dijk M, Bonvin AM. The HADDOCK web server for data-driven biomolecular docking. Nat Protoc 2010;5(5):883-97.

59. Dong X, Biswas A, Chook YM. Structural basis for assembly and disassembly of the CRM1 nuclear export complex. Nat Struct Mol Biol 2009;16(5):558-60.

60. Dong X, Biswas A, Suel KE, Jackson LK, Martinez R, Gu H, et al. Structural basis for leucine-rich nuclear export signal recognition by CRM1. Nature 2009;458(7242):1136-41.

61. Mashiach E, Schneidman-Duhovny D, Andrusier N, Nussinov R, Wolfson HJ. FireDock: a web server for fast interaction refinement in molecular docking. Nucleic Acids Res 2008;36(Web Server issue):W229-32.

62. Eugene Kellogg G, Abraham DJ. Hydrophobicity: is LogP(o/w) more than the sum of its parts? Eur J Med Chem 2000;35(7-8):651-61.

63. Sarkar A, Kellogg GE. Hydrophobicity--shake flasks, protein folding and drug discovery. Curr Top Med Chem 2010;10(1):67-83. 
64. Pallas DC, Shahrik LK, Martin BL, Jaspers S, Miller TB, Brautigan DL, et al. Polyoma small and middle $\mathrm{T}$ antigens and SV40 small $t$ antigen form stable complexes with protein phosphatase 2A. Cell 1990;60(1):167-76.

65. Rodriguez-Viciana P, Collins C, Fried M. Polyoma and SV40 proteins differentially regulate PP2A to activate distinct cellular signaling pathways involved in growth control. Proc Natl Acad Sci U S A 2006;103(51):19290-5.

66. Campbell KS, Auger KR, Hemmings BA, Roberts TM, Pallas DC. Identification of regions in polyomavirus middle $T$ and small $t$ antigens important for association with protein phosphatase 2A. J Virol 1995;69(6):3721-8.

67. Viniegra JG, Martinez N, Modirassari P, Hernandez Losa J, Parada Cobo C, Sanchez-Arevalo Lobo VJ, et al. Full activation of PKB/Akt in response to insulin or ionizing radiation is mediated through ATM. The Journal of biological chemistry 2005;280(6):4029-36.

68. Rodgers JT, Vogel RO, Puigserver P. Clk2 and B56beta mediate insulinregulated assembly of the PP2A phosphatase holoenzyme complex on Akt. Mol Cell 2011;41(4):471-9.

69. Valerie K, Yacoub A, Hagan MP, Curiel DT, Fisher PB, Grant S, et al. Radiationinduced cell signaling: inside-out and outside-in. Mol Cancer Ther 2007;6(3):789801.

70. Burma S, Chen BP, Murphy M, Kurimasa A, Chen DJ. ATM Phosphorylates Histone H2AX in Response to DNA Double-strand Breaks. J Biol Chem 2001;276(45):42462-7.

71. Ruvolo PP. The broken "Off" switch in cancer signaling: PP2A as a regulator of tumorigenesis, drug resistance, and immune surveillance. BBA Clin 2016;6:8799.

72. Wlodarchak N, Xing Y. PP2A as a master regulator of the cell cycle. Crit Rev Biochem Mol Biol 2016;51(3):162-84.

73. Forester CM, Maddox J, Louis JV, Goris J, Virshup DM. Control of mitotic exit by PP2A regulation of $\mathrm{Cdc} 25 \mathrm{C}$ and Cdk1. Proceedings of the National Academy of Sciences of the United States of America 2007;104(50):19867-72.

74. Riedel CG, Katis VL, Katou Y, Mori S, Itoh T, Helmhart W, et al. Protein phosphatase $2 \mathrm{~A}$ protects centromeric sister chromatid cohesion during meiosis I. Nature 2006;441(7089):53-61.

75. Kitajima TS, Sakuno T, Ishiguro K, lemura S, Natsume T, Kawashima SA, et al. Shugoshin collaborates with protein phosphatase $2 \mathrm{~A}$ to protect cohesin. Nature 2006;441(7089):46-52.

76. Vigneron S, Brioudes E, Burgess A, Labbe JC, Lorca T, Castro A. Greatwall maintains mitosis through regulation of PP2A. The EMBO journal 2009;28(18):2786-93.

77. Lorca T, Bernis C, Vigneron S, Burgess A, Brioudes E, Labbe JC, et al. Constant regulation of both the MPF amplification loop and the Greatwall-PP2A pathway is required for metaphase II arrest and correct entry into the first embryonic cell cycle. J Cell Sci 2010;123(Pt 13):2281-91.

78. Joukov V, De Nicolo A. Aurora-PLK1 cascades as key signaling modules in the regulation of mitosis. Sci Signal 2018;11(543). 
79. Kim SY, Hyun SY, Jang YJ. Dephosphorylation of Plk1 occurs through PP2AB55/ENSA/Greatwall pathway during mitotic DNA damage recovery. Cell Cycle 2019;18(10):1154-67.

80. Feng J, Wakeman T, Yong S, Wu X, Kornbluth S, Wang XF. Protein phosphatase $2 A$-dependent dephosphorylation of replication protein $A$ is required for the repair of DNA breaks induced by replication stress. Molecular and cellular biology 2009;29(21):5696-709.

81. Wang Q, Gao F, Wang T, Flagg T, Deng X. A nonhomologous end-joining pathway is required for protein phosphatase 2A promotion of DNA double-strand break repair. Neoplasia 2009;11(10):1012-21.

82. Douglas P, Moorhead GB, Ye R, Lees-Miller SP. Protein phosphatases regulate DNA-dependent protein kinase activity. J Biol Chem 2001;276(22):18992-8.

83. Hawkins AJ, Subler MA, Akopiants K, Wiley JL, Taylor SM, Rice AC, et al. In vitro complementation of Tdp1 deficiency indicates a stabilized enzyme-DNA adduct from tyrosyl but not glycolate lesions as a consequence of the SCAN1 mutation. DNA Repair (Amst) 2009;8(5):654-63.

84. Bunting SF, Callen E, Wong N, Chen HT, Polato F, Gunn A, et al. 53BP1 inhibits homologous recombination in Brca1-deficient cells by blocking resection of DNA breaks. Cell 2010;141(2):243-54.

85. Kalev P, Simicek M, Vazquez I, Munck S, Chen L, Soin T, et al. Loss of PPP2R2A inhibits homologous recombination DNA repair and predicts tumor sensitivity to PARP inhibition. Cancer research 2012;72(24):6414-24.

86. Chen CC, Feng W, Lim PX, Kass EM, Jasin M. Homology-Directed Repair and the Role of BRCA1, BRCA2, and Related Proteins in Genome Integrity and Cancer. Annu Rev Cancer Biol 2018;2:313-36.

87. Ceccaldi R, Rondinelli B, D'Andrea AD. Repair Pathway Choices and Consequences at the Double-Strand Break. Trends Cell Biol 2016;26(1):52-64.

88. Ghezraoui H, Piganeau M, Renouf B, Renaud JB, Sallmyr A, Ruis B, et al. Chromosomal translocations in human cells are generated by canonical nonhomologous end-joining. Mol Cell 2014;55(6):829-42.

89. Zhang $\mathrm{Y}$, Jasin $\mathrm{M}$. An essential role for CtIP in chromosomal translocation formation through an alternative end-joining pathway. Nature structural \& molecular biology 2011;18(1):80-4.

90. Simsek D, Brunet E, Wong SY, Katyal S, Gao Y, McKinnon PJ, et al. DNA ligase III promotes alternative nonhomologous end-joining during chromosomal translocation formation. PLoS Genet 2011;7(6):e1002080.

91. Simsek D, Jasin M. Alternative end-joining is suppressed by the canonical NHEJ component Xrcc4-ligase IV during chromosomal translocation formation. Nature structural \& molecular biology 2010;17(4):410-6.

92. Sallmyr A, Tomkinson AE. Repair of DNA double-strand breaks by mammalian alternative end-joining pathways. J Biol Chem 2018;293(27):10536-46.

93. Kudo N, Matsumori N, Taoka H, Fujiwara D, Schreiner EP, Wolff B, et al. Leptomycin $B$ inactivates CRM1/exportin 1 by covalent modification at a cysteine residue in the central conserved region. Proc Natl Acad Sci U S A 1999;96(16):9112-7. 
94. Cai X, Liu X. Inhibition of Thr-55 phosphorylation restores p53 nuclear localization and sensitizes cancer cells to DNA damage. Proc Natl Acad Sci U S A 2008;105(44):16958-63.

95. Feng Z, Kachnic L, Zhang J, Powell SN, Xia F. DNA damage induces p53dependent BRCA1 nuclear export. The Journal of biological chemistry 2004;279(27):28574-84.

96. Han X, Saito H, Miki Y, Nakanishi A. A CRM1-mediated nuclear export signal governs cytoplasmic localization of BRCA2 and is essential for centrosomal localization of BRCA2. Oncogene 2008;27(21):2969-77.

97. la Cour T, Kiemer L, Molgaard A, Gupta R, Skriver K, Brunak S. Analysis and prediction of leucine-rich nuclear export signals. Protein Eng Des Sel 2004;17(6):527-36.

98. Fornerod M, Ohno M, Yoshida M, Mattaj IW. CRM1 is an export receptor for leucine-rich nuclear export signals. Cell 1997;90(6):1051-60.

99. Fukuda M, Asano S, Nakamura T, Adachi M, Yoshida M, Yanagida M, et al. CRM1 is responsible for intracellular transport mediated by the nuclear export signal. Nature 1997;390(6657):308-11.

100. Ossareh-Nazari B, Gwizdek C, Dargemont C. Protein export from the nucleus. Traffic 2001;2(10):684-9.

101. Guttler T, Gorlich D. Ran-dependent nuclear export mediators: a structural perspective. The EMBO journal 2011;30(17):3457-74.

102. Klebe $\mathrm{C}$, Bischoff FR, Ponstingl H, Wittinghofer A. Interaction of the nuclear GTP-binding protein Ran with its regulatory proteins RCC1 and RanGAP1. Biochemistry 1995;34(2):639-47.

103. Kellogg GE, Semus SF, Abraham DJ. HINT: a new method of empirical hydrophobic field calculation for CoMFA. J Comput Aided Mol Des 1991;5(6):545-52.

104. Marabotti A, Balestreri L, Cozzini P, Mozzarelli A, Kellogg GE, Abraham DJ. HINT predictive analysis of binding between retinol binding protein and hydrophobic ligands. Bioorg Med Chem Lett 2000;10(18):2129-32.

105. Monecke T, Guttler T, Neumann P, Dickmanns A, Gorlich D, Ficner R. Crystal structure of the nuclear export receptor CRM1 in complex with Snurportin1 and RanGTP. Science 2009;324(5930):1087-91.

106. Grinthal A, Adamovic I, Weiner B, Karplus M, Kleckner N. PR65, the HEATrepeat scaffold of phosphatase PP2A, is an elastic connector that links force and catalysis. Proc Natl Acad Sci U S A 2010;107(6):2467-72.

107. Andjelkovic M, Jakubowicz T, Cron P, Ming XF, Han JW, Hemmings BA. Activation and phosphorylation of a pleckstrin homology domain containing protein kinase (RAC-PK/PKB) promoted by serum and protein phosphatase inhibitors. Proc Natl Acad Sci U S A 1996;93(12):5699-704.

108. Sablina AA, Hector M, Colpaert N, Hahn WC. Identification of PP2A complexes and pathways involved in cell transformation. Cancer research 2010;70(24):10474-84.

109. Perry J, Kleckner N. The ATRs, ATMs, and TORs are giant HEAT repeat proteins. Cell 2003;112(2):151-5. 
110. Petosa C, Schoehn G, Askjaer P, Bauer U, Moulin M, Steuerwald U, et al. Architecture of CRM1/Exportin1 suggests how cooperativity is achieved during formation of a nuclear export complex. Mol Cell 2004;16(5):761-75.

111. Lee DH, Chowdhury D. What goes on must come off: phosphatases gate-crash the DNA damage response. Trends Biochem Sci 2011;36(11):569-77.

112. Viniegra JG, Martinez N, Modirassari P, Losa JH, Parada Cobo C, Lobo VJ, et al. Full activation of $\mathrm{PKB} / \mathrm{Akt}$ in response to insulin or ionizing radiation is mediated through ATM. J Biol Chem 2005;280(6):4029-36.

113. Rothkamm K, Kruger I, Thompson LH, Lobrich M. Pathways of DNA doublestrand break repair during the mammalian cell cycle. Mol Cell Biol 2003;23(16):5706-15.

114. Heinrich R, Neel BG, Rapoport TA. Mathematical models of protein kinase signal transduction. Mol Cell 2002;9(5):957-70. 
Firiurath Figure 7.

A

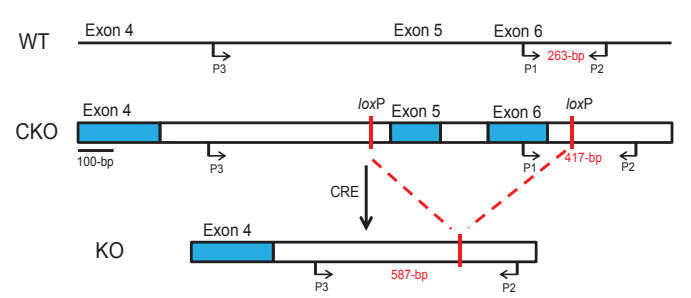

D

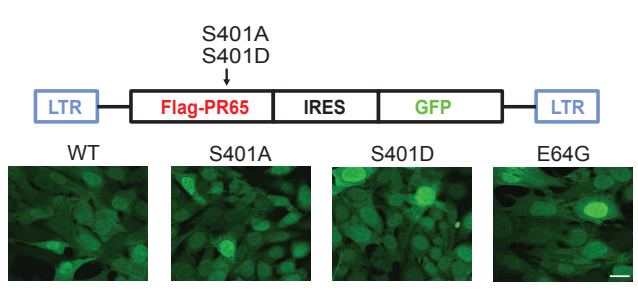

B
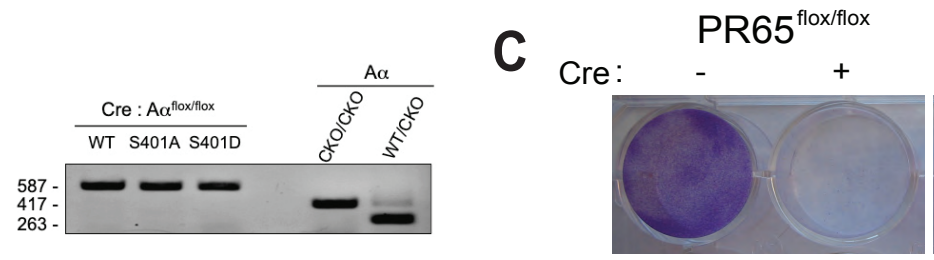

PR65 $5^{\text {flox/E64G }}$ $+$

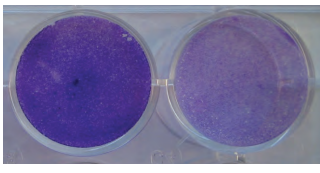

E
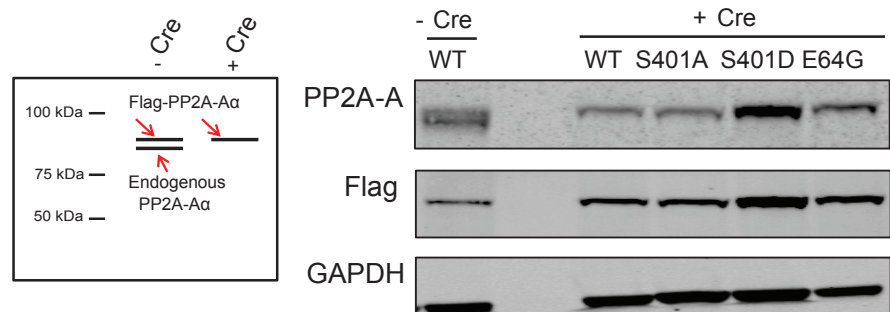


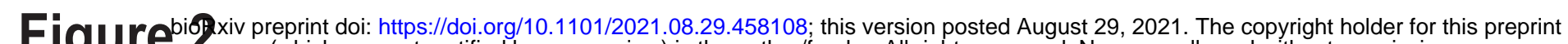
(which was not certified by peer review) is the author/funder. All rights reserved. No reuse allowed without permission.

A
$\stackrel{0}{a}$
$\ddot{0}$
$\frac{\pi}{4}$

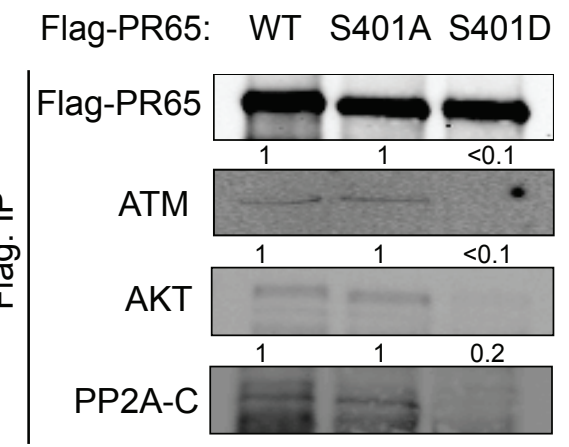

B
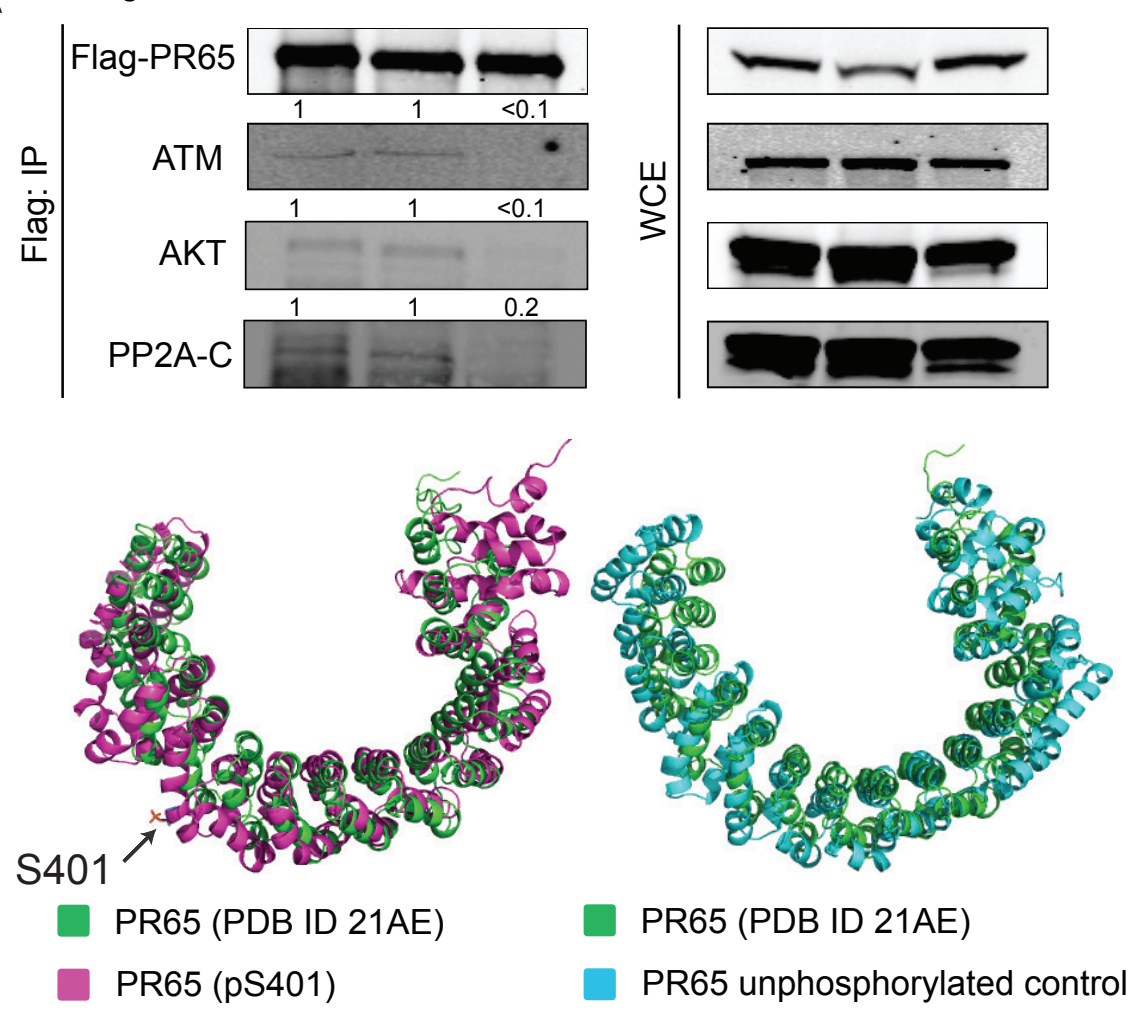

C
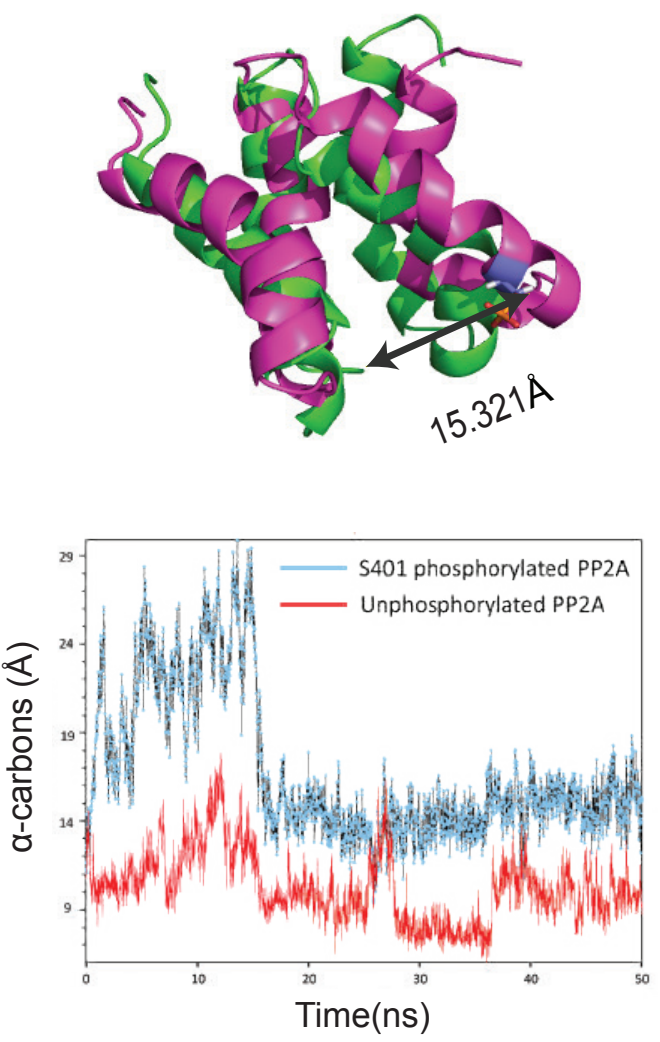
Fi Figure

\section{A}

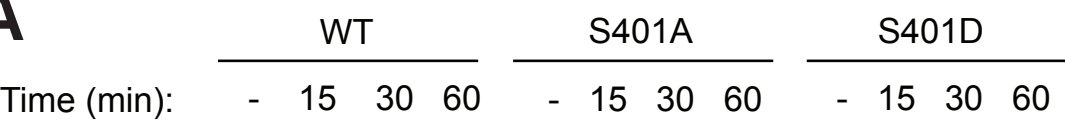

p-AKT

AKT

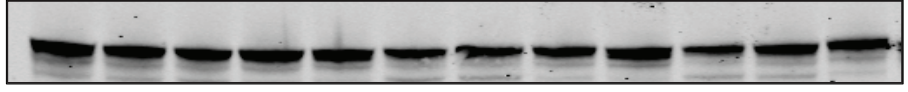

GAPDH

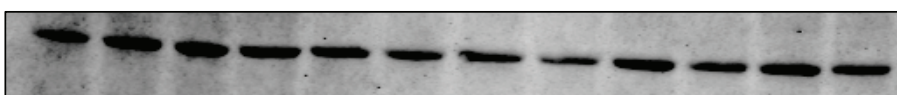

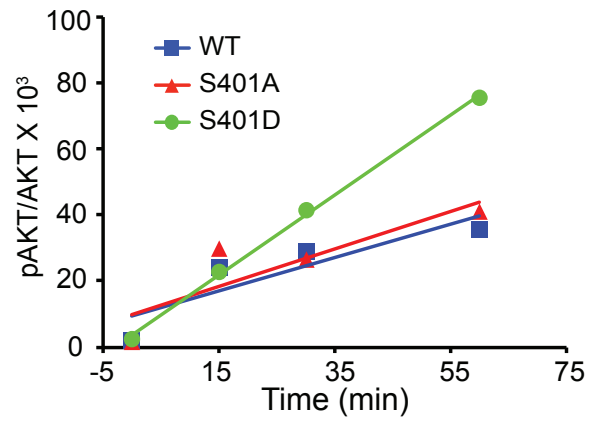

B

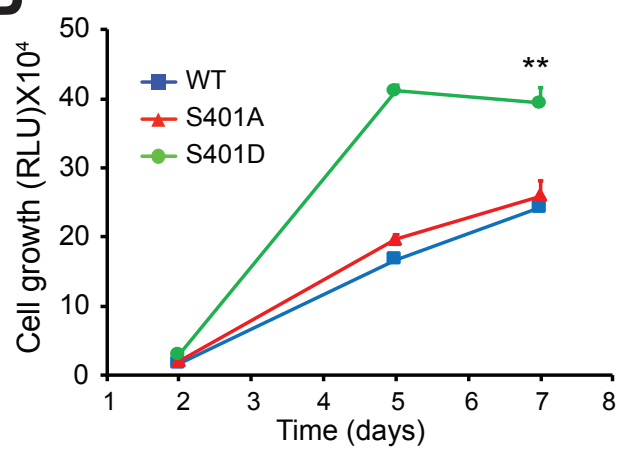

C

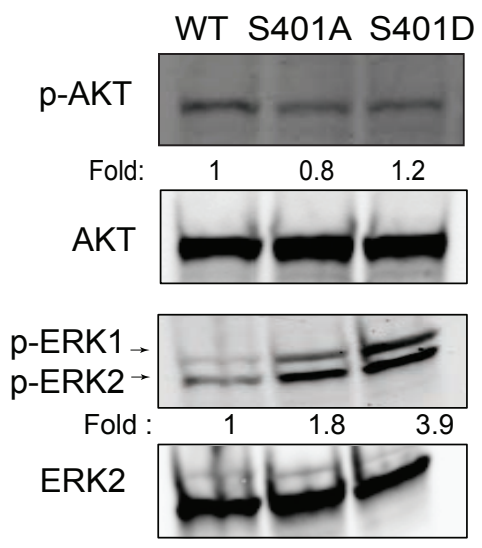

D
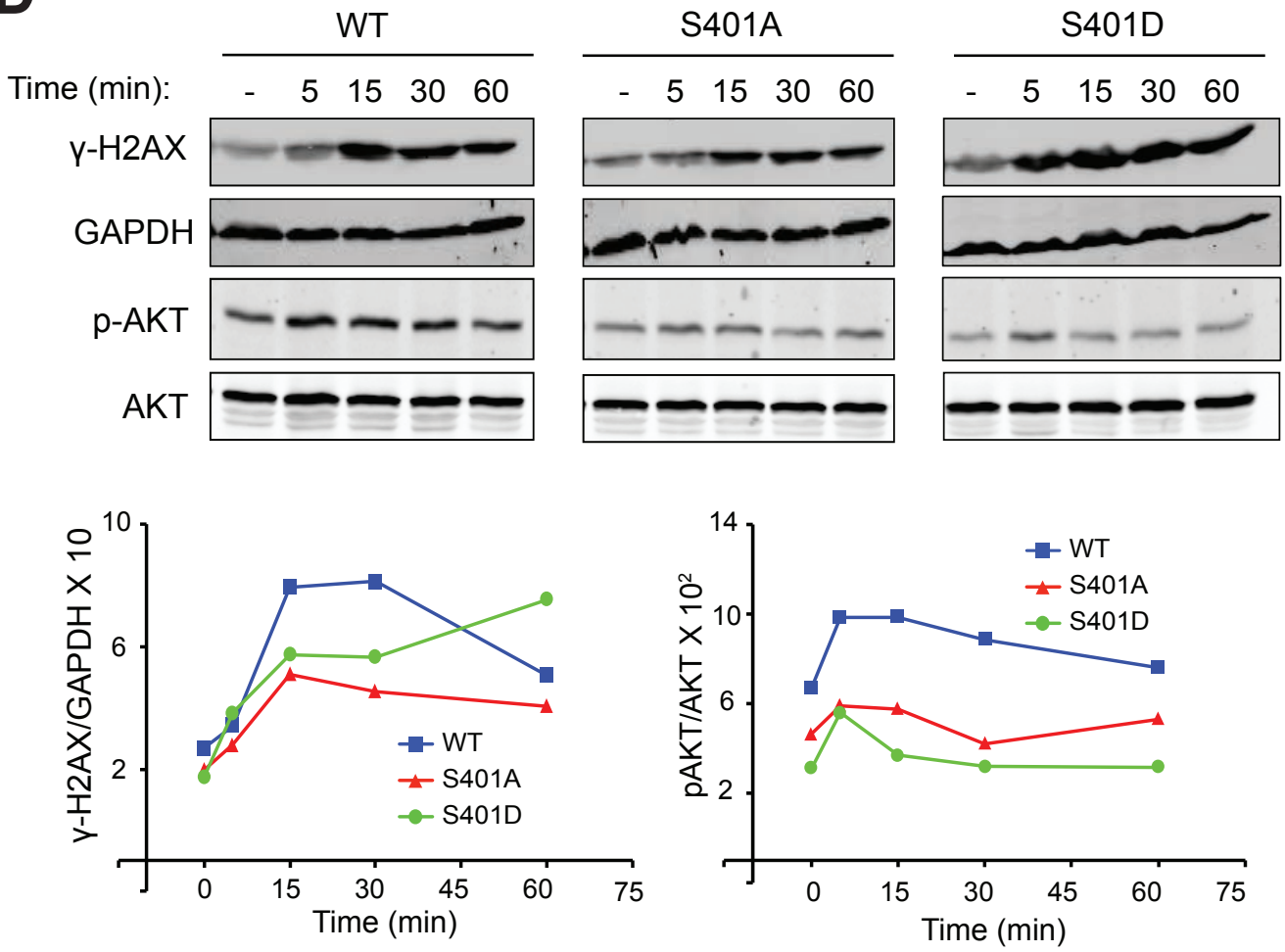


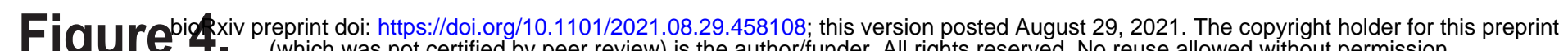
Figure" 4.

A
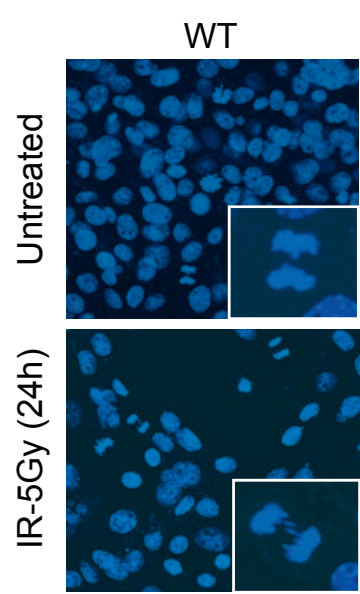

D
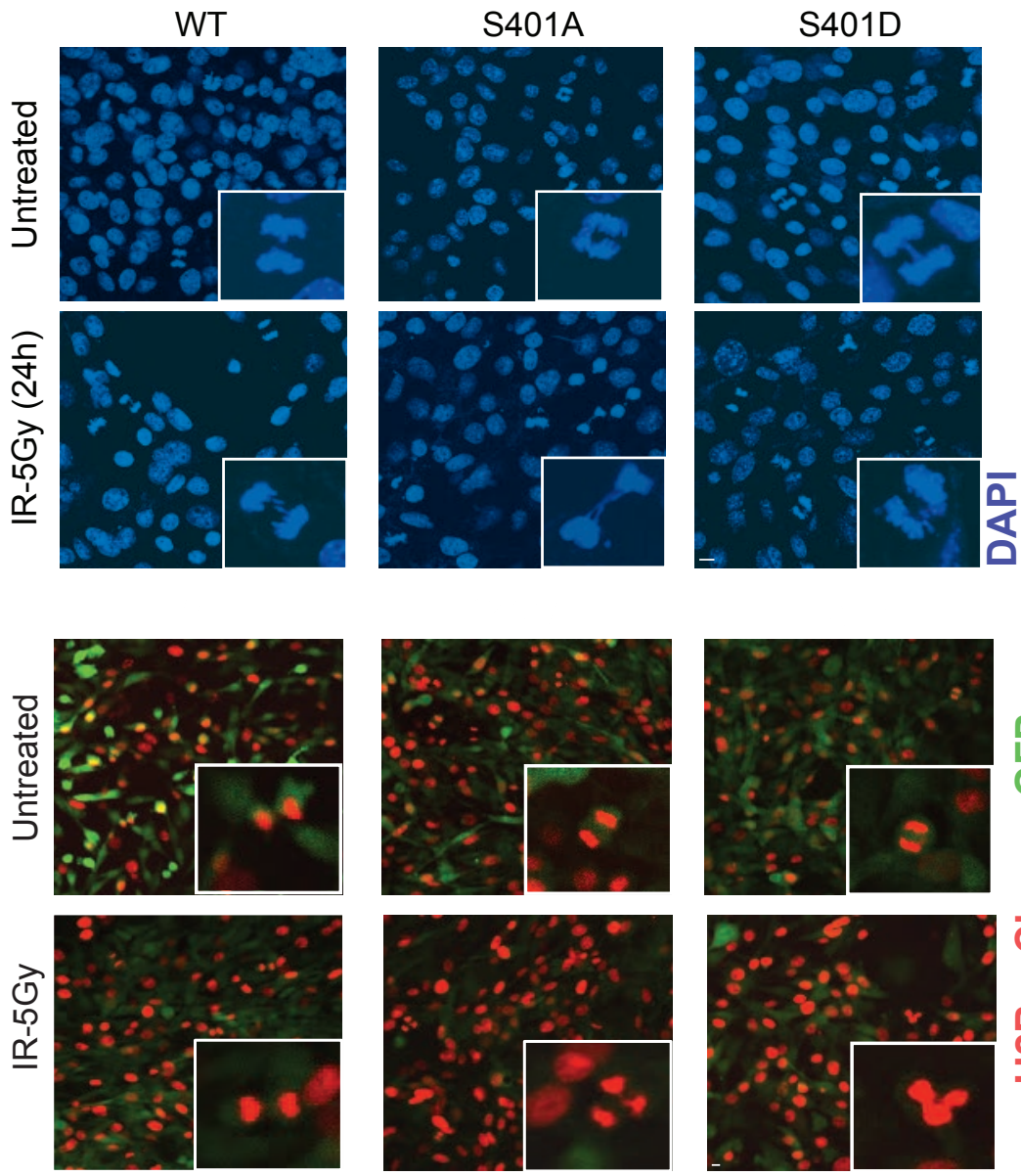

C
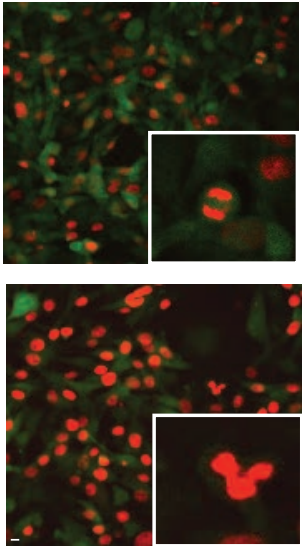

B

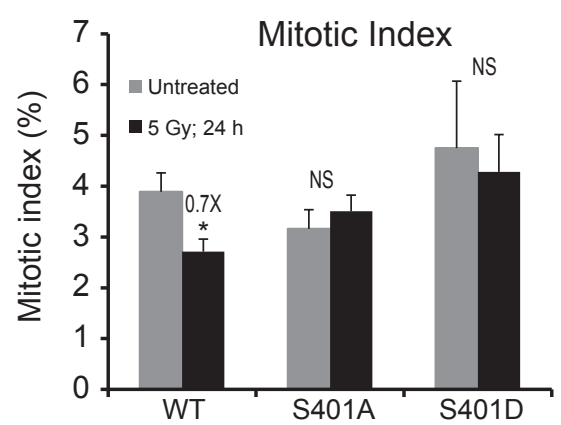

o

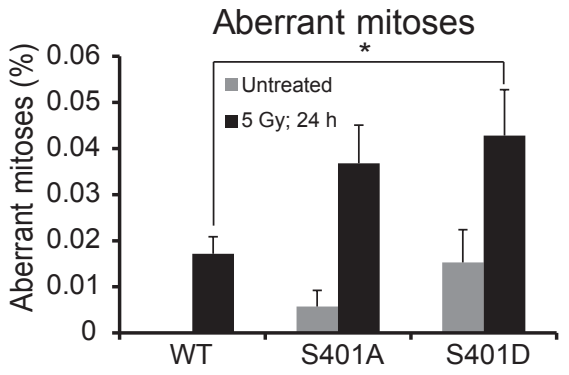

Mitotic length

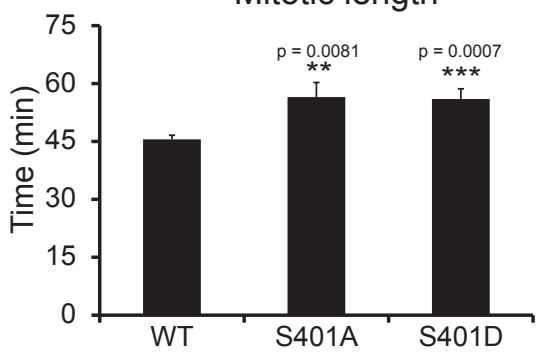

G

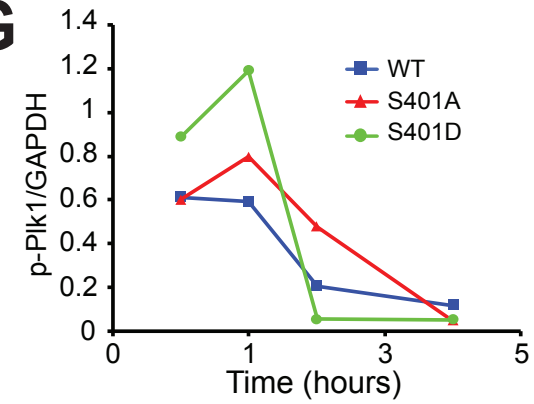

H
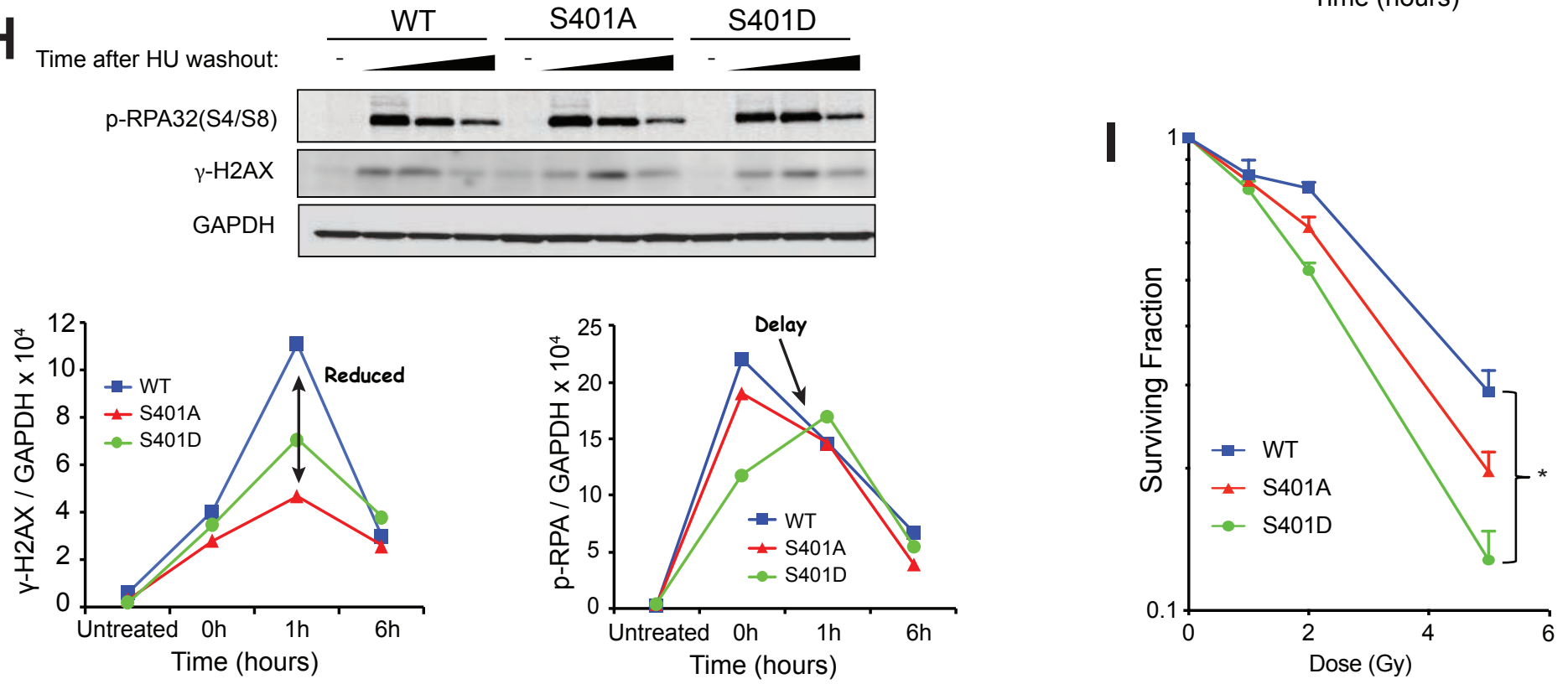


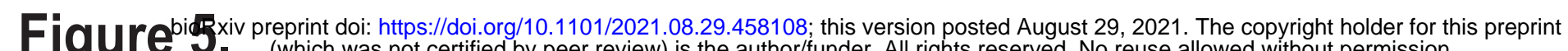

A

NHEJ Assay

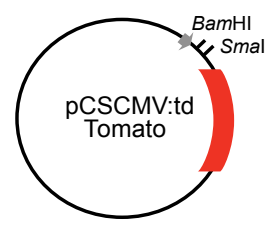

$\downarrow \begin{gathered}B \text { digest } \\ \text { BaI }+ \text { Sma }\end{gathered}$

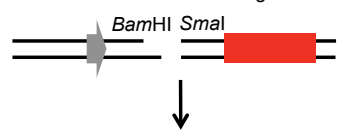

Linearized pCSCMV:tdTomato

$\downarrow$ Transfect

MEFs (WT/S401A/S401D)

Count RFP ${ }^{+}$cells/PCR Assay
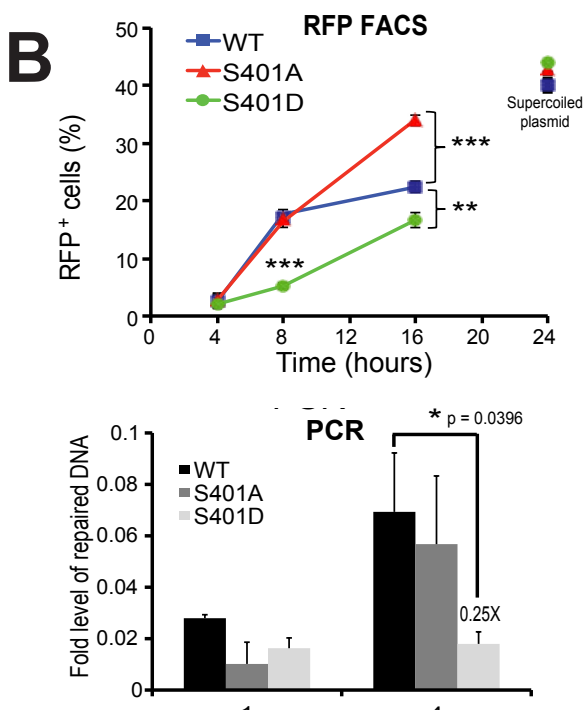

1 Time (hours) ${ }^{4}$

E
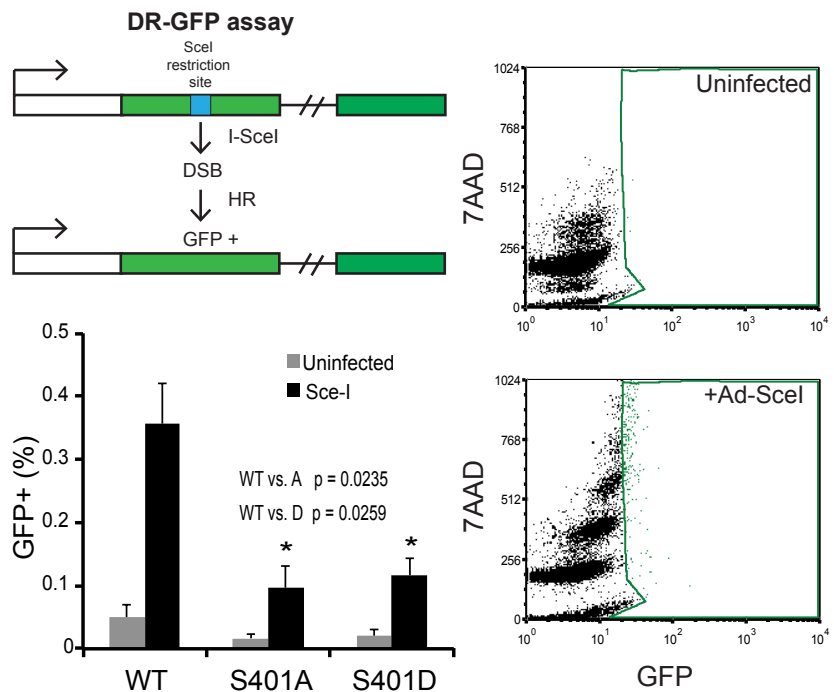

$\uparrow$ Ligation

$\uparrow$ Gap filling ( $\mathrm{T}$ )

$\uparrow$ Annealing

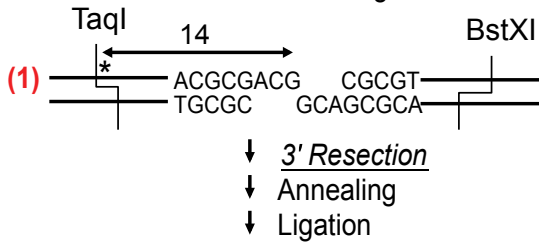

(3)

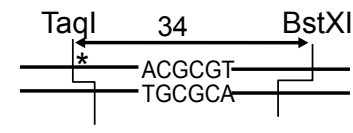

$D$ IR:

Time
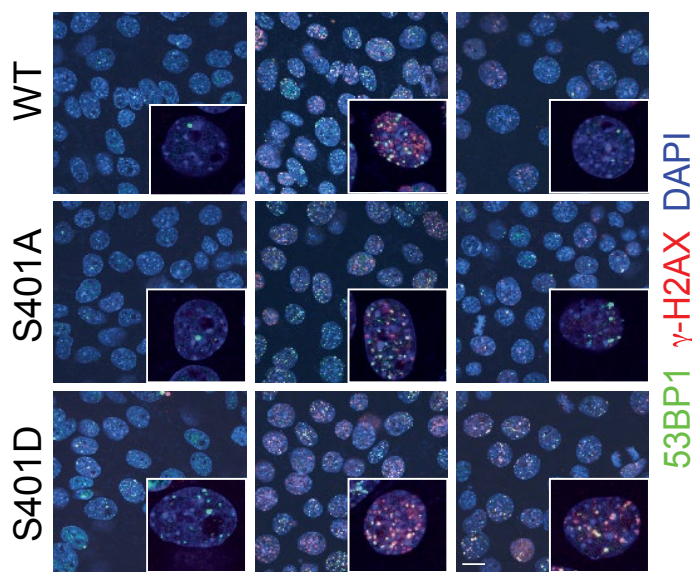

$\mathbf{F}$
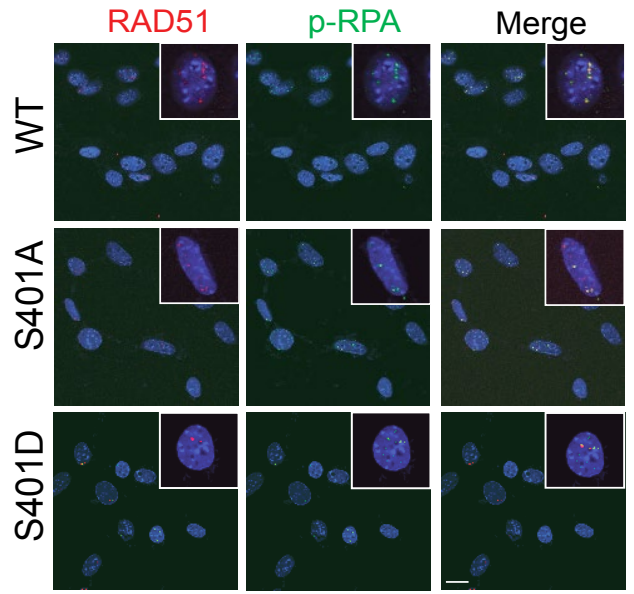
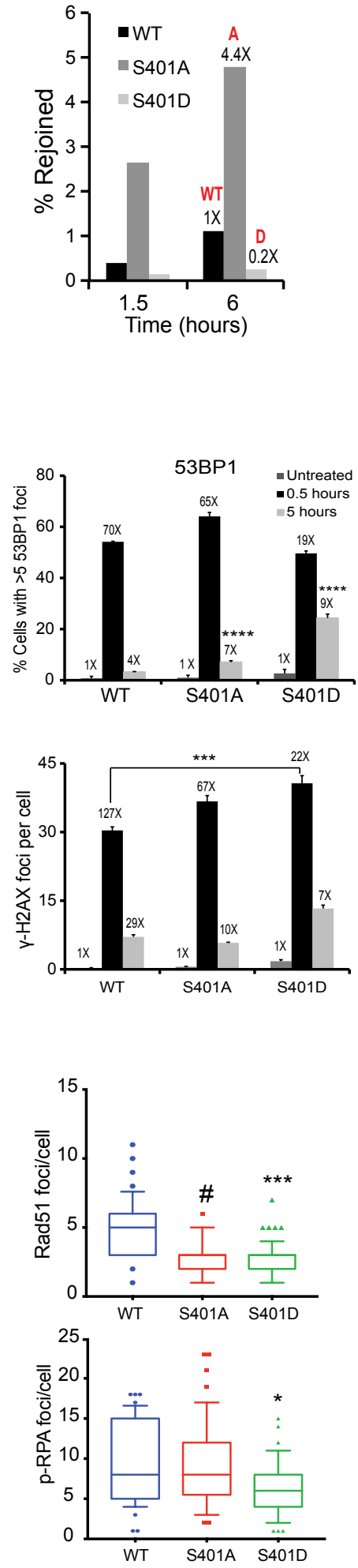

Figure 6.
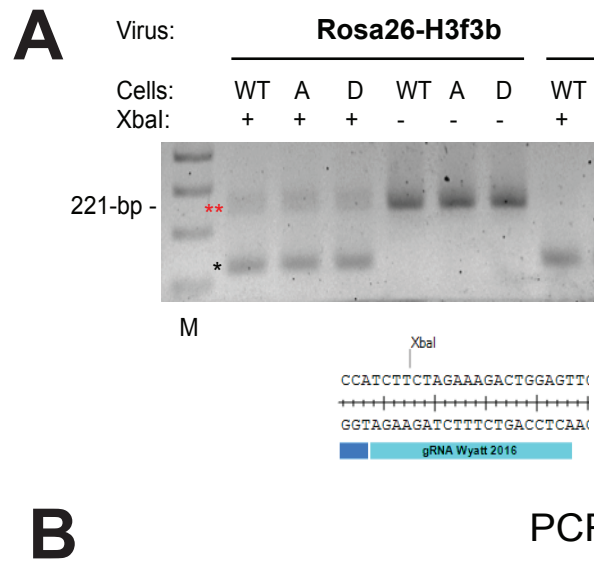

B

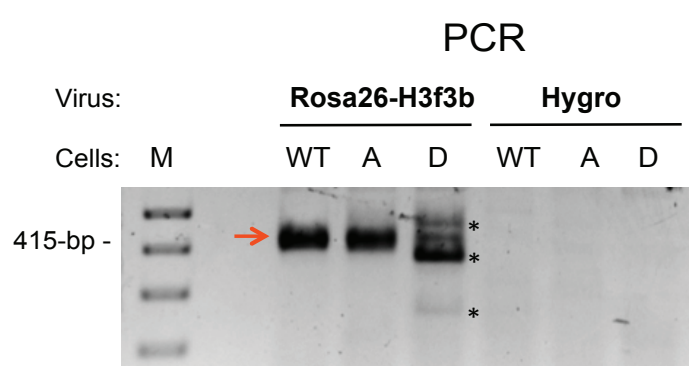

pGEM-T Easy

Virus: Rosa26-H3f3b Cells: M WT A D

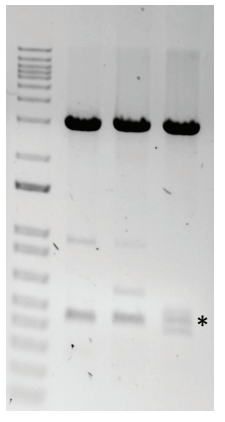

EcoRI digests
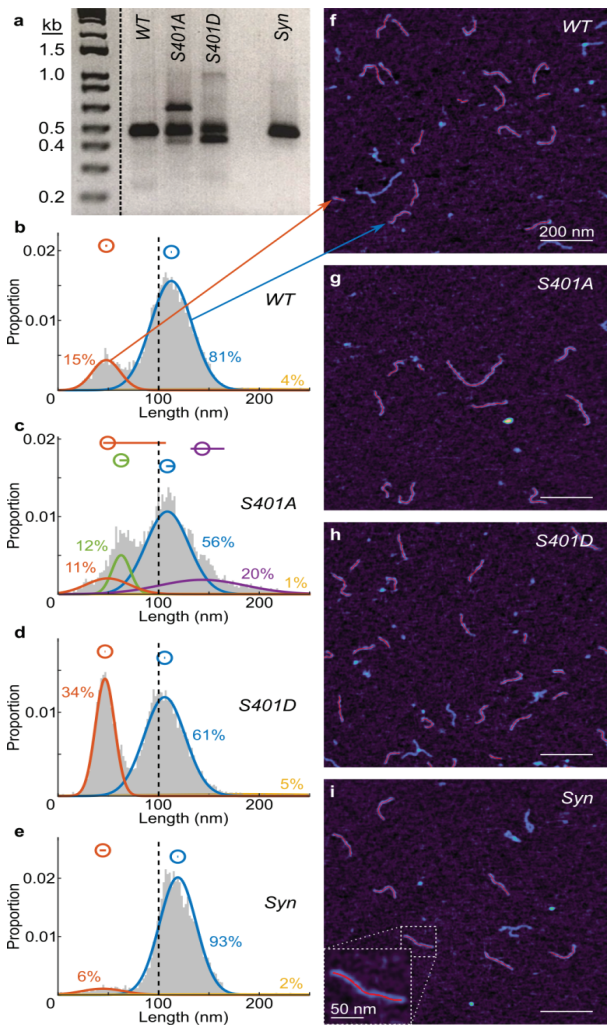
Figure A

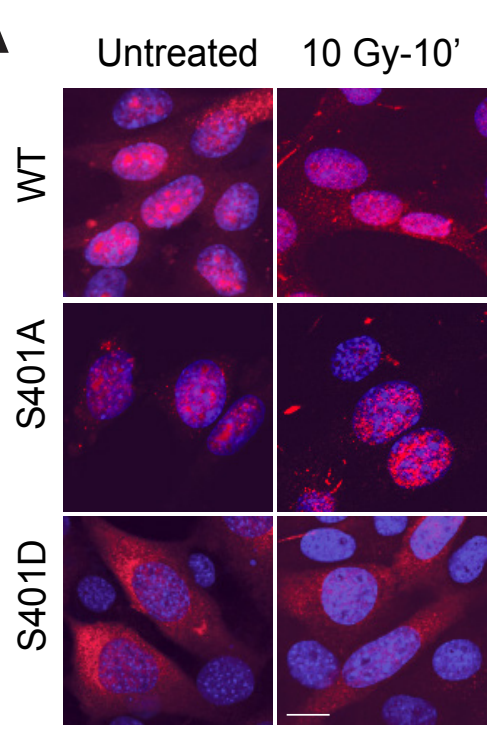

FLAGDAPI

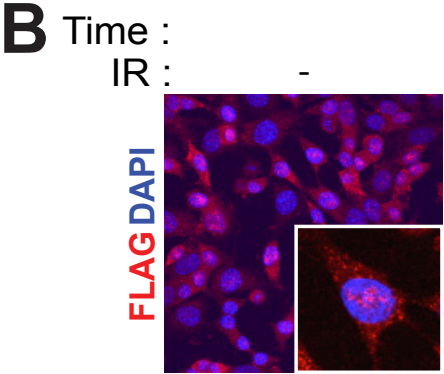

LMB:

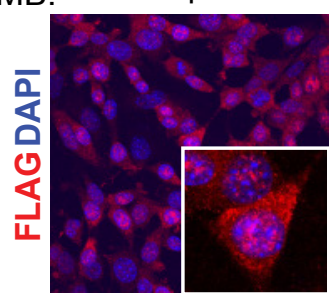

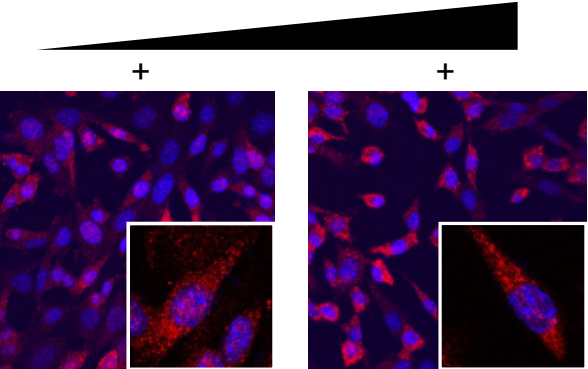

$+$
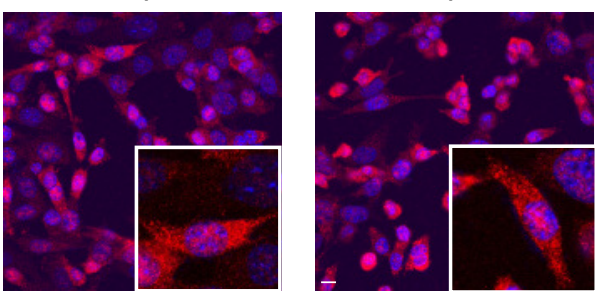

LMB (Leptomycin-B)-CRM1 inhibitor
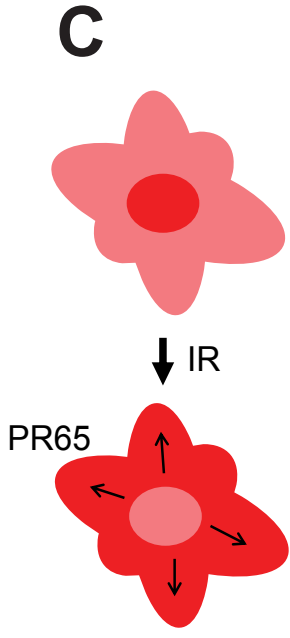


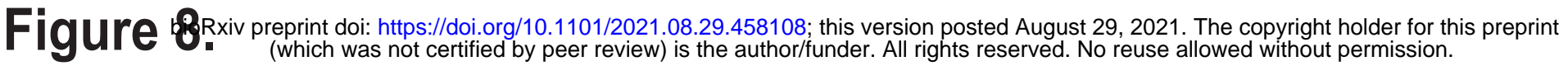

A

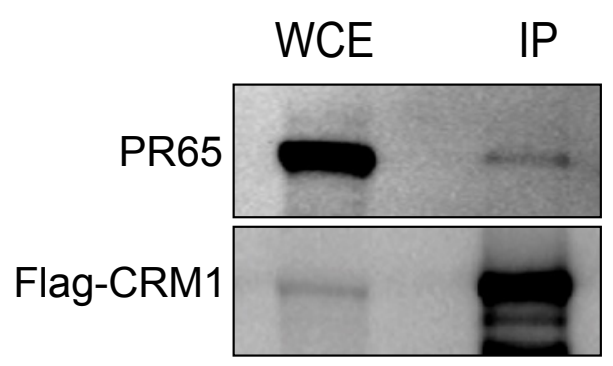

C

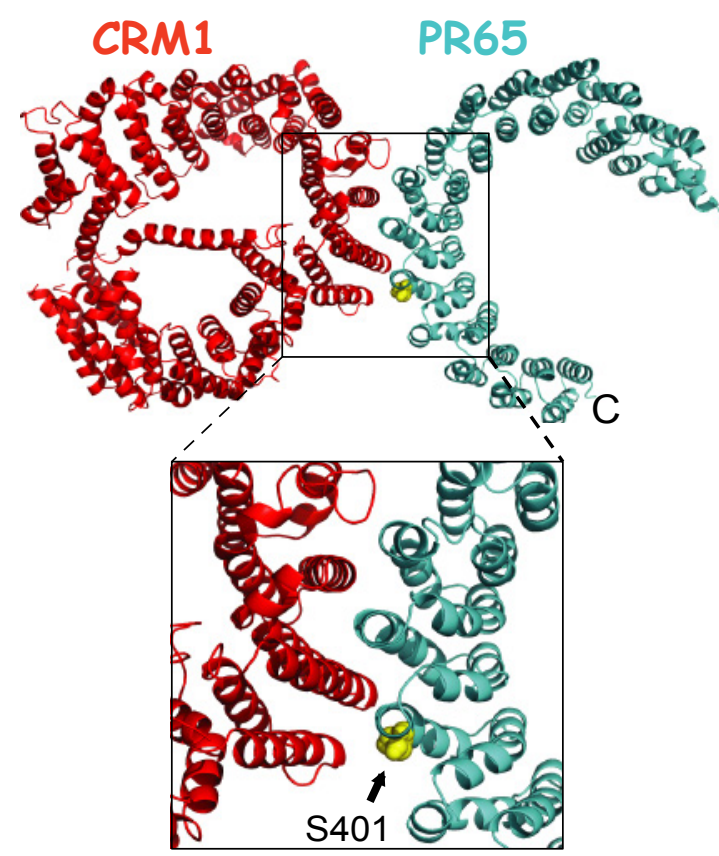

B
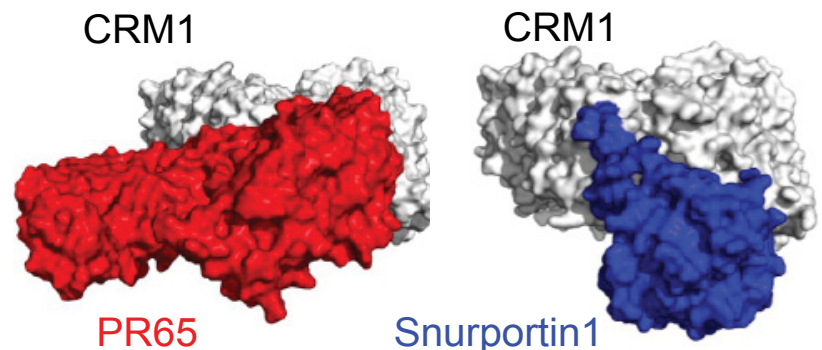

HINT scores:

CRM1/WT:

CRM1/S401A:

CRM1/S401D:

5372

5211

CRM1/Snurportin1: 1321

Interface areas (Angstrom²):

CRM1/PP2A :

1348

CRM1/Snurportin1: 1177
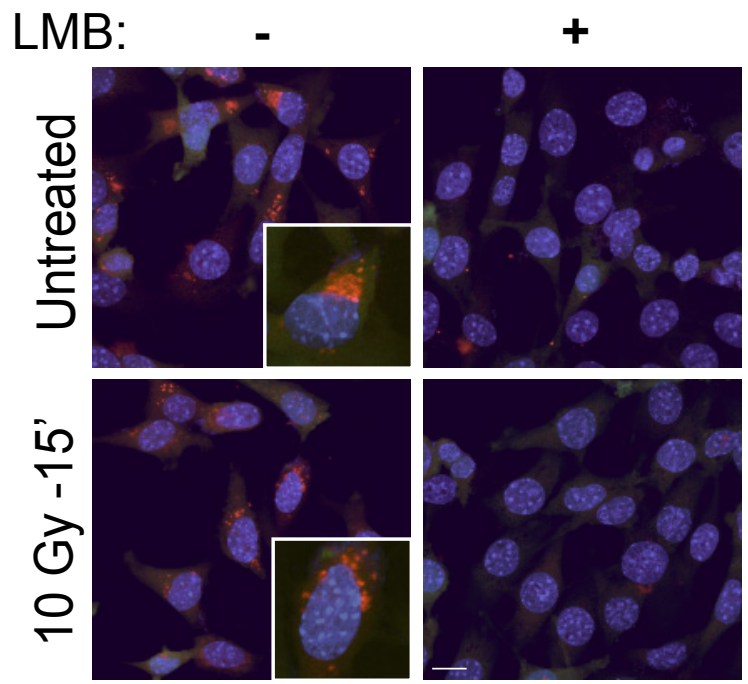

FIg Ure b. Bxiv preprint doi: https://doi.org/10.1101/2021.08.29.458108; this version posted August 29, 2021. The copyright holder for this preprint
(which was not certified by peer review) is the author/funder. All rights reserved. No reuse allowed without permission.

A

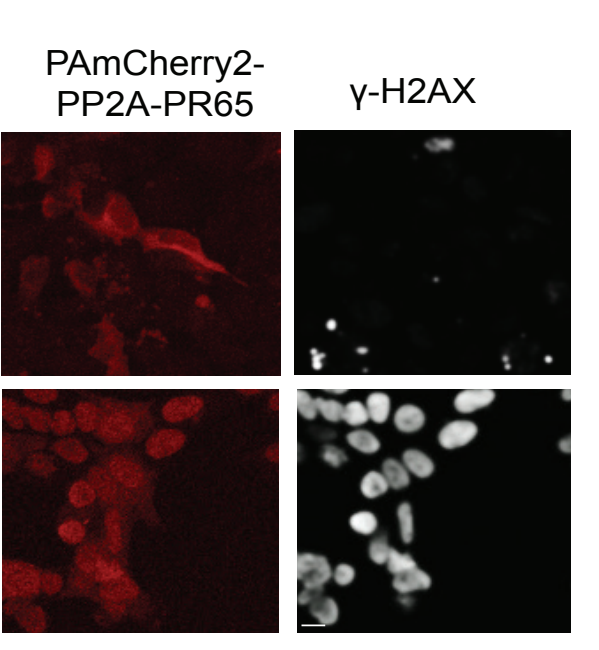

B

Hoechst

33258

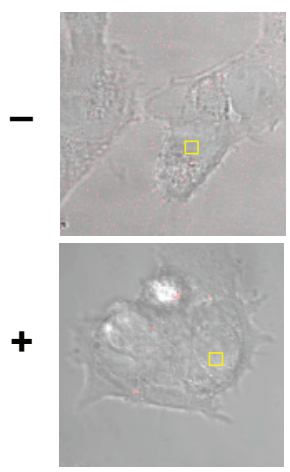

Time after laser (405 nm)

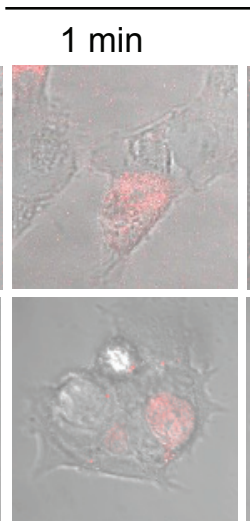

$4 \mathrm{~min}$

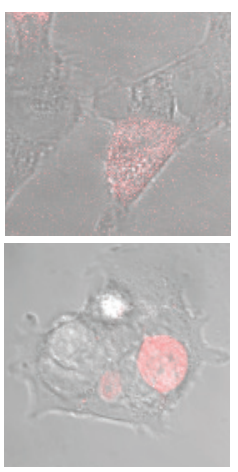

$7 \mathrm{~min}$

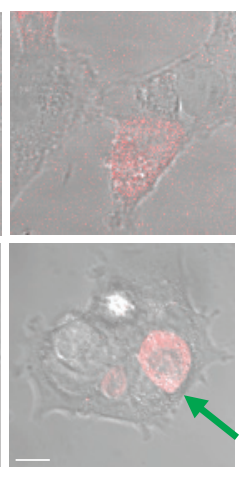

C

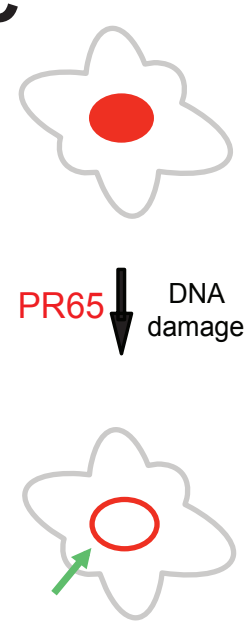

D
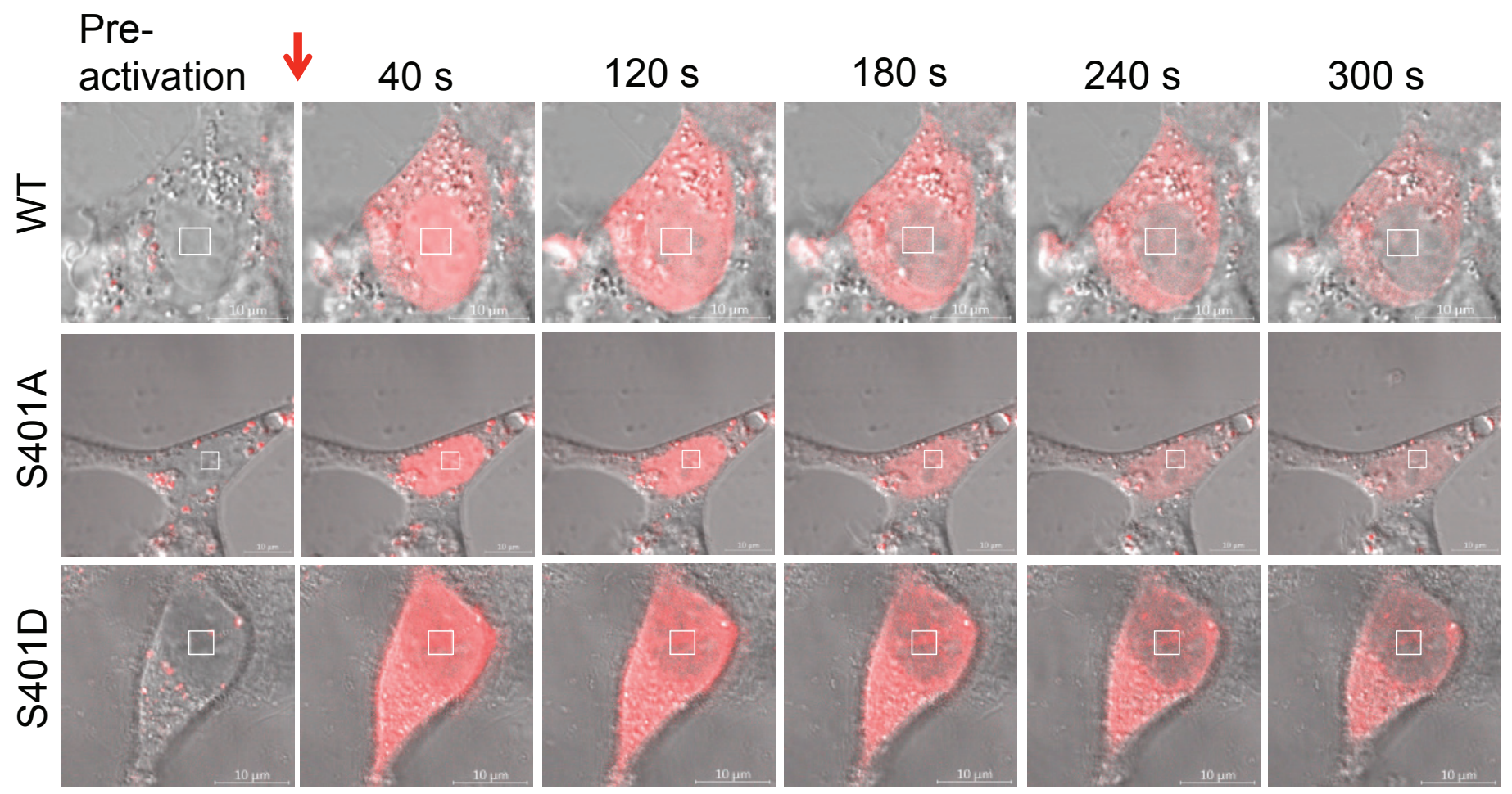

$\mathbf{E}$
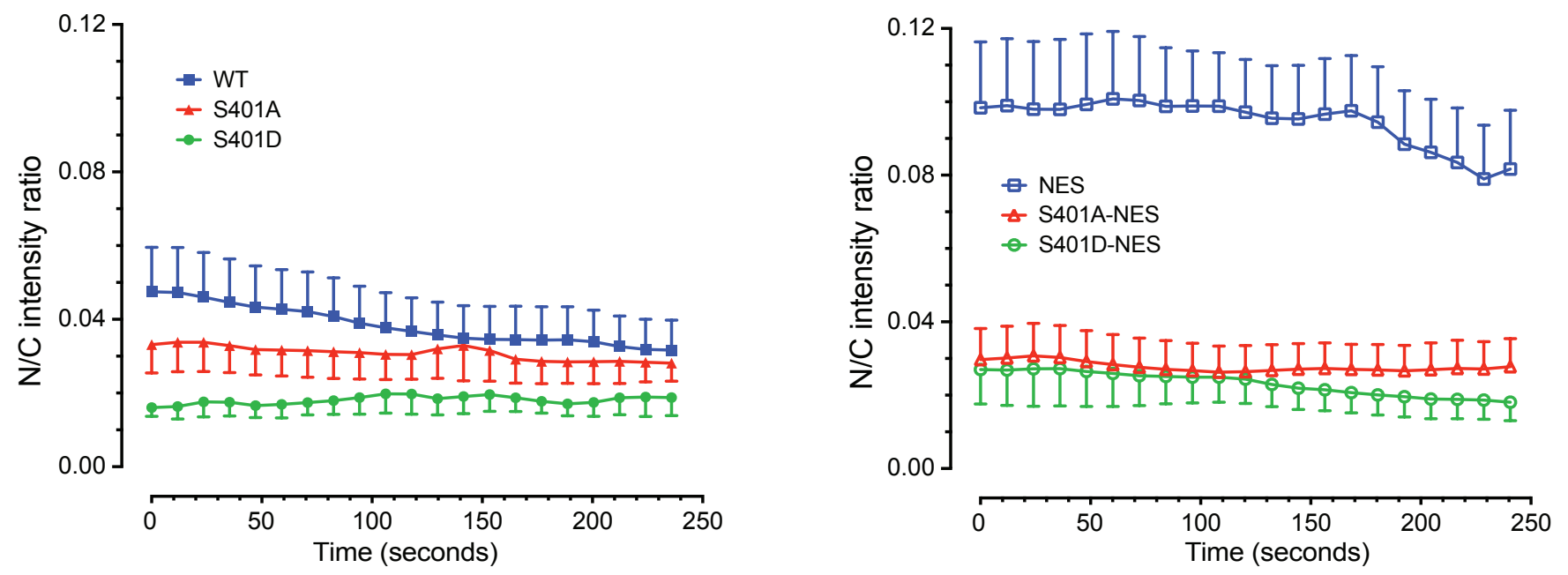
EIOUTe brffy preprint doi: https://doi.org/10.1101/2021.08.29.458108; this version posted August 29, 2021. The copyright holder for this preprint (which was not certified by peer review) is the author/funder. All rights reserved. No reuse allowed without permission.

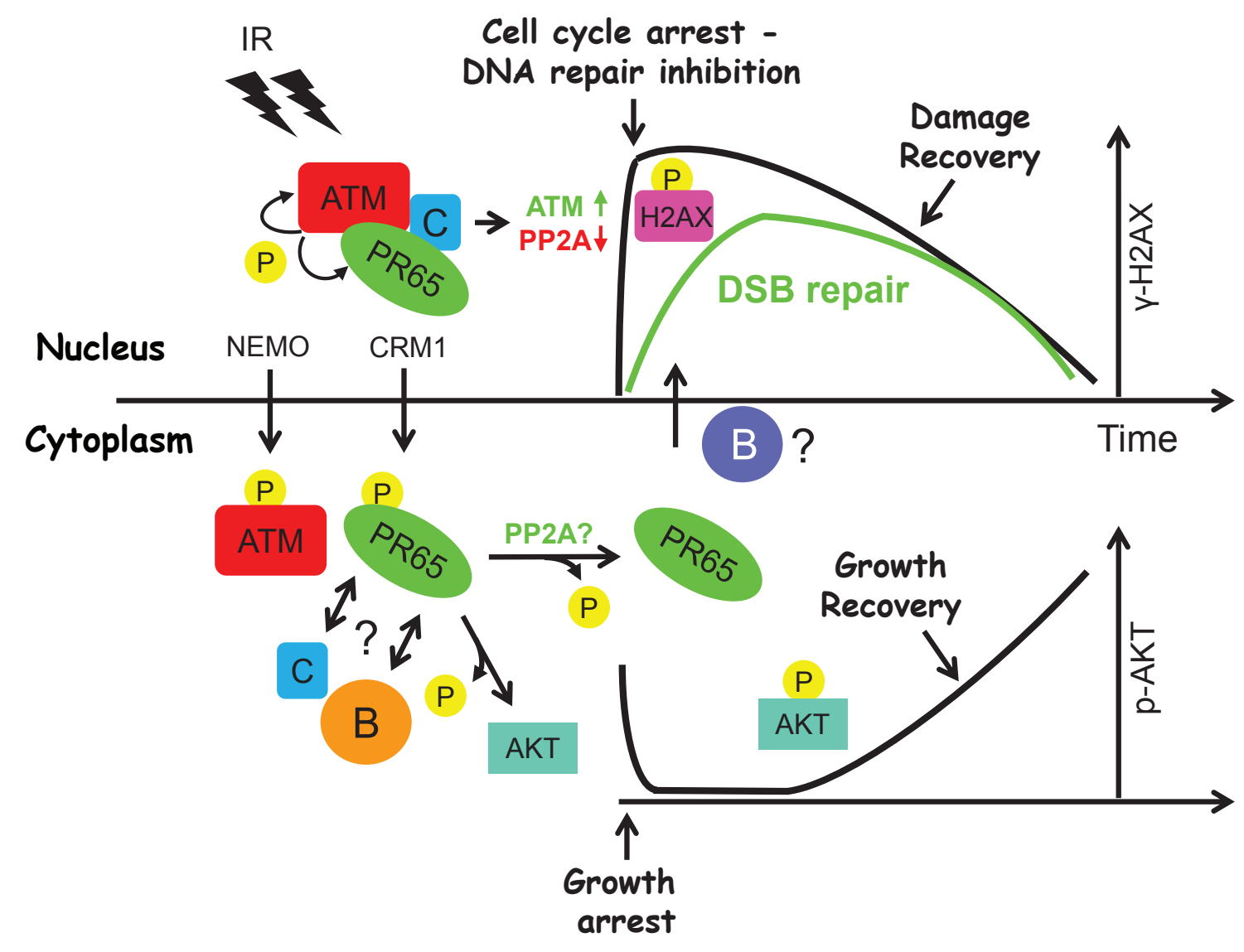




\section{Supplemental data}

\section{ATM phosphorylates PP2A subunit A resulting in nuclear export and spatiotemporal regulation of the DNA damage response}

Amrita Sule ${ }^{1,2}$, Sarah E. Golding ${ }^{1}$, Syed F. Farhan ${ }^{1,2}$, James Watson ${ }^{1}$, Mostafa H. Ahmed $^{3}$, Glen E. Kellogg ${ }^{3,7}$, Tytus Bernas ${ }^{4}$, Sean Koebley ${ }^{5}$, Jason C. Reed ${ }^{5,7}$, Lawrence F. Povirk ${ }^{6,7}$, and Kristoffer Valerie ${ }^{1,2,7}$,

Departments of ${ }^{1}$ Radiation Oncology, ${ }^{2}$ Biochemistry and Molecular Biology, ${ }^{3}$ Medicinal Chemistry, ${ }^{4}$ Anatomy, ${ }^{5}$ Physics, ${ }^{6}$ Pharmacology and Toxicology, and the ${ }^{7}$ Massey Cancer Center,

Virginia Commonwealth University, Richmond, VA 23298, USA 


\section{Materials and Methods:}

\section{Plasmids:}

\begin{tabular}{|l|l|l|}
\hline Plasmids & Source & Provided by \\
\hline pMIG-Aalpha & Addgene \#10884 & William Hahn \\
\hline Flag-hCRM1 & Addgene \#17647 & Xin Wang \\
\hline pmCherry-C1-RanQ69L & Addgene \#30309 & Jay Brenman \\
\hline pCSCMV:tdTomato & Addgene \#30530 & Gerhart Ryffel \\
\hline $\begin{array}{l}\text { Human TERT (LOX-TERT- } \\
\text { iresTK }\end{array}$ & Addgene \#12245 & Didier Trono \\
\hline pcDNA3-mRuby2 & Addgene \#40260 & Michael Lin \\
\hline pGEX-2T & GE Life Sciences \#28954653 & \\
\hline YFP-ATM & & David Chen \\
\hline pBabe-hygroPyMT & Addgene \#22305 & Filippo Giancotti \\
\hline pPAmCherry-a-tubulin & Addgene \#31930 & Vladislav \\
\hline LentiCRISPR v2 & Addgene plasmid \# 52961 & Feng Zhang \\
\hline LentiCRISPRv2 hygro & Addgene plasmid \# 98291 & Brett Stringer \\
\hline pCEP-4HA-B56 alpha & Addgene \#14532 & David Virshup \\
\hline pCEP-4HA-B56 gamma3 & Addgene \#14535 & David Virshup \\
\hline pCEP-4HA-B56 gamma1 & Addgene \#14534 & David Virshup \\
\hline pCEP-4HA-B56 epsilon & Addgene \#14537 & David Virshup \\
\hline pCEP-4HA-B56 delta & Addgene \#14536 & David Virshup \\
\hline pCEP-4HA-B56 beta & Addgene \#14533 & David Virshup \\
\hline & $\begin{array}{l}\text { Created by replacing B56 alpha } \\
\text { from pCEP-4HA-B56 beta with B55 } \\
\text { alpha. The B55 alpha cDNA was } \\
\text { optained from pUC19-hPPP2R2A by } \\
\text { PCR. }\end{array}$ & This study \\
\hline pCEP-4HA-B55 alpha & & \\
\hline
\end{tabular}




\section{Antibodies and Reagents:}

\begin{tabular}{|l|l|l|}
\hline \multicolumn{1}{|c|}{ Antibodies and Reagents } & \multicolumn{1}{c|}{ Company } & \multicolumn{1}{c|}{ Catalog no. } \\
\hline anti-ATM & Cell Signaling Technology & 2873 \\
\hline anti-AKT & Cell Signaling Technology & 2920 \\
\hline anti-p(S473)-AKT & Cell Signaling Technology & 9271 \\
\hline anti-PP2A-C & Cell Signaling Technology & 2038 \\
\hline anti-pS1390-gH2AX & Cell Signaling Technology & 9718 \\
\hline anti-GST & Cell Signaling Technology & 2624 \\
\hline anti- $\gamma$-H2AX & Millipore Sigma & $05-636$ \\
\hline anti-GAPDH & Millipore Sigma & MAB374 \\
\hline anti-Flag & Millipore Sigma & F1365 \\
\hline anti-53BP1 & Novus Biologicals & NP100-304 \\
\hline anti-Rad51 & Novus Biologicals & NB100-148 \\
\hline anti-PyMT & Novus Biologicals & NB100274955 \\
\hline anti- p(S4/S8)-RPA & Bethyl laboratories & A300-245A \\
\hline anti-ERK2 & Santa Cruz Biotechnology & sc-154 \\
\hline anti-pERK1/2 & Santa Cruz Biotechnology & sc-7383 \\
\hline anti-CRM1 & Santa Cruz Biotechnology & sc-74454 \\
\hline Alexa Fluor 594 goat anti-mouselgG & Life Technologies & R37121 \\
\hline Alexa Fluor 680 goat anti-rabbit IgG & Life Technologies & A-21076 \\
\hline Alexa Fluor 680 goat anti-mouse IgG & Life Technologies & A-21057 \\
\hline Dylight 800 anti-mouse IgG & Cell Signaling Technology & 5257 \\
\hline Dylight 800 anti-rabbit IgG & Cell Signaling Technology & 5151 \\
\hline Leptomycin B & Millipore Sigma & L2913 \\
\hline Camptothecin & Millipore Sigma & $7689-03-4$ \\
\hline KU-60019 & Selleckchem & S1570 \\
\hline
\end{tabular}

Immunoprecipitation. Flag-PR65 was immunoprecipitated from HEK293 cells stably expressing pMIG-Aalpha WT, S401A or S401D with anti-Flag M2 beads (Sigma Aldrich, Cat \#F2426) in lysis buffer with Halt ${ }^{\mathrm{TM}}$ protease and phosphatase inhibitor cocktail (ThermoFisher Scientific, Cat \#78440). YFP-ATM was immunoprecipitated by using the GFP-Trap Magnetic Agarose beads (Chromotek, Cat \#gtma-10). 
Photoactivation assay image processing. Temporal averages of the series were used for initial segmentation of cytoplasmic regions where mCherry fluorescence was stronger than in the corresponding irradiated nuclei. Segmentation thresholds were calculated by adding average and standard deviation (multiplied by 0.3 ) of fluorescence in the irradiated nuclear regions. The resulting binary masks were inverted and used to limit conditional dilation of the seeds (10x10 pixels) corresponding to the irradiated regions. Next the initial nuclear masks, created in this manner, were used construct 3pixel wide regions around them, at the distance of 4 pixels. Products of these regions and the result of the initial cytoplasm segmentation were used as cytoplasmic counterparts of the nuclear masks. These initial masks were updated tor every image in the post-irradiation series by conditional binary closing ( 2 iterations) of nuclear masks and according to construction of the cytoplasmic masks. Median intensity of the preirradiation images was used as background estimator. Average nuclear and cytoplasmic mCherry fluorescence intensities were corrected for background. The respective nucleus/cytoplasm ratios were averaged over cell populations.

Proximity Ligation assay. Proximity ligation assay was performed as per manufacture's recommendations (Duolink ${ }^{\circledR}$ using PLA ${ }^{\circledR}$ technology from Sigma Aldrich). PR65 KO MEFs expressing Flag-tagged WT PR65 were seeded on Lab-Tek (Naperville, IL) glass slides and treated with Leptomycin B (2 ng/ml) or not for 3 hours, irradiated with $10 \mathrm{~Gy}$ or not, or left untreated. Cells were fixed after 15 min followed by rabbit anti-PR65 and mouse anti-CRM1 antibodies to determine the extent of the PR65CRM1 interaction. Images were obtained by confocal microscopy. 


\section{Supplemental Figure Legends}

\section{Supplemental Figure 1. SV40 small-t and polyoma Middle-T inhibit PP2A and blocks radiation-induced AKT activation. (A) SV40 t (SVST) and Polyoma MT} (PyMT) antigens are known to replace the PP2A-B subunit and bind to the PP2A-A and C Core enzyme to inhibit PP2A activity and increase AKT phosphorylation (1) (B) Inhibition of ATM does not reduce PAKT (S473) levels in HEK293T cells but does in U87, U1242, and HEK293 cells. U87, U1242, HEK293, and 293T cells were treated with or without KU-60019 (3 $\mu \mathrm{M})$ for 1 hour. Whole cell extracts were separated on an SDSPAGE gel and analyzed by western blotting using anti-pAKT (S473) and anti-AKT antibodies. (C) U1242 and (D) U87 cells expressing PyMT antigen show diminished ATM-mediated inhibition of PAKT (S473) levels after radiation. U1242 and U87 cells were infected with pBabe-hygroPyMT vector or control vector pBabe-hygro. Infected cells were pre-treated with or without KU-60019 $(3 \mu \mathrm{M})$ for 1 hour followed by 5 Gy and whole cell extracts prepared at one hour post-IR were separated on an SDS-PAGE gel and analyzed by western blotting using anti-p-AKT and anti-AKT antibodies. (E) U1242 and U87 infected with PyMT virus are positive for virus integration. (F) Treatment of nuclear extract with okadaic acid increases pAKT levels. Nuclear extract was prepared from human glioma U87 cells and incubated with OA $(3,10,30$, or $100 \mathrm{nM})$ or not for 2 hours. Samples were then separated by SDS-PAGE and transferred to a membrane followed by sequential western blotting with anti-pAKT (S473) and AKT antibodies. Quantification of band intensity was done by densitometric scanning. 


\section{Supplemental Figure 2. ATM kinase phosphorylates PR65 in vitro. (A) HEK293} cells were transfected with YFP-ATM +/- mRuby2-PR65 constructs and sorted with only YFP-ATM/PR65 (S401A) cells surviving suggesting that ATM and PR65 S401A, but not WT or D, can be stably co-expressed in HEK293 cells whereas S401 phosphorylation or expression of S401D phosphor-mimetic is not tolerated with over-expressed ATM. FT; flow-through. (B) Whole cell extracts of HEK293 cells expressing YFP-ATM were immunoprecipitated with GFP-TRAP (ChromoTek). (C) In vitro kinase assay was performed by suspending YFP-ATM immunoprecipitate w/o co-expressed PR65 in buffer with $\left[\gamma^{-32} \mathrm{P}\right]$ ATP, $20 \mu \mathrm{M}$ unlabeled ATP, GST-substrates (GST-PR65FL or GSTp53 100$)$ with or without KU-60019 $(1 \mu \mathrm{M})$ for 1 hour. Samples were separated on a SDSPAGE gel and then analyzed by a Typhoon phosphoimager (PerkinElmer) (top panel). Phosphorylation of p53 and PR65 is reduced in the presence of KU-60019 (lane 3 and lane 6) relative to controls (lane 2 and 5), respectively. Coomassie Blue $(C B)$ - stained image of the gel showing GST-p53 and GST-PR65 proteins (bottom panel).

\section{Supplemental Figure 3. S401 mutant cells are more sensitive to camptothecin than wild type MEFs. WT, S401A and S401D MEFs were treated with CPT and analyzed by Cell titer-Glo $\AA$ assay at 96 hours to determine cell death. Error bars; mean \pm SEM $(n=3)$. CPT IC 50 is presented in the table.}

\section{Supplemental Figure 4. PCR and western blot analyses to verify floxing of endogenous PR65 sequences in MEFs stably transfected with DR-GFP. PCR} screening with primers $\mathrm{P} 1$ and $\mathrm{P} 3$ generated a 263-bp product for the WT allele and a 
417-bp product for the CKO allele. In cells where Cre was expressed, primer pair P3 and P2 generated a 587-bp product for the PR65 KO allele (top panel). Whole cell extracts of WT, S401A, and S401D MEFs with integrated DR-GFP infected with Ad-Cre or not were analyzed by western blotting with anti-PR65 antibody (bottom panel). Notice that the bottom PR65 band is missing after floxing indicating the loss of the endogenous allele. In addition, Flag-PR65 levels of WT, S401A, and S401 are very similar (compare with Fig. 1E).

\section{Supplemental Fig 5. Translocation between DSBs (CRISPR-Cas9) at Rosa26 (Chr}

6) and H3f3b (Chr 11). Sequences of Chr(11) breakpoint junction from WT (A), S401A (B) and S401D (C) cells. Reference sequence is highlighted at the top (blue). The remaining DNA sequences represent individual translocations recovered by PCR and subject to Sanger sequencing. Nucleotide insertions are marked in red. Nucleotide deletions are represented as gaps. Micro-homology is denoted by cyan highlighted nucleotides.

\section{Supplemental Figure 6. Putative PR65 NES in close proximity to S401. (A) PR65} has a putative nuclear export sequence (highlighted in red). (B) Model for PR65-CRM1 interaction via PR65-S401 and CRM1-K537. PR65-CRM1 co-crystal modeling showing a possible key interaction between PR65-S401 and CRM1- K537. PR65-CRM1 is colored in cyan whereas CRM1 is colored in silver. (C) PR65 S401 phosphorylation might expose a buried PR65 NES. A putative NES is located in the inter-repeat loop between HEAT domains 10-11 of PR65 and in close proximity to S401. Co-crystal 
structure of PP2A with PR65 structural $(A)$, catalytic $(C)$, and $(B)$ regulatory subunits with S401 and putative NES at L373 shown in yellow (left panel). Interaction domains between PR65 (A) and catalytic (C) subunits (right panel). The yellow portion of PR65 marks the domain directly interacting with the PP2A catalytic subunit (brown). PR65S401 is located at the rim whereas L373 is buried deeper at the interface between the PR65 and the PP2A catalytic subunit (left panel).

\section{Supplemental Figure 7. Expression of PR65-NES and S401A/NES and S401D/NES}

mutants. Transfection of HEK293 followed by western blotting of extracts shows approximately similar expression levels with anti-Flag and -PR65 antibodies. Notice the size difference between endogenous PR65 and much larger PAmCherry-PR65 proteins.

\section{Supplemental Figure 8. Hoechst 33258 pretreatment sensitizes cells to DNA}

damage. HEK293 cells were exposed to IR (5 Gy, 15 minutes) with or without Hoechst $33258(1,3$, or $10 \mu \mathrm{g} / \mathrm{ml}$; 3 hours $)$ treatment or treated with UV-A $\left(0.20 \mathrm{~J} / \mathrm{m}^{2} / \mathrm{s}\right.$ for 10 , 20 or 40 minutes) with or without Hoechst 33258 (1, 3 or $10 \mu \mathrm{g} / \mathrm{ml}$; 3 hours) treatment or treated with Hoechst 33258 alone (1, 3 or $10 \mu \mathrm{g} / \mathrm{ml}$; 3 hours). Cells were immunostained with anti-pKAP1 (S824) and counterstained with DAPI. Images were acquired at 63x power.

Supplemental Figure 9. PR65 nuclear - cytoplasmic shuttling. Nuclear and cytoplasmic fluorescence over time. HEK293 cells were transfected with pPAmCherryPR65 (WT, S401A and S401D) in a glass bottom dish. Forty-eight hours post- 
transfection sub-nuclear ROI (4 $\mathrm{m} \times 4 \mu \mathrm{m})$ were photo-activated by $405 \mathrm{~nm}$ laser and cells monitored over time by live cell imaging performed on Zeiss LSM 710 and images were acquired at $63 x$.

\section{Supplemental Figure 10. Tolerability of B subunit overexpression in PR65 MEFs.} PR65-WT, -S401A, and -S401D MEFs were transfected with pCEP-4HA-B56 alpha, B56 gamma3, -B56 gamma1, -B56 epsilon, -B56 delta, -B56 beta, or -B55 beta and selected for hygromycin $(200 \mu \mathrm{g} / \mathrm{ml})$ resistance. pCEP plasmids are maintained episomally as EBVori-EBNA plasmids in transfected MEFs (2).

\section{Supplemental Table 1. Statistics for AFM studies}

Four samples were amplified: wild-type (WT, $n=11,303$ measured strands), S401A ( $n=$ 6,530), S401D ( $n=12,160)$, and a synthetic fragment (Syn, $n=6,035$ ) of equivalent length and sequence as WT. Gaussian distribution of 3 populations for WT, S01D and Syn and five populations for S401A was analyzed. Analysis of traced length distributions was performed by fitting Gaussian mixture models then analyzing the Bayesian information criteria to determine the number of Gaussian distributions that produced the best fit. The $95 \%$ confidence interval for the mean of each fitted Gaussian was determined using a bootstrapping approach with $n=10,000$.

\section{Supplemental Table 2. Statistics for photoactivation experiment (Figure 9E). One} way ANOVA test was conducted for the nuclear to cytoplasmic intensity ratios in the time series studies for WT, S401A and S40D as well as NES, NES-S401A and NES- 
S401D groups. On Multiple comparison test, the differences between WT vs S401A, WT vs S401D, NES vs NES-S401A and NES vs NEs-S401D were highly significant. We also performed simple linear regression on the time series of the N/C intensities and found that the slopes of WT, S401A and S401d as well as NES, NES-S401A and NES S401D were significant different than each other. 


\section{References}

1. Campbell KS, Auger KR, Hemmings BA, Roberts TM, Pallas DC. Identification of regions in polyomavirus middle $T$ and small $t$ antigens important for association with protein phosphatase 2A. J Virol 1995;69(6):3721-8.

2. Einav Y, Shistik E, Shenfeld M, Simons AH, Melton DW, Canaani D. Replication and episomal maintenance of Epstein-Barr virus-based vectors in mouse embryonal fibroblasts enable synthetic lethality screens. Mol Cancer Ther 2003;2(11):1121-8. 


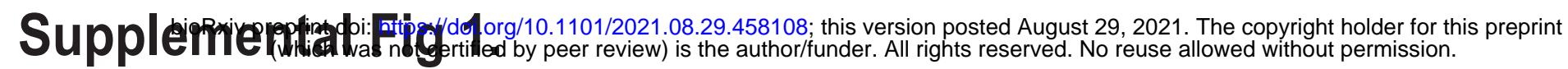

A
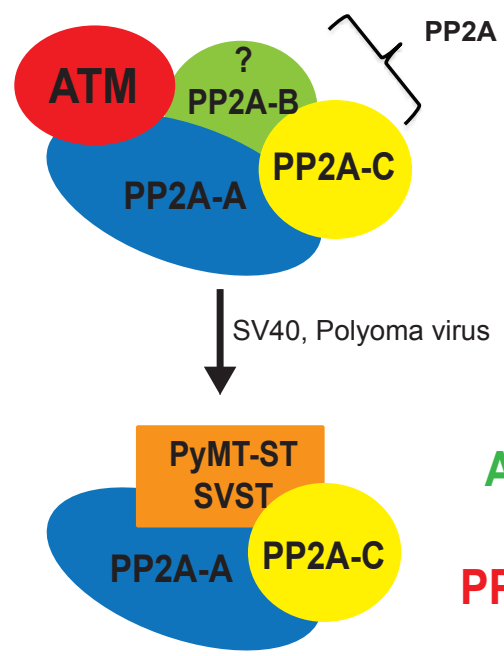

D

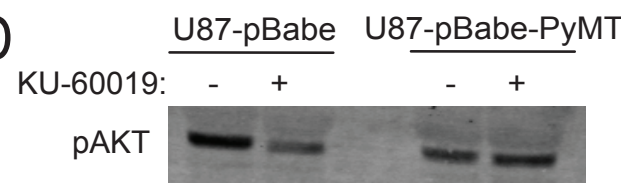

Fold: $\quad \begin{array}{llll}1 & 0.4 & 1 & 1.1\end{array}$

AKT

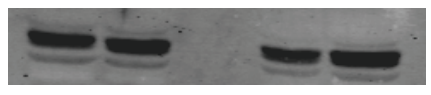

$E$

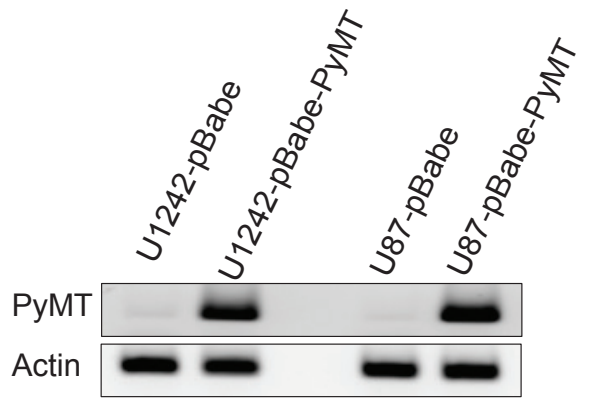

B

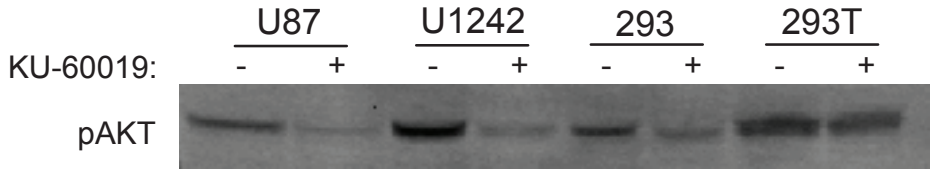

$\begin{array}{lllllllll}\text { Fold: } & 1 & 0.1 & 1 & 0.1 & 1 & 0.4 & 1 & 0.9\end{array}$

AKT
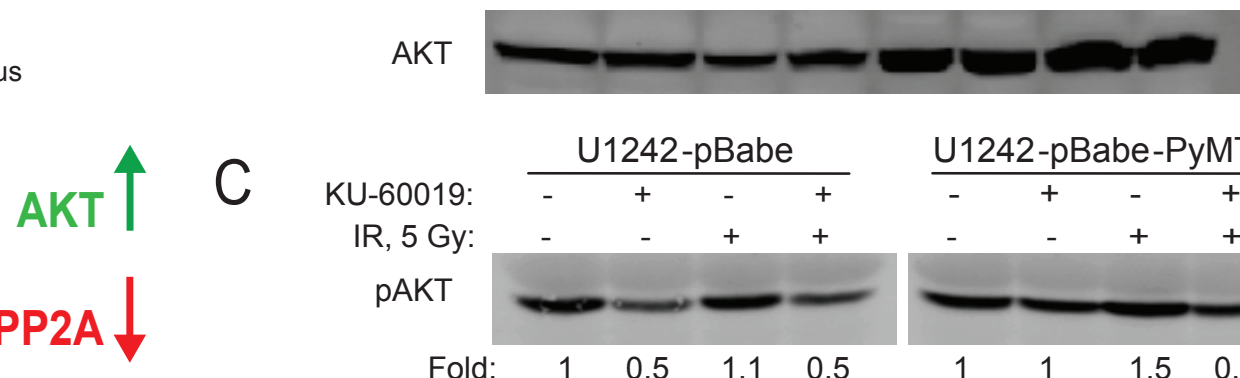

$\frac{\text { U1242-pBabe-PyMT }}{-\quad+\quad-\quad+}$

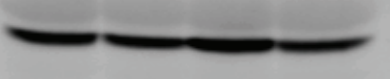

$\begin{array}{llll}1 & 1 & 1.5 & 0.9\end{array}$

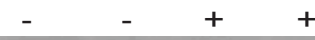

C

KU-60019:

IR, 5 Gy:

pAKT

Fold: AKT
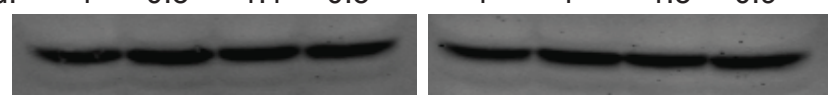

F
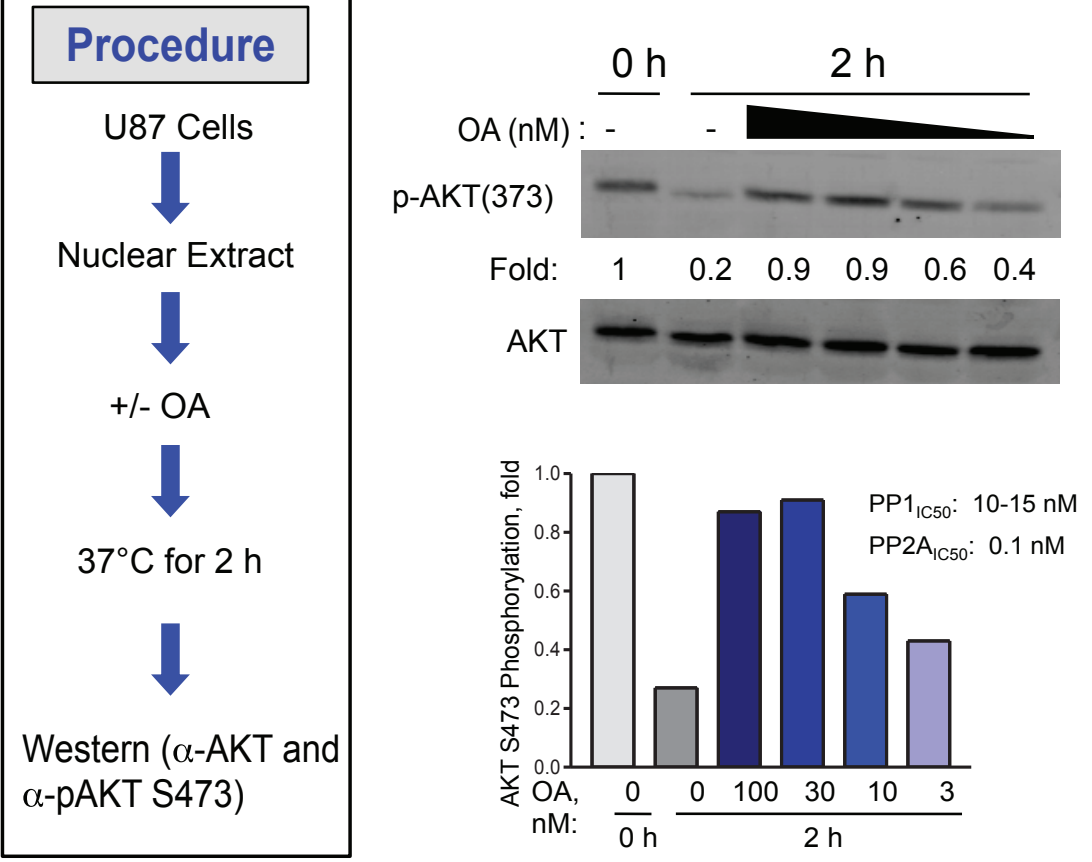


\section{IP-kinase assay}

A

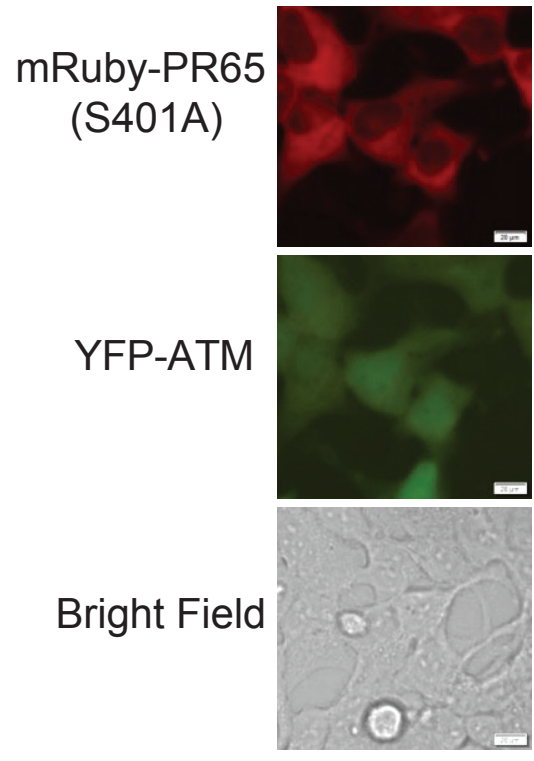

B IP-GFP TRAP

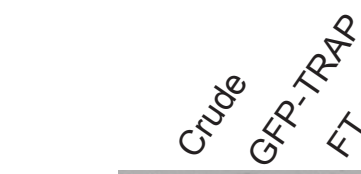

ATM

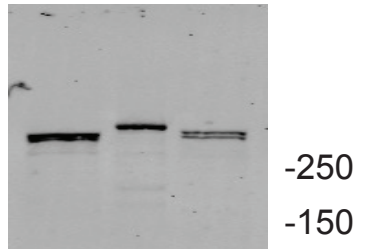

C

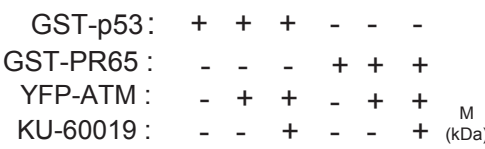

KU-60019 :

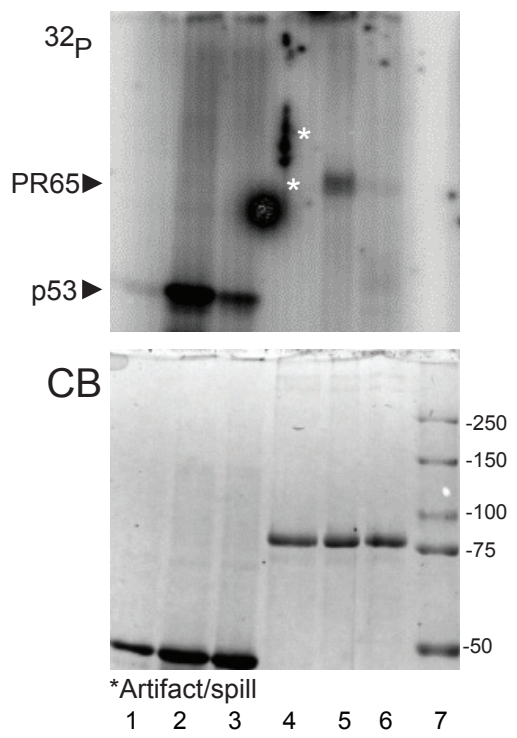




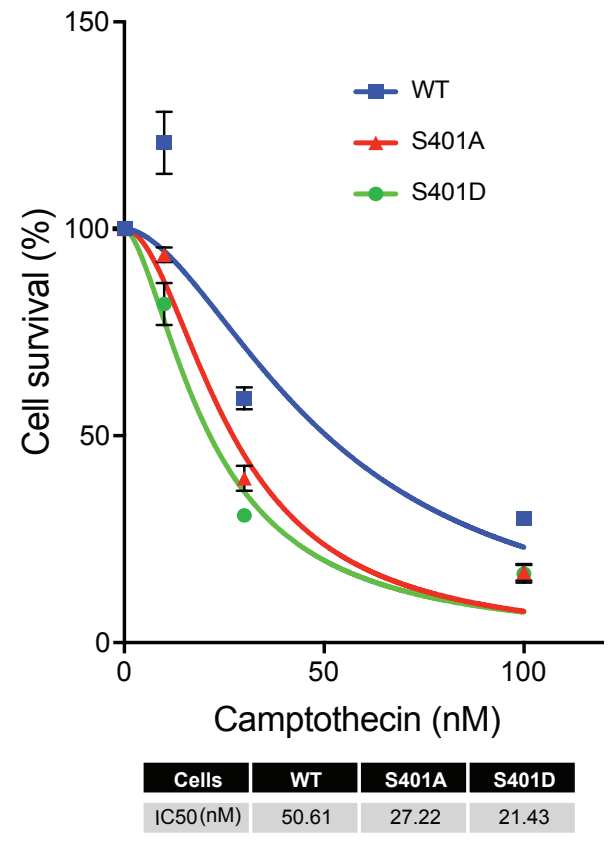




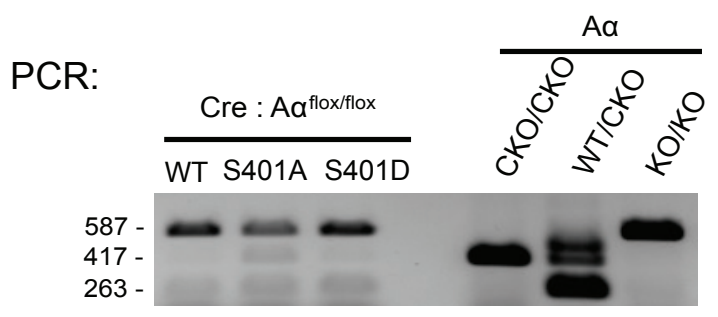

WB:

- Cre

+ Cre

$\overline{\text { WT S401A S401D }} \overline{\text { WT S401A S401D }}$

PR65

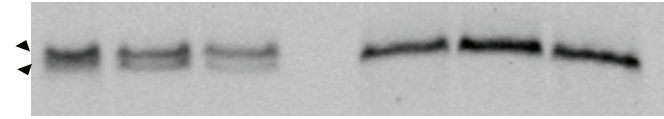




\section{MEFs expressing PR65 WT}

Chr-11

ATGCCATAAAAACCGCTTCAACTTAAGCTCTCTCCCCCCGTATCCGGCGAGCC

ATGCCATAAAAACCGCTTCAACTTAAGCTCTCTCCCCCC

ATGCCATAAAAACCGCTTCAACTTAAGCTCTCTCCCCCC CTGCCCTCCTCTCCGCTCCAGATTAGCCTCTCTCCCCCC ATGCCTTAAAAACCTCTTCAACTTAAGCTCTCTCCCCCC ATGCCATAAAAACCGCTTCAACTTAAGCTCTCTC ATGCCATAAAAACCGCTTCAACTTAAGCTCTCTCCCCCC ATGCCATAAAAACCGCTTCAACTTAAGCTCTCTCCCCCCG ATGCCATAAAAACCGCCTCAACTTAAGCTCTCTCCCCCC ATGCCATAAAAACCGCCTCAACTTAAGCTCTCTCCCCCC ATGCCATAAAAACCGCTTCAACTTAAGCTCTCTCCCCCC ATGCCATAAAAACCGCTTCAACTTAAGCTCTCTCCCCCC ATGCCATAAAAACCGCCTCAACTTAAGCTCTCTCCCCCC ATGCCATAAAAACCGCTTCAACTTAAGCTCTCTCCCCCCG ATGCCATAAAAACCGCTTCAACTTAAGCTCTCTCCCCCC ATGCCATAAAAACCGCTTCAACTTAAGCTCTCTCCCCCC ATGCCATAAAAACCGCCTCAACTTAAGCTCTCTCCCCCC ATGCCATAAAAACCGCCTCAACTTAAGCTCTCTCCCCCC ATGCCATAAAAACCGCCTCAACTTAAGCTCTCTCCCCCC ATGCCATAAAAACCGCTTCAACTTAAGCTCTCTCCCCCCG ATGCCATAAAAACCGCTTCAACTTA ATGCCATAAAAACCGCTTCAACTTAAGC TCTCTC ATGCCATAAAAACCGCTTCAACTTAAGCTCTCTCCCCCCG ATGCCATAAAAACCGCTTCAACTTAAGCTCTCTCCCCCCG

\section{Chr-6}

TCTAGAAAGACTGGAGTTGCAGATCACGAGGGAAGAGGGGGAAGGGATTCT TCTAGAAAGACTGGAGTTGCAGATCACGAGGGAAGAGGGGGAAGGGATTCT TCTAGAAAGAGTGGAGTTGGAGATCACGAGGGAAGAGGGGGAAGGGATTCT TCTAGAAAGACTGGAGTIGCAGATCACGAGGGAAGAGGGGGAAGGGATTCT TCTAGAATTACTGGAGTTGCGGATCTCGAGGGAAGAGGGGGAAGGGATTCT TCTATAAAGACTGGAGTTGCTGATCACGAGGGAANAGGGGGAAGGGTITCT GGAGTTGCAGATCACGAGGGAAGAGGGGGAAGGGATTCT GGAGTTGCAGATCACGAGGGAAGAGGGGGAAGGGATTCT TCTAGAAAGACTGGAGITGCAGATCACGAGGGAAGAGGGGGAAGGGATCT TCTAGAAAGACTGGAGTTGCAGATCACGAGGGAAGAGGGGGAAGGGATTCT TCTAGAAAGACTGGAGTTGCAGATCACGAGGGAAGAGGGGGAAGGGATTCT GTTGCAGATCACGAGGGAAGAGGGGGAGGGATTCT TCTAGAAAGACTGGAGTTGCAGATCACGAGGGAAGAGGGGGAAGGGATTCT TCTAGAAAGACTGGAGTTGCAGATCACGAGGGAAGAGGGGGAAGGGATTCT GTTGCAGATCACGAGGGAAGAGGGGGAGGGATTCT TCTAGAAAGACTGGAGTTGCAGATCACGAGGGAAGAGGGGGAGGGATTCT TCTAGAAAGACTGGAGTTGCAGATCACGAGGGAAGAGGGGGAAGGGATTCT TCTAGAAAGACTGGAGTIGCAGATCACGAGGGAAGAGGGGGAAGGGATTCT GTTGCAGATCACGAGGGAAGAGGGGGAAGGGATTCT

TCTAGAAAGACTGGAGTTGCAGATCACGAGGGAAGAGGGGGAAGGGATTCT GTTGCAGATCACGAGGGAAGAGGGGGAGGGATTCT GTTGCAGATCACGAGGGAAGAGGGGGAAGGGATTCT TCTAGAAAGACTGGAGTTGCAGATCACGAGGGAAGAGGGGGAAGGGATTCT TCTAGAAAGACTGGAGTTGCAGATCACGAGGGAAGAGGGGGAAGGGATTCT TCTAGAAAGACTGGAGTTGCAGATCACGAGGGAAGAGGGGGAAGGGATTCT

\title{
MEFs expressing PR65 S401A
}

\section{Chr-11}

ATGCCATAAAAACCGCTTCAACTTAAGCTCTCTCCCCCCGTATCCGGCGAGCC

ATGCCATAAAAACCGCTTCAACTTAAG ATGCCATAAAAACCGCTTCCACTTAAG

ATGCCATAAAAACCGCTTCAACTTAAGCTCTCTCCCCCCG ATGCCATAAAAACCGCCTCAACTTAAGCTCTCTCCCCCCG ATGCCATAAAAACCGCCTCAACTTAAGCTCTCTCCCCCCG ATGCCATAAAAACCGCTTCAACTTAAGC ATGCCATAAAAACCGCCTCAACTTAAGCTCTCTCCCCCCG ATGCCATAAAAACCGCCTCAACTTAAGCTCTCTCCCCCCG ATGCCATAAAAACCGCTTCAACTTAAGCTCTCTCCCCC ATGCCATAAAAACCGCTTCAACTTAAGCTCTCTCCCCCCG ATGCCATAAAAACCGCTTCAACTTAAGCTCTCTCCCCCCG ATGCCATAAAAACCGCTTCAACTTAAGCTCTCTCCCCCCG ATGCCATAAAAACCGCCTCAACTTAAGCTCTC ATGCCATAAAAACCGCCTCAACTTAAGCTCTC ATGCCATAAAAACCGCCTCAACTTAAGCTCTC ATGCCATAAAAACCGCCTCAACTTAAGCTCTC ATGCCATAAAAACCGCTTCAACTTAAGCTCTCTCCCCCC TCTTACAAAAAAAGTCACAAATTAAACCAAAGTATTITACAGAATTTACTACAAAATGCCATAAAAACCGCTTCA ATATTATAAACAATTTCTTACAAAAAAAGTCACAAATTAAACCAAAGTATTTTACAGAATTTACTACAAAATGCCATA

\section{Chr-6}

TCTAGAAAGACTGGAGTTGCAGATCACGAGGGAAGAGGGGGAAGGGATTCT

TCTAGAAAGACTGGAGCTGCAGATCACGAGGGAAGAGGGGGAAGGGATTCT IIIAGAAAGACTGGAGTIGCAGATCACGAGGGAAGAGGGGGAAGGGATTCT TCTAGAAAGACTGGAGTTGCAGATCACGAGGGAAGAGGGGGAAGGGATTCT TCTAGAAAGACTGGAGTTGCAGATCACGAGGGAAGAGGGGGAAGGGATTCT TCTAGAAAGACTGGAGTTGCAGATCACGAGGGAAGAGGGGGAAGGGATTCT TCTAGAAAGACTGGAGTIGCAGATCACGAGGGAAGAGGGGGAAGGGATTCT TCTAGAAAGACTGGAGTTGCAGATCACGAGGGAAGAGGGGGAAGGGATTCT TCTAGAAAGACTGGAGTTGCAGATCACGAGGGAAGAGGGGGAAGGGATTCT GAGTTGCAGATCACGAGGGAAGAGGGGGAAGGGATTCT TCTAGAAAGACTGGAGTTGCAGATCACGAGGGAAGAGGGGGAAGGGATTCT TCTAGAAAGACTGGAGTTGCAGATCACGAGGGAAGAGGGGGAAGGGATTCT TCTAGAAAGACTGGAGTTGCAGATCACGAGGGAAGAGGGGGAAGGGATTCT TCTAGAAAGACTGGAGTIGCAGATCACGAGGGAAGAGGGGGAAGGGATTCT TCTAGAAAGACTGGAGITGCAGATCACGAGGGAAGAGGGGGAAGGGATTCT TCTAGAAAGACTGGAGTTGCAGATCACGAGGGAAGAGGGGGAAGGGATTCT TCTAGAAAGACTGGAGITGCAGATCACGAGGGAAGAGGGGGAAGGGATTCT TCTAGAAAGACTGGAGTTGCAGATCACGAGGGAAGAGGGGGAAGGGATTCT ACTGGAGTTGCAGATCACGAGGGAAGAGGGGGAAGGGATTCT AAAGACTGGAGTTGCAGATCACGAGGGAAGAGGGGGAAGGGATTCT

\section{MEFs expressing PR65 S401D}

\author{
Chr-11
}

Chr-6

ATGCCATAAAAACCGCTTCAACTTAAGCTCTCTCCCCCCGTATCCGGCGAGCC

TCTAGAAAGACTGGAGTIGCAGATCACGAGGGAGAGGGGGAGGGATTCT

ATGCCATAAAAACCGCCTCAACTTAAGCTCTCTCCCCACACACCAGGTTAGCCTTTAAGCCTGCCCAGAAGACTCCCGCCCATCTAGAAAGACTGGAGTTGCAGATCACGAGGGAAGAGGGGGAAGGGATTCT ATGCCATAAAAACCGCCTCAACTTAAGCTCTCTCCCCACACACCAGGTTAGCCTITAAGCCTGCCCAGAAGACTCCCGCCCATCTAGAAAGACTGGAGTTGCAGATCACGAGGGAAGAGGGGGAAGGGATTCT ATGCCATAAAAACCGCCTCAACTTAAGCTCTCTCCCCACACACCAGGTTAGCCTTTAAGCCTGCCCAGAAGACTCCCGCCCATCTAGAAAGACTGGAGTTGCAGATCACGAGGGAAGAGGGGGAAGGGATTCT ATGCCATAAAAACCGCTTCAACTTAAGCTCTCTCCCCCC ATGCGATAAAAACCGCTTCAACTTAAGCTCTCTCCCCCC ATGCCATAAAAACCGCTTCAACTTAAGCTCTCTCCCCCC

c TCTTACAAAAAAAGTCACAAATTAAACCAAAGTATITTACAGAATTTA ATGCCATAAAAACCGCTTCAA ATGCCATAAAAACCGCTTCAACTTAAGCTCTCTCCCCCCG ATGCCATAAAANCCGCTTCANCTTAAGCTCTCTCCCCCC ATGCCATAAAAACCGCTTCAACTTAAGCTCTCTCCCCCC ATGCGCTAAAAACCGCTTCAACTITTACTCTCTCCCCCC ATGCCATAAAAACCGCTTCAACTTAAGCTCTCTCCCCCCG ATGCCATAAAAACCGCTTCAACTTAAGCTCTCTCCCCCCG ATGCCATAAAAACCGTTTCAACTTAAGCTCTCTCCCCCC ATGCCATAAAAACCGCCTCAACTTAAGCTC ATGCCATAAAAACCGCTTCAACTTAAGCTCTCTCCCCCC ATGCCATAAAAACCGCTTCAACTTAAGCTCTCTCCCCCC ATGCCATAAAAACCGCTTCAACTTAAGCTCTCTCCCCCCG ATGCCATAAAAACCGCTTCAACTTAAGCTCTCTCCCCCCG ATGCCATAAAAACCGCTTCAA
TCTAGAAAGACTGGAATTGCAGATCACGAGGGAAGAGGGGGAAGGGATTCT TCTAGAAAGACTGGAGTIGCAGATCACAAGGGAAGAGGGGGAAGGGATTT TCTAGAAAGACTGGAGTTGCAGATCACGAGGGAAGAGGGGGAAGGGATTCT TCTAGAAAGACTGGAGTIGCAGATCACGAGGGAAGAGGGGGAAGGGATTCT CTAGAAAGACTGGAGTTGCAGATCACGAGGGAAGAGGGGGAAGGGATTCT CTAGAAAGACTGGAGTTGCAGATCACGAGGGAAGAGGGGGAAGGGATTCT

TCTAGAAAGACTGGAGTTGCAGATCACGAGGGAAGAGGGGGAAGGGATTCT AGACTGGAGTTGCAGATCACGAGGGAAGAGGGGGAAGGGATTCT

CTAGAAAGACTGGAGTTGCAGATCACGAGGGAAGAGGGGGAAGGGATTCT GACTGGAGTTACNGACTCGCNGATCGAGGGGGAAGGGGTTCT

TCTAGAAAGACTGGAGITGCAGATCACGAGGGAAGAGGGGGAAGGGATTCT

TCTAGAAAGACTGGAGTTGCAGATCACGAGGGAAGAGGGGGAAGGGATTCT AGACTGGAGTTGCAGATCACGAGGGAAGAGGGGGAAGGGATTCT TTGCAGATCACGAGGGAAGAGGGGGAAGGGATTCT AGACTGGAGTTGCAGATCACGAGGGAAGAGGGGGAAGGGATTCT AGACTGGAGTTGCAGATCACGAGGGAAGAGGGGGAAGGGATTCT TCTAGAAAGACTGGAGTTGCAGATCACGAGGGAAGAGGGGGAAGGGATTCT TCTAGAAAGACTGGAGTTGCAGATCACGAGGGAAGAGGGGGAAGGGATTCT

CTAGAAAGACTGGAGTTGCAGATCACGAGGGAAGAGGGGGAAGGGATTCT AAAGACTGGAGTTGCAGATCACGAGGGAAGAGGGGGAAGGGATTCT 
NES : LXXXLXxLXL

1 MAAADGDDSL YPIAVLIDEL RNEDVQLRLN SIKKLSTIAL ALGVERTRSE

51 LLPFLTDTIY DEDEVLLALA EQLGTFTTLV GGPEYVHCLL PPLESLATVE

101 ETVVRDKAVE SLRAISHEHS PSDLEAHFVP LVKRLAGGDW FTSRTSACG|

151 FSVCYPRVSS AVKAELRQYF RNLCSDDTPM VRRAAASKLG EFAKVLELD|

201 VKSEIIPMFS NLASDEQDSV RLLAVEACVN IAQLLPQEDL EALVMPTLRQ

251 AAEDKSWRVR YMVADKFTELQKAVGPEITKTDLVPAFQNL MKDCEAEVRA

301 AASHKVKEFC ENLSADCREN VIMTQILPCI KELVSDANQH VKSALASVIM

351 GLSPILGKDNTIEHLLPLFLAQLKDECPEV RLNIISNLDC VNEVIGIRQL

401 SQSLLPAIVE LAEDAKWRVR LAIIEYMPLL AGQLGVEFFD EKLNSLCMAW

451 LVDHVYAIRE AATSNLKKLV EKFGKEWAHA TIIPKVLAMS GDPNYLHRMT

501 TLFCINVLSE VCGQDITTKH MLPTVLRMAG DPVANVRFNV AKSLQKIGPI

551 LDNSTLQSEV KPILEKLTQD QDVDVKYFAQ EALTVLSLA

B

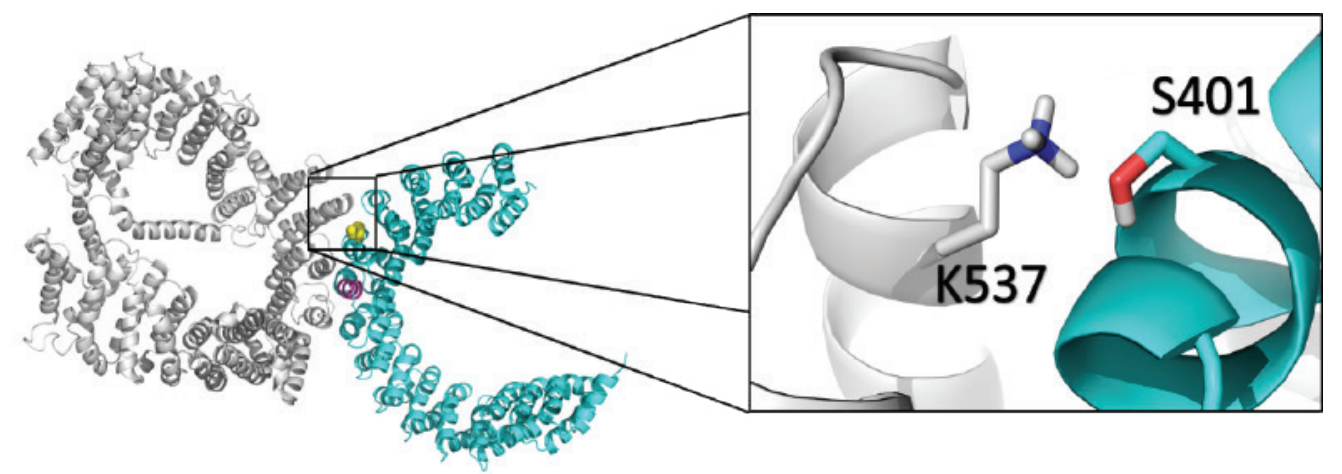

C

PR65: - $L_{365}$ LPLFLAQL $_{373}$ -

NES consensus: - L-X-X-LF-XX-LSV -

A: Structural (15 HEAT repeats)

B: Regulatory

C: Catalytic subunit
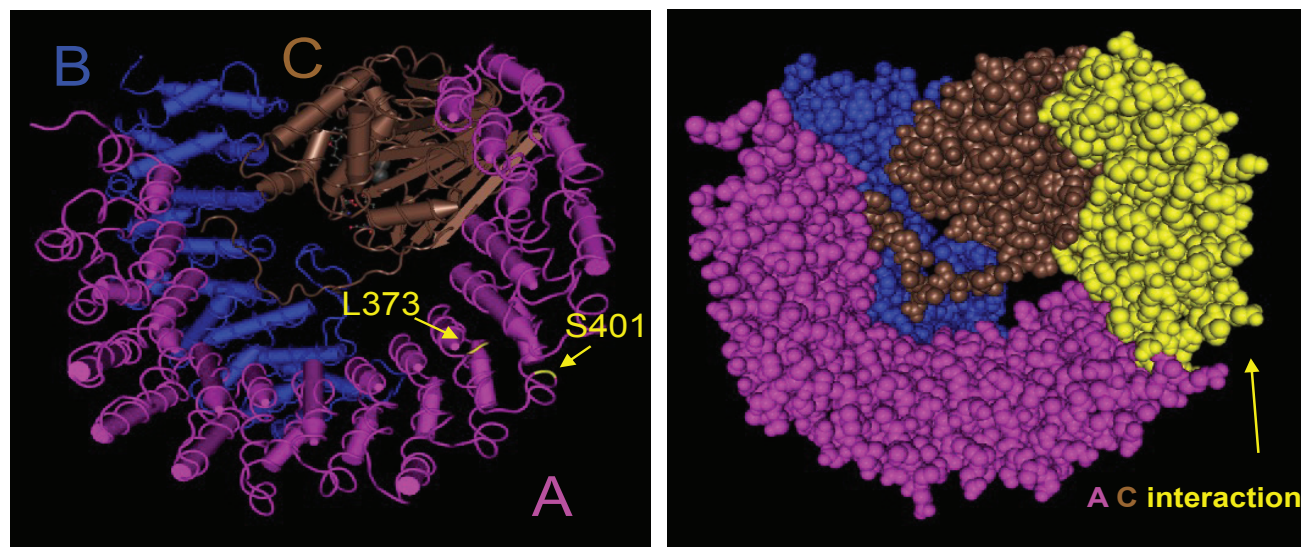


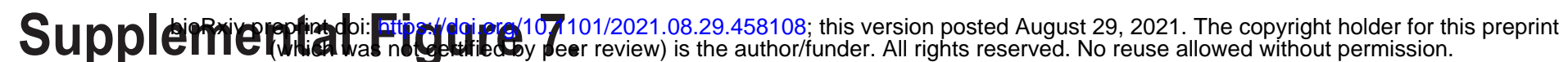

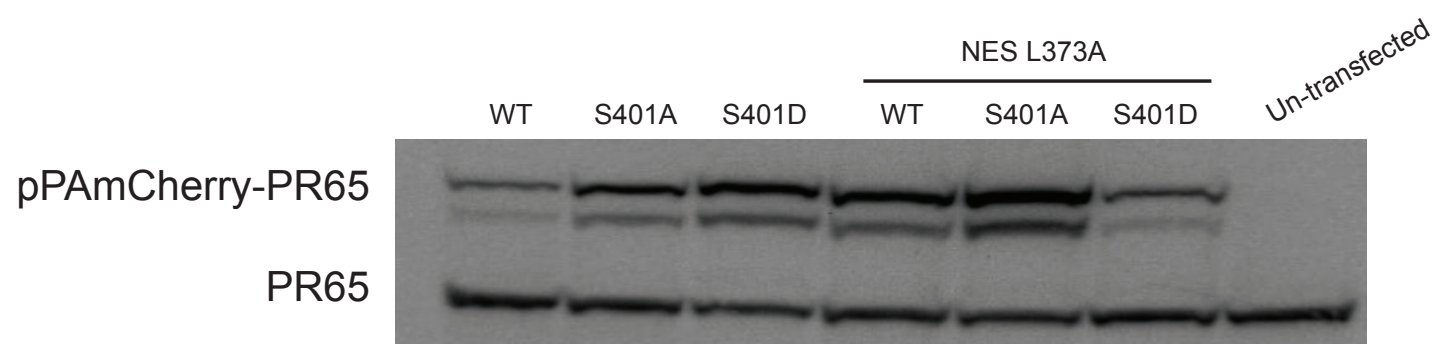




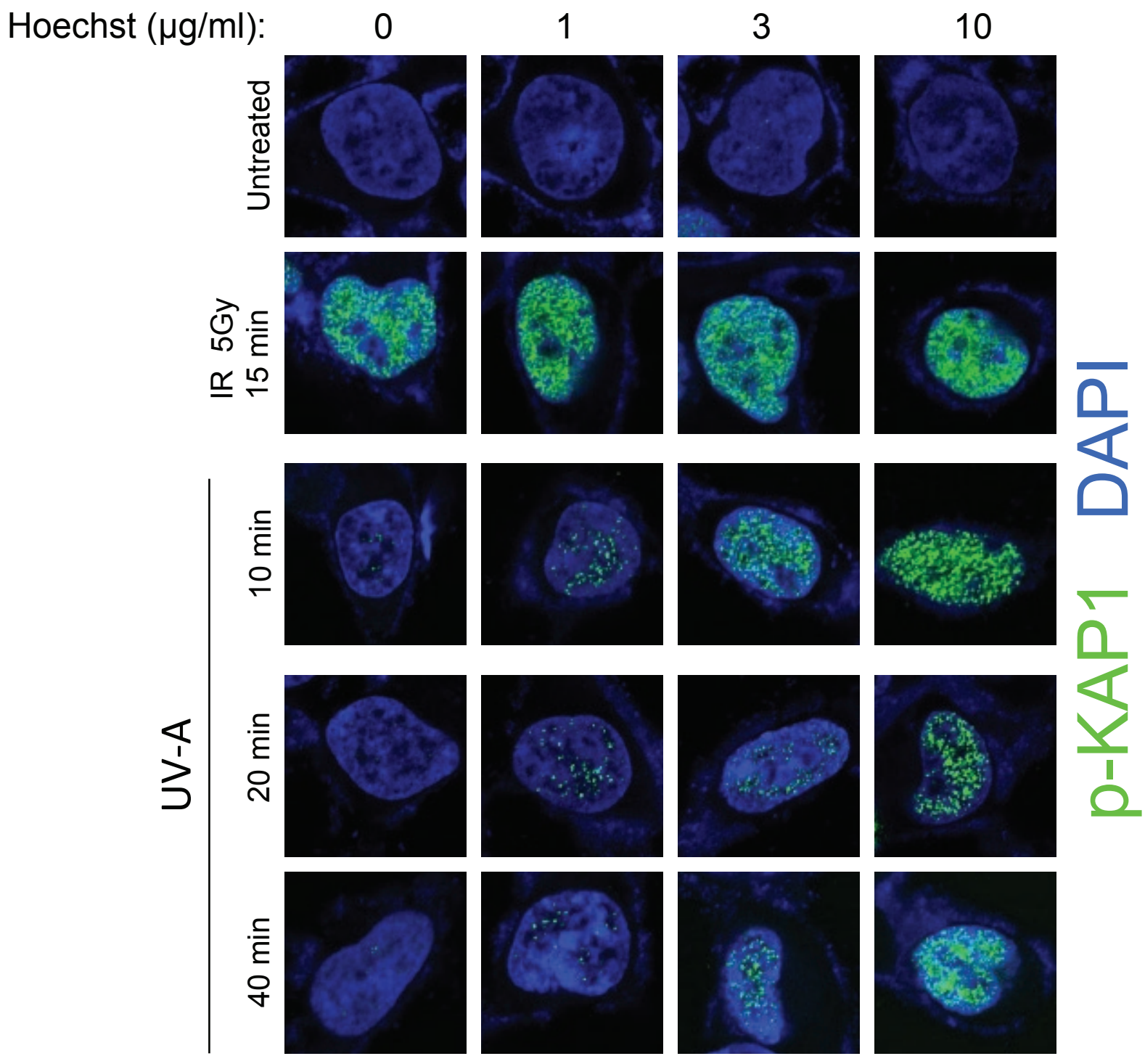




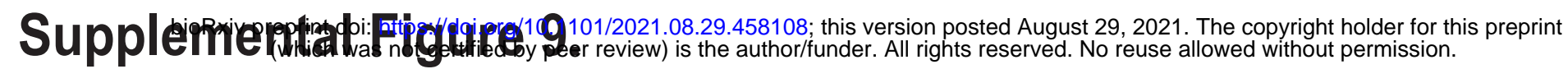

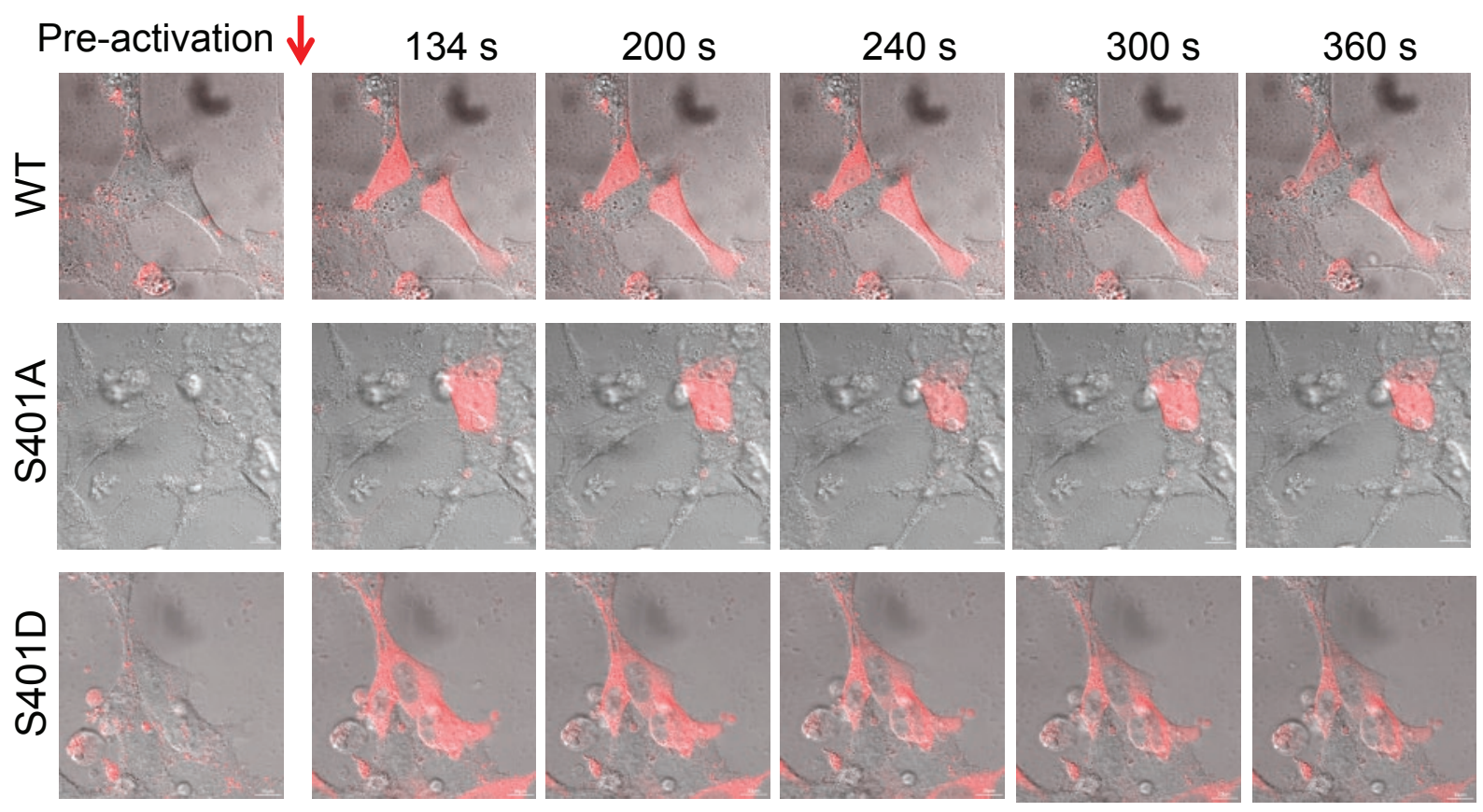


A
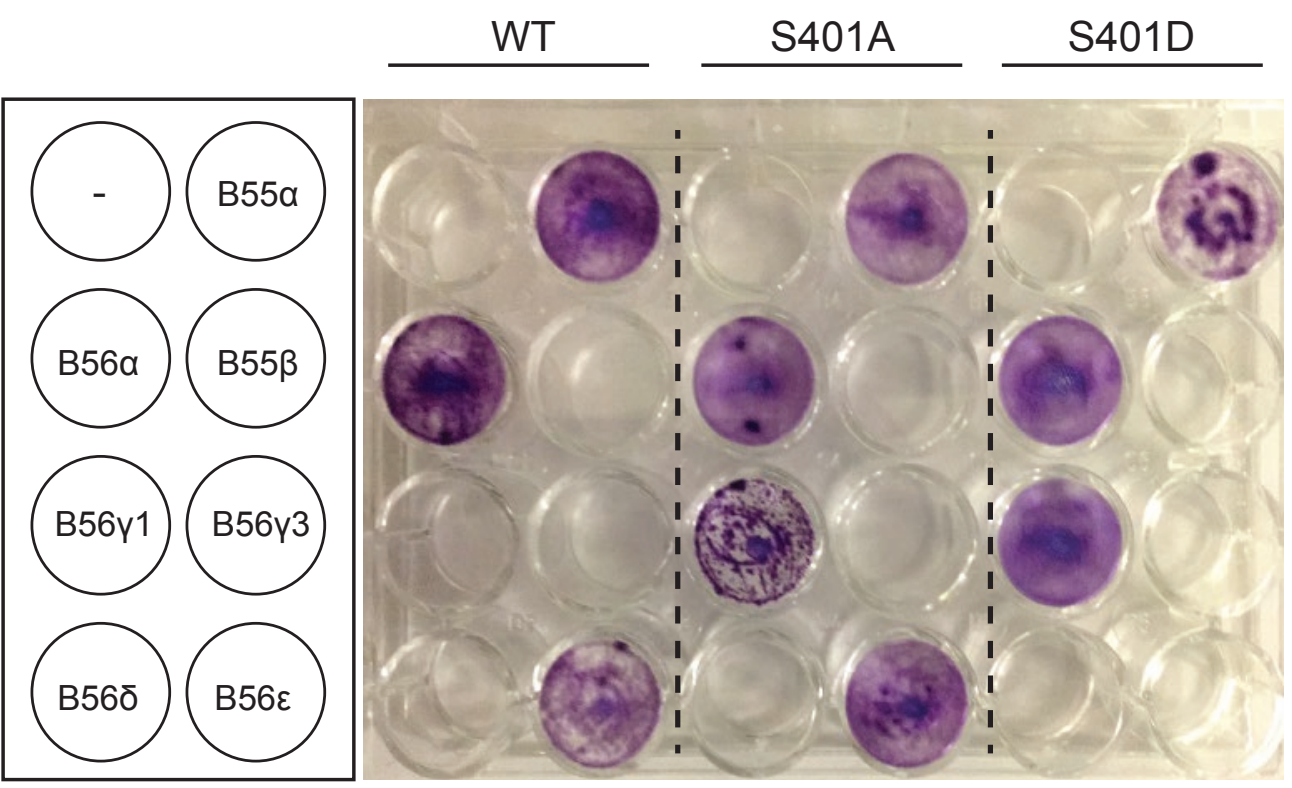

B

\begin{tabular}{|c|c|c|c|}
\hline B-subunit & WT & S401A & S401D \\
\hline B55 $\alpha / P P P 2 R 2 A$ & + & + & + \\
\hline B56 $\alpha / P P P 2 R 5 A$ & + & + & + \\
\hline B56 $/$ PPP2R5B & - & - & - \\
\hline B56 $\gamma 1 / P P P 2 R 5 C$ & - & + & + \\
\hline В56 $\gamma 3 /$ & - & - & - \\
\hline B568/PPP2R5D & - & - & - \\
\hline B56ع/PPP2R5E & + & + & - \\
\hline
\end{tabular}


Supplemental Table 1.

WT

\begin{tabular}{|l|r|r|r|}
\hline Row & \multicolumn{1}{|c|}{ gaussian1 } & \multicolumn{1}{l|}{ gaussian2 } & \multicolumn{1}{l|}{ aussian3 } \\
\hline $\mathrm{mu}$ & 112.6713334 & 47.99697261 & 172.1115854 \\
\hline $\mathrm{mu}$ [Cl_lower & 112.1608046 & 47.03594875 & 159.9828084 \\
\hline $\mathrm{mu}$ Cl_upper & 113.1693958 & 48.97239987 & 186.0252576 \\
\hline sigma & 20.57601541 & 13.99117091 & 94.11453542 \\
\hline sigma_Cl_lower & 20.13991044 & 13.10672902 & 85.39626477 \\
\hline sigma_Cl_upper & 21.02688002 & 14.89745807 & 102.5372019 \\
\hline proportion & 0.8072826 & 0.151702945 & 0.041014455 \\
\hline proportion_Cl_lower & 0.796783762 & 0.142667002 & 0.034361264 \\
\hline proportion_Cl_upper & 0.817558008 & 0.16064956 & 0.047994046 \\
\hline
\end{tabular}

\section{S401A}

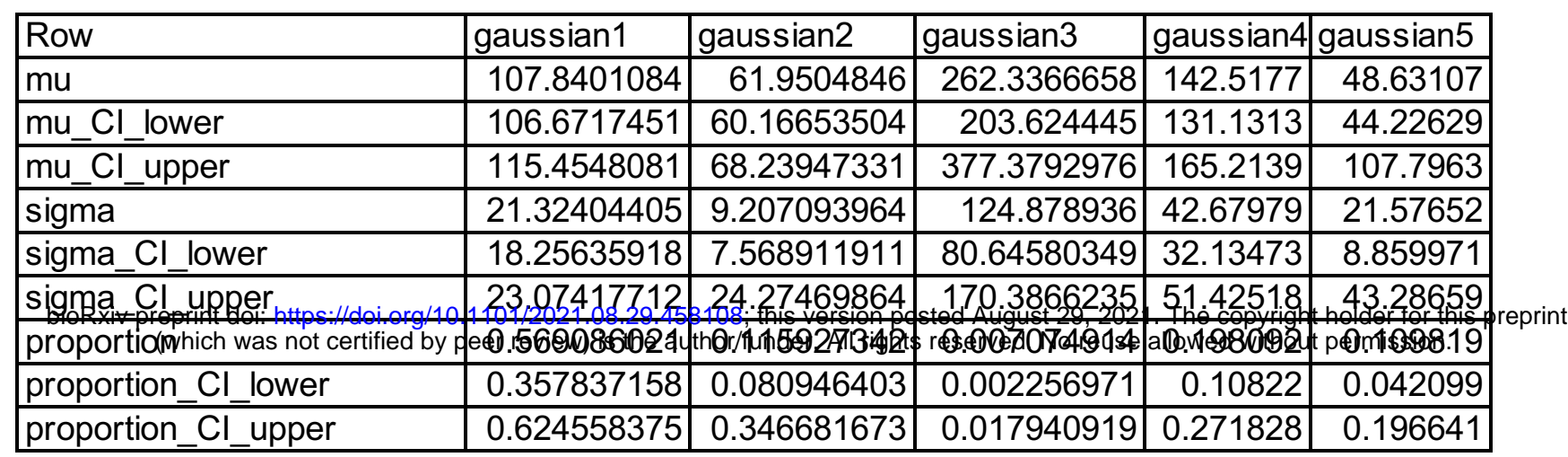

\section{S401D}

\begin{tabular}{|l|l|l|l|}
\hline Row & gaussian1 & gaussian2 & gaussian3 \\
\hline $\mathrm{mu}$ & 106.0315687 & 46.76675558 & 174.8895841 \\
\hline $\mathrm{mu} C \mathrm{Cl}$ _lower & 105.4597517 & 46.39652219 & 165.5553866 \\
\hline $\mathrm{mu} \mathrm{Cl}$ _upper & 106.6041131 & 47.13127314 & 185.3803763 \\
\hline sigma & 20.54104028 & 9.570053569 & 93.10054925 \\
\hline sigma_Cl_lower & 19.98778326 & 9.266241556 & 87.48585699 \\
\hline sigma_Cl_upper & 21.09092988 & 9.868076039 & 98.51755827 \\
\hline proportion & 0.607900591 & 0.335846752 & 0.056252658 \\
\hline proportion_Cl_lower & 0.597047248 & 0.326093943 & 0.049496932 \\
\hline proportion_Cl_upper & 0.619078528 & 0.345248898 & 0.063379692 \\
\hline
\end{tabular}

\section{Syn}

\begin{tabular}{|l|r|l|r|}
\hline Row & \multicolumn{1}{|l|}{ gaussian1 } & gaussian2 & \multicolumn{1}{|c|}{ gaussian3 } \\
\hline mu & 119.0488805 & 44.52886136 & 171.5183648 \\
\hline mu_Cl_lower & 118.570992 & 41.67076681 & 161.6553723 \\
\hline mu_Cl_upper & 119.597509 & 47.40926267 & 235.400503 \\
\hline sigma & 18.32874438 & 20.95980558 & 91.48017913 \\
\hline sigma_Cl_lower & 17.97499435 & 18.70377407 & 27.44910405 \\
\hline sigma_Cl_upper & 18.77843553 & 23.45894998 & 129.842548 \\
\hline proportion & 0.925191784 & 0.055257503 & 0.019550713 \\
\hline proportion_Cl_lower & 0.91788522 & 0.049143018 & 0.007864581 \\
\hline proportion_Cl_upper & 0.937139109 & 0.064220123 & 0.02474155 \\
\hline
\end{tabular}


Supplemental Table 2.

One-way ANOVA results

\begin{tabular}{|c|c|c|}
\hline Table analysed & WT, S401A and S401D & NES, NES-S401A and NES S401D \\
\hline Repeated measures ANOVA summary & & \\
\hline Assume sphericity? & No & No \\
\hline $\mathrm{F}$ & 12255 & 159153 \\
\hline Pvalue & $<0.0001$ & $<0.0001$ \\
\hline Pvalue summary & 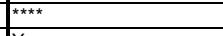 & \\
\hline Statistically significant $(P<0.05)$ ? & Yes & Yes \\
\hline Geisser-Greenhouse's epsilon & 0.5335 & 0.5574 \\
\hline R squared & 0.9227 & 0.9936 \\
\hline
\end{tabular}

\begin{tabular}{ll} 
Geisser-Gregh \\
R squared \\
\hline
\end{tabular}

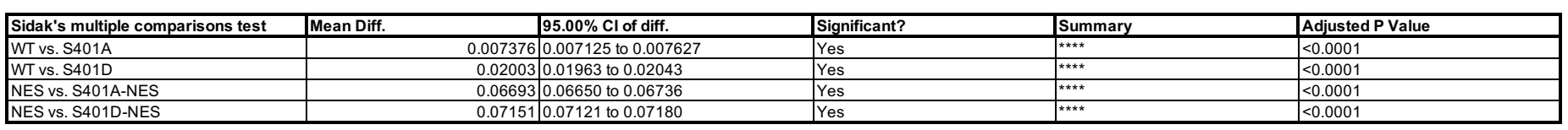

\begin{tabular}{|l|l|}
\hline NES vs. S401A-NES & 0.06930 .06650 to 0.06736 \\
\hline NES vs. S401D-NES & 0.071510 .07121 to 0.07180 \\
\hline
\end{tabular}

\begin{tabular}{|c|c|c|c|c|c|c|c|c|}
\hline Test details & Mean 1 & Mean 2 & Mean Diff. & SE of diff. & n1 & n2 & $t$ & DF \\
\hline WT vs. S401A & 0.03816 & 0.03079 & 0.007376 & 0.000112 & 1027 & 1027 & 65.83 & 1026 \\
\hline \begin{tabular}{|l|} 
WT vs. S401D \\
\end{tabular} & 0.03816 & 0.01813 & 0.02003 & 0.0001787 & 1027 & 1027 & 112.1 & 1026 \\
\hline NES vs. S401A-NES & 0.09468 & 0.02774 & 0.06693 & 0.000192 & 1027 & 1027 & 348.7 & 1026 \\
\hline NES vs. S401D-NES & 0.09468 & 0.02317 & 0.07151 & 0.000132 & 1027 & 1027 & 541.6 & 102 \\
\hline
\end{tabular}

\begin{tabular}{|c|c|c|c|c|c|c|}
\hline & WT & S401A & S401D & NES & S401A-NES & S401D-NES \\
\hline Slope & -0.00006907 & -0.00002349 & 0.000007893 & -0.0000744 & -0.00001349 & -0.00004356 \\
\hline S-iope & $\frac{-0.0000901}{0.04652}$ & $\begin{array}{r}-0.0002549 \\
0.03363\end{array}$ & $\begin{array}{r}0.01717 \\
\end{array}$ & $\begin{aligned}-0.000074 \\
\end{aligned}$ & $\frac{-0.00002937}{0.02937}$ & $\frac{-0.0004550}{0.02844}$ \\
\hline $\begin{array}{l}\text { Y-intercet } \\
\text { X-intercept }\end{array}$ & 0.0465 .5 & $\begin{array}{c}0.03303 \\
1431 \\
\end{array}$ & -2176 & $\begin{aligned} 0.1351 \\
1394 \\
\end{aligned}$ & $\frac{0.02931}{2178}$ & $\frac{0.02844}{652.9}$ \\
\hline 1/slope & \begin{tabular}{l|l|}
-14478 \\
\end{tabular} & -42564 & 126702 & -13441 & -74145 & -22958 \\
\hline Std. Error & & -5 & & -5 & -5 & \\
\hline $\begin{array}{l}\text { Slope } \\
\text { Yoptercept }\end{array}$ & \begin{tabular}{|c|}
$8.245 E-07$ \\
0.0001153 \\
\end{tabular} & $\begin{array}{r}0.000000587 \\
0.00008206\end{array}$ & $\begin{array}{r}3.543 \mathrm{E}-07 \\
00004952\end{array}$ & $\begin{array}{r}0.000001373 \\
0.000192\end{array}$ & $\begin{array}{l}6.394 \mathrm{E}-07 \\
00000938\end{array}$ & $\begin{array}{l}6.069 \mathrm{E}-07 \\
0000284\end{array}$ \\
\hline Y-intercept & 0.0001153 & & 0.00004952 & 0.000192 & & \\
\hline $95 \%$ Confidence Intervals & & & & & & \\
\hline Slope & & $-2.464 \mathrm{e}-005$ to $-2.234 \mathrm{e}-005$ & $7.198 \mathrm{e}-006$ to $8.587 \mathrm{e}-006$ & $-7.709 \mathrm{e}-005$ to $-7.171 \mathrm{e}-005$ & $-1.474 \mathrm{e}-005$ to $-1.223 \mathrm{e}-005$ & $-4.475 \mathrm{e}-005$ to $-4.237 \mathrm{e}-005$ \\
\hline X-intercept & 660.8 to 686.9 & 1370 to 1499 & -2398 to -1990 & 1349 to 1441 & 2003 to 2389 & 638.6 to 667.9 \\
\hline 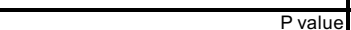 & $<0.0001$ & $<0.0001$ & $<0.0001$ & $<0.0001$ & $<0.0001$ & $<00001$ \\
\hline
\end{tabular}

